Prepared in cooperation with the U.S. Army Corps of Engineers, Nashville District

\title{
Estimated Use of Water in the Cumberland River Watershed in 2010 and Projections of Public-Supply Water Use to 2040
}
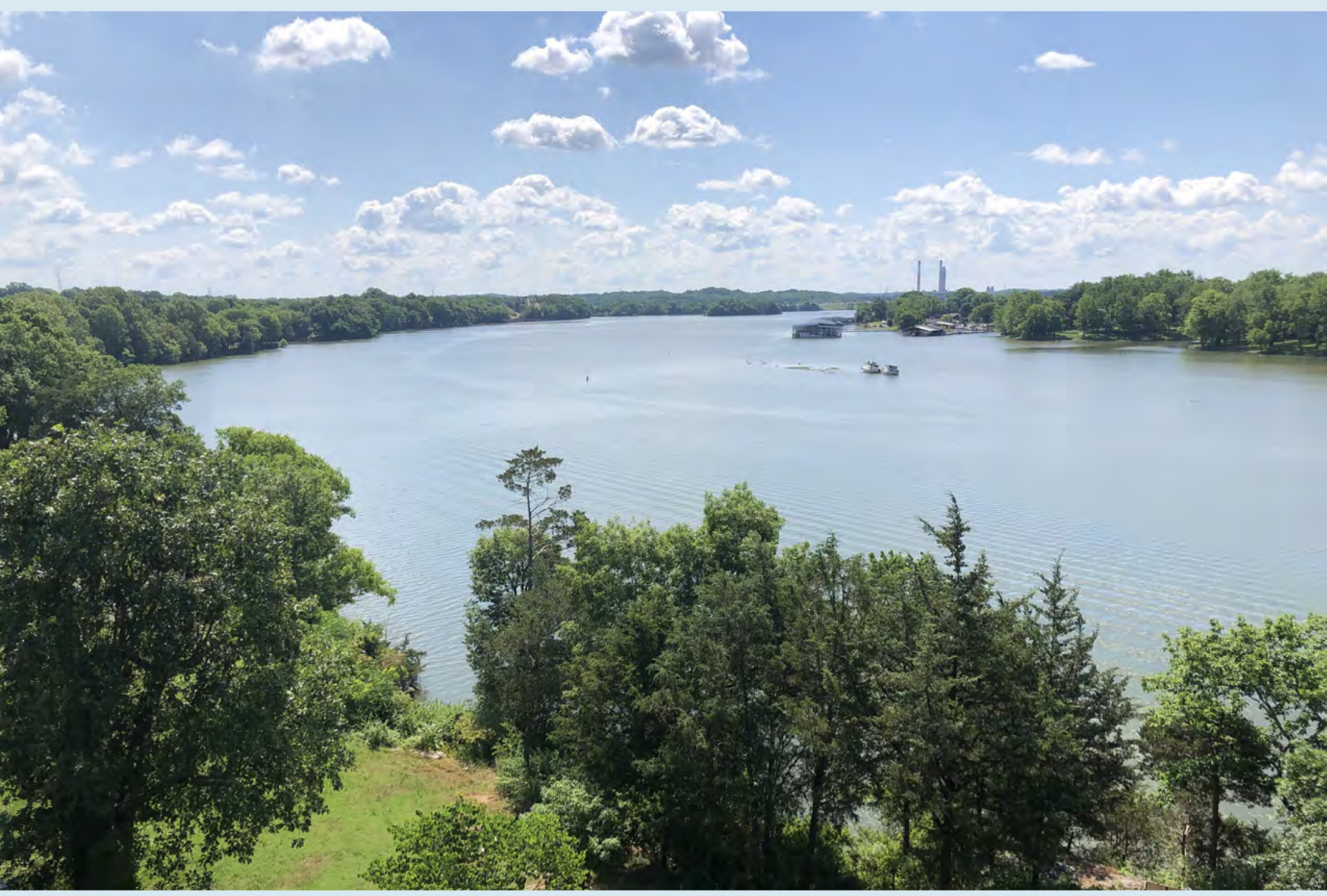

Scientific Investigations Report 2018-5130 
Cover. Cumberland River/Old Hickory Lake, Gallatin, Tennessee, May 31, 2019. 


\section{Estimated Use of Water in the Cumberland River Watershed in 2010 and Projections of Public-Supply Water Use to 2040}

By John A. Robinson

Prepared in cooperation with the U.S. Army Corps of Engineers, Nashville District

Scientific Investigations Report 2018-5130 


\title{
U.S. Department of the Interior \\ DAVID BERNHARDT, Secretary
}

\author{
U.S. Geological Survey \\ James F. Reilly II, Director
}

U.S. Geological Survey, Reston, Virginia: 2019

For more information on the USGS - the Federal source for science about the Earth, its natural and living resources, natural hazards, and the environment-visit https://www.usgs.gov or call 1-888-ASK-USGS.

For an overview of USGS information products, including maps, imagery, and publications,

visit https://store.usgs.gov.

Any use of trade, firm, or product names is for descriptive purposes only and does not imply endorsement by the U.S. Government.

Although this information product, for the most part, is in the public domain, it also may contain copyrighted materials as noted in the text. Permission to reproduce copyrighted items must be secured from the copyright owner.

Suggested citation:

Robinson, J.A., 2019, Estimated use of water in the Cumberland River watershed in 2010 and projections of publicsupply water use to 2040: U.S. Geological Survey Scientific Investigations Report 2018-5130, 62 p., https://doi. org/10.3133/sir20185130.

ISSN 2328-0328 (online) 


\section{Acknowledgments}

The author thanks Benjamin L. Rohrbach and Parvathi Gaddipati of the U.S. Army Corps of Engineers for their guidance in developing this report, for their technical reviews, and for sharing their knowledge about the reservoir catchment areas. Personnel from many State and local agencies in Tennessee and Kentucky are acknowledged for their assistance with the compilation of data for 2010.

Many employees of the U.S. Geological Survey are thanked for their contributions to this report. W. Scott Gain assisted in the development of the methods for water-use projections. Michael W. Bradley helped with the collection and compilation of the irrigation and industrial water-use data. David E. Ladd provided geographic information system support, James E. Banton helped with the figures, and Lori R. Weir assisted with the water system surveys. 


\section{Contents}

Acknowledgments .........................................................................................................................ii

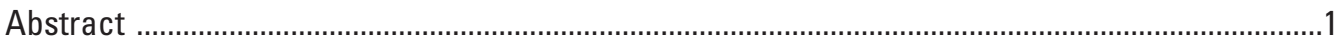

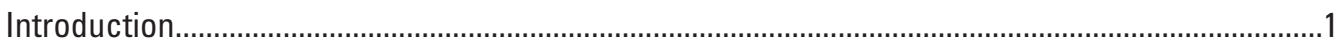

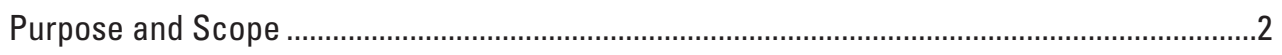

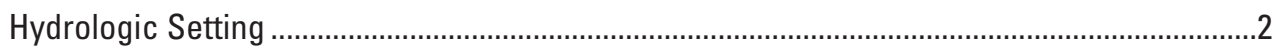

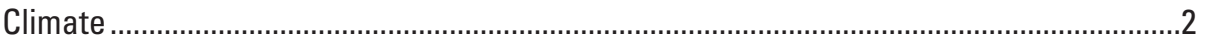

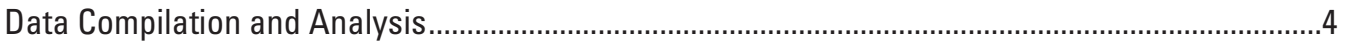

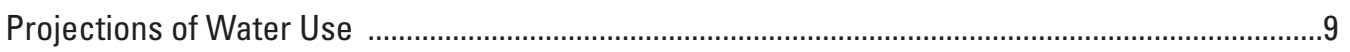

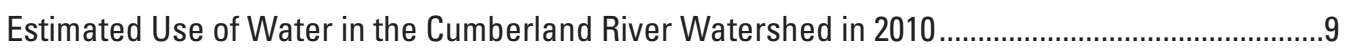

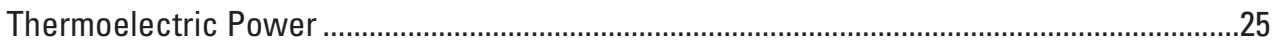

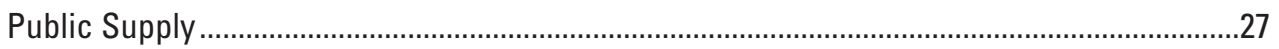

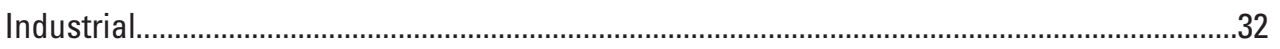

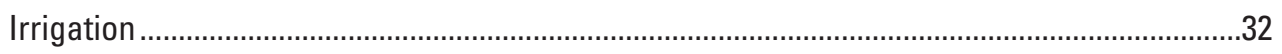

Public-Supply Water Projections for All Reservoir Catchment Areas to 2040 ..................................43

Martins Fork Reservoir Catchment Area .........................................................................50

Laurel Reservoir Catchment Area .......................................................................................51

Wolf Creek Reservoir Catchment Area ............................................................................52

Dale Hollow Reservoir Catchment Area ............................................................................53

Cordell Hull Reservoir Catchment Area ............................................................................54

Center Hill Reservoir Catchment Area ................................................................................5

Old Hickory Reservoir Catchment Area ..........................................................................56

J. Percy Priest Reservoir Catchment Area .......................................................................57

Cheatham Reservoir Catchment Area ..................................................................................5

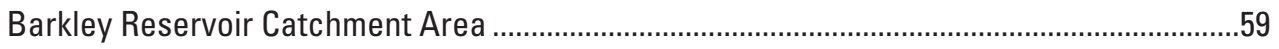

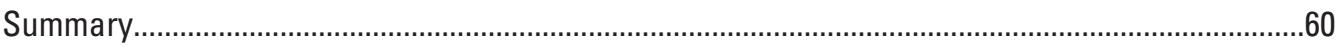

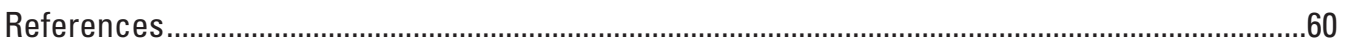

Glossary

\section{Figures}

1. Map showing Cumberland River watershed, major tributaries, and dams, Tennessee and Kentucky

2. Map showing reservoir catchment areas in the Cumberland River watershed...............6

3. Map showing hydrologic units in the Cumberland River watershed.................................

4. Map showing states, counties, and reservoir catchment areas in the Cumberland

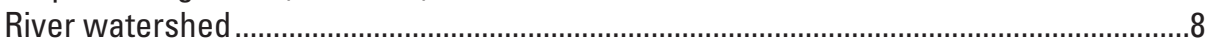

5. Schematic diagram showing source, use, and disposition of water in the Cumberland River watershed in 2010.

6. Maps showing total water withdrawals by source, surface water, groundwater, and total water, and by reservoir catchment area in the Cumberland River watershed in 2010

7. Schematic diagram showing water withdrawals and consumptive use by category, in million gallons per day, in the Cumberland River watershed in 2010 
8. Map showing the locations of thermoelectric power plants and surface-water withdrawals for thermoelectric use, by reservoir catchment area, in the Cumberland River watershed in 2010

9. Maps showing public-supply water withdrawals by source, surface water, groundwater, and total water, and by reservoir catchment area in the Cumberland River watershed in 2010 ..

10. Maps showing industrial water withdrawals by source, surface water, groundwater, and total water, and by reservoir catchment area in the Cumberland River watershed in 2010

11. Maps showing irrigation water withdrawals by source, surface water, groundwater, and total water, and by reservoir catchment area in the Cumberland River watershed in 2010

12. Maps showing public-supply water use for 2010 and projections of publicsupply water withdrawals for 2020, 2030, and 2040 by reservoir catchment area in the Cumberland River watershed.

13. Graph showing public-supply water use by reservoir catchment area in the Cumberland River watershed, 2010 to 2040.

14. Graph showing public-supply water use in 2010 and projected public-supply water use for 2020-2040, Martins Fork reservoir catchment area, Cumberland River watershed.

15. Graph showing public-supply water use in 2010 and projected public-supply water use for 2020-2040, Laurel reservoir catchment area, Cumberland River watershed

16. Graph showing public-supply water use in 2010 and projected public-supply water use for 2020-2040, Wolf Creek reservoir catchment area, Cumberland River watershed

17. Graph showing public-supply water use in 2010 and projected public-supply water use for 2020-2040, Dale Hollow reservoir catchment area, Cumberland River watershed

18. Graph showing public-supply water use in 2010 and projected public-supply water use for 2020-2040, Cordell Hull reservoir catchment area, Cumberland River watershed

19. Graph showing public-supply water use in 2010 and projected public-supply water use for 2020-2040, Center Hill reservoir catchment area, Cumberland River watershed

20. Graph showing public-supply water use in 2010 and projected public-supply water use for 2020-2040, Old Hickory reservoir catchment area, Cumberland River watershed

21. Graph showing public-supply water use in 2010 and projected public-supply water use for 2020-2040, J. Percy Priest reservoir catchment area, Cumberland River watershed..

22. Graph showing public-supply water use in 2010 and projected public-supply water use for 2020-2040, Cheatham reservoir catchment area, Cumberland River watershed

23. Graph showing public-supply water use in 2010 and projected public-supply water use for 2020-2040, Barkley reservoir catchment area, Cumberland River watershed 


\section{Tables}

1. Total offstream water use by reservoir catchment area, Cumberland River watershed, 2010 .

2. Total offstream water use by hydrologic unit, Cumberland River watershed, 2010 ........14

3. Total water use by category, reservoir catchment area, hydrologic unit, and county, Cumberland River watershed, 2010

4. Thermoelectric power water use by reservoir catchment area, hydrologic unit, and county, Cumberland River watershed, 2010

5. Public-supply water withdrawals by reservoir catchment area, hydrologic unit, and county, Cumberland River watershed, 2010

6. Industrial water withdrawals by reservoir catchment area, hydrologic unit, and county, Cumberland River watershed, 2010

7. Irrigation water withdrawals by reservoir catchment area, hydrologic unit, and county, Cumberland River watershed, 2010

8. Public-supply water use, 2010, and projections of public-supply water use, 2020-2040, by reservoir catchment area, Cumberland River watershed

9. Public-supply water use, 2010 , and projections of public-supply water use, 2020-2040, by reservoir catchment area, hydrologic unit, and county, Cumberland River watershed.

10. Population served, public-supply water use during 2010, and public-supply water-use projections in the Martins Fork reservoir catchment area, 2020, 2030, and 2040

11. Population served, public-supply water use during 2010, and public-supply water-use projections in the Laurel reservoir catchment area, 2020, 2030, and 2040 .

12. Population served, public-supply water use during 2010, and public-supply water-use projections in the Wolf Creek reservoir catchment area, 2020, 2030, and 2040

13. Population served, public-supply water use during 2010, and public-supply water-use projections in the Dale Hollow reservoir catchment area, 2020, 2030, and 2040

14. Population served, public-supply water use during 2010, and public-supply water-use projections in the Cordell Hull reservoir catchment area, 2020, 2030 , and 2040

15. Population served, public-supply water use during 2010, and public-supply water-use projections in the Center Hill reservoir catchment area, 2020, 2030, and 2040

16. Population served, public-supply water use during 2010, and public-supply water-use projections in the Old Hickory reservoir catchment area, 2020, 2030, and 2040

17. Population served, public-supply water use during 2010, and public-supply water-use projections in the J. Percy Priest reservoir catchment area, 2020, 2030 , and 2040

18. Population served, public-supply water use during 2010, and public-supply water-use projections in the Cheatham reservoir catchment area, 2020, 2030, and 2040 ..

19. Population served, public-supply water use during 2010, and public-supply water-use projections in the Barkley reservoir catchment area, 2020, 2030, and 2040 . 


\section{Conversion Factors}

U.S. customary units to International System of Units

\begin{tabular}{lll}
\hline \multicolumn{1}{c}{ Multiply } & By & \multicolumn{1}{c}{ To obtain } \\
\hline inch (in.) & Length & \\
mile (mi) & 2.54 & centimeter $(\mathrm{cm})$ \\
\hline & 1.609 & kilometer $(\mathrm{km})$ \\
\hline acre & Area & \\
acre & 43,560 & square foot $\left(\mathrm{ft}^{2}\right)$ \\
acre & 4.047 & square meter $\left(\mathrm{m}^{2}\right)$ \\
square mile (mi $\left.{ }^{2}\right)$ & 0.001562 & square mile $\left(\mathrm{mi}^{2}\right)$ \\
\hline & 2.590 & square kilometer $\left(\mathrm{km}^{2}\right)$ \\
\hline gallons per day $(\mathrm{gal} / \mathrm{d})$ & $\mathrm{Flow}$ & \\
million gallons per day $(\mathrm{Mgal} / \mathrm{d})$ & 3.785 & liters per day $(\mathrm{L} / \mathrm{d})$ \\
million gallons per day $(\mathrm{Mgal} / \mathrm{d})$ & 1.121 & thousand acre-feet per year $(\mathrm{acre}-\mathrm{ft} / \mathrm{yr})$ \\
million gallons per day $(\mathrm{Mgal} / \mathrm{d})$ & 0.001547 & thousand cubic feet per second $\left(\mathrm{ft}^{3} / \mathrm{s}\right)$ \\
million gallons per day $(\mathrm{Mgal} / \mathrm{d})$ & 0.6944 & thousand gallons per minute $(\mathrm{gal} / \mathrm{min})$ \\
million gallons per day $(\mathrm{Mgal} / \mathrm{d})$ & 0.003785 & million cubic meters per day $\left(\mathrm{m}^{3} / \mathrm{d}\right)$ \\
\hline & 1.3815 & million cubic meters per year $\left(\mathrm{m}^{3} / \mathrm{yr}\right)$ \\
\hline kilowatt-hour $(\mathrm{kWh})$ & Energy & \\
\hline
\end{tabular}

Temperature in degrees Fahrenheit $\left({ }^{\circ} \mathrm{F}\right)$ may be converted to degrees Celsius $\left({ }^{\circ} \mathrm{C}\right)$ as follows:

$$
{ }^{\circ} \mathrm{C}=\left({ }^{\circ} \mathrm{F}-32\right) / 1.8
$$

Selected water equivalents in inch-pound units are listed below:

$\begin{array}{rll}1 \text { gallon } & = & 8.34 \text { pounds } \\ 1 \text { million gallons } & = & 3.07 \text { acre-feet } \\ 1 \text { cubic foot } & = & 62.4 \text { pounds } \\ & =\quad 7.48 \text { gallons } \\ 1 \text { acre-foot (acre-ft) } & =\quad 325,851 \text { gallons } \\ & =43,560 \text { cubic feet } \\ 1 \text { inch of rain } & =\quad 17.4 \text { million gallons per square mile } \\ & =\quad 27,200 \text { gallons per acre } \\ & =\quad 100 \text { tons per acre }\end{array}$




\section{Datum}

Horizontal coordinate information is referenced to the North American Datum of 1983 (NAD 83).

\section{Abbreviations}

CBER Center for Business and Economic Research, The University of Tennessee, Knoxville

EPA U.S. Environmental Protection Agency

HUC Hydrologic unit code

RCA Reservoir catchment area

USACE U.S. Army Corps of Engineers

USGS U.S. Geological Survey

WSS Water system survey 


\title{
Estimated Use of Water in the Cumberland River Watershed in 2010 and Projections of Public-Supply Water Use to 2040
}

\author{
By John A. Robinson
}

\section{Abstract}

The U.S. Army Corps of Engineers (USACE), Nashville District, is conducting ongoing water-supply analyses of USACE reservoirs in the Cumberland River watershed to identify areas where potential water-resources issues may arise in the future. To assist the USACE in their efforts, the U.S. Geological Survey, in cooperation with the USACE, collected and analyzed water-use data to estimate public-supply, selfsupplied industrial, irrigation, and thermoelectric water use for 2010 and to project water demand to 2040 for the Cumberland River watershed area.

Estimates of water use for public supply were projected in 10-year increments through 2040 and were based on 2010 public water-supply data and population projections for 2020 to 2040. Additionally, estimates of consumptive use, wastewater releases, and thermoelectric power and industrial return flows were calculated. All estimates are presented for the entire watershed and for the 10 reservoir catchment areas (RCAs) within the watershed.

Estimated water withdrawals in the Cumberland River watershed during 2010 averaged 3,456.23 million gallons per day (Mgal/d) of freshwater for offstream use. Return flow was estimated to be $3,370.08 \mathrm{Mgal} / \mathrm{d}$, or 98 percent of the water withdrawn during 2010. Total consumptive use accounts for the remaining 2 percent, or $86.2 \mathrm{Mgal} / \mathrm{d}$. Estimates of water withdrawals by source indicate that withdrawals from surface water during 2010 accounted for more than 99 percent of the total withdrawals, or $3,437.90 \mathrm{Mgal} / \mathrm{d}$. Total groundwater withdrawals during 2010 were $18.33 \mathrm{Mgal} / \mathrm{d}$, or less than 1 percent of the total withdrawals.

During 2010, withdrawals by category were estimated as follows: thermoelectric power, 3,051.12 Mgal/d; public supply, $360.00 \mathrm{Mgal} / \mathrm{d}$; industrial, $31.5 \mathrm{Mgal} / \mathrm{d}$; and irrigation, 13.6 Mgal/d. Return flows were estimated as thermoelectric power, 3,051.06 Mgal/d, and industrial and public supply, $319.02 \mathrm{Mgal} / \mathrm{d}$. Consumptive use was estimated as thermoelectric power, $0.06 \mathrm{Mgal} / \mathrm{d}$; industrial and public supply, $72.5 \mathrm{Mgal} / \mathrm{d}$; and irrigation, $13.6 \mathrm{Mgal} / \mathrm{d}$.

By 2040, the public supply of raw and (or) finished water to meet demand for the $10 \mathrm{RCAs}$ is projected to increase 48 percent to $532.51 \mathrm{Mgal} / \mathrm{d}$. This projected increase includes an increase from 51.5 to $72.5 \mathrm{Mgal} / \mathrm{d}$, or 41 percent, in the Barkley RCA. The combined total water demand for the Cheatham, J. Percy Priest, and Old Hickory RCAs is projected to increase from 224.08 to $359.58 \mathrm{Mgal} / \mathrm{d}$, or 61 percent. The combined total water demand for the Center Hill, Cordell Hull, and Dale Hollow RCAs is projected to increase from 31.7 to $43.0 \mathrm{Mgal} / \mathrm{d}$, or 36 percent. The combined total water demand for the Martins Fork, Laurel, and Wolf Creek RCAs is projected to increase from 52.8 to $57.4 \mathrm{Mgal} / \mathrm{d}$, or 9 percent. The only RCA in the watershed with a projected decrease in water demand is Martins Fork.

\section{Introduction}

Population growth, moderate to extreme droughts and floods, demands for competing water resources, and aging infrastructure have established the need to evaluate current water use and sustainability of the resources in the Cumberland River watershed of Tennessee and Kentucky. Flow in the Cumberland River system is regulated by a network of 10 major dams and reservoirs that provide water for multiple types of uses. The dams supply hydroelectric power to the Tennessee Valley Authority and other regional power systems. Reservoirs in the watershed provide water storage for public water supplies, commercial navigation, and flood damage reduction; help maintain water quality; and are important for fisheries and wildlife management. The reservoirs also offer recreational, aesthetic, and other waterresources benefits, and much of the economic progress in the watershed relies on these water resources. Maintaining the amount and quality of water in these reservoirs is vital for municipal, industrial, and agricultural growth in the Cumberland River watershed.

Wisely managing the water resources in the watershed and preserving and enhancing the diverse and rich aquatic ecosystem are dependent on accurate and complete information pertaining to the availability and use of the water resources. Reliable information concerning where water is used, how water is used, the quantity of water used, and changes in water use over time is key in making informed water-resources management decisions. Comprehensive 
water-use information, by reservoir catchment area (RCA) or subwatershed (hydrologic unit code [HUC]), is not currently available but is needed by decision makers in the watershed to help them evaluate the effects of current and projected water-use demands on the available water resources. In the coming decades, growth and development in the watershed and surrounding areas will continue to affect water resources, and the ability to understand and manage the effects of future demand on ecosystems, water availability, and water quality will be important.

The U.S. Geological Survey (USGS), in cooperation with the U.S. Army Corps of Engineers (USACE), conducted an investigation to collect and analyze water-use data for 2010 and to project water demand to 2040 for the Cumberland River watershed area. These data will be used by the USACE as part of ongoing water-supply analyses for USACE reservoirs in the watershed and will help to identify areas where potential water-resources issues may arise. The analyses presented in this report directly support strategic science goals of the USGS, notably improving the understanding of the quantity of water needed as human and environmental needs change over time (USGS, 2007). These estimates are being presented by the USGS to assist the USACE in determining the effect of existing and future water use on the ability of USACE reservoirs to provide authorized benefits (flood damage reduction, hydropower production, commercial navigation, recreation opportunities, water-quality maintenance, and fish and wildlife preservation) and to determine whether changes in the USACE reservoir operating plans to accommodate water use would produce greater overall public value.

\section{Purpose and Scope}

The purpose of this report is to provide water-use estimates for the Cumberland River watershed for 2010 and water-use trends and projections in 10-year increments through 2040. Elements of this report include the estimation of public water supply, self-supplied industrial water supply, irrigation water use, and thermoelectric water use in 2010 by RCA, HUC, and county. Estimated water use for public water supply is projected in 10-year increments through 2040 based on population projections. Additional elements of the report include estimates of consumptive use, wastewater releases, and thermoelectric power and industrial return flows. All data used to support the analysis and conclusions presented in this report are publicly available from Robinson (2017).

\section{Hydrologic Setting}

The Cumberland River watershed extends from Harlan, Kentucky, through three major cities in Middle Tennessee, to near Smithland, Kentucky, at the confluence of the highly regulated Cumberland River and Ohio River (fig. 1). The headwaters of the Cumberland River are in the mountains of southeastern Kentucky. The Cumberland River is formed by the confluence of Poor Fork, Clover Fork, and Martins Fork. The river flows to the west and is fed by Rockcastle River, Buck Creek, and Big South Fork Cumberland River. As the Cumberland River meanders to the southwest, two other tributaries, the Wolf and Obey Rivers, contribute to the flow. Continuing west, the Caney Fork, Stones, and Harpeth Rivers join the Cumberland River. As the Cumberland River flows to the northwest, the Red and Little Rivers contribute to the flow before it eventually joins the Ohio River at Smithland, Kentucky. The entire watershed has a 17,900-square-mile $\left(\mathrm{mi}^{2}\right)$ drainage area and is part of the larger Ohio waterresources region. The Cumberland water-resources region corresponds to the Cumberland River watershed (fig. 1). Most of the watershed is in Tennessee (69 percent or 12,350 $\mathrm{mi}^{2}$ ) with the remainder in Kentucky (31 percent or 5,550 $\mathrm{mi}^{2}$ ).

Ten dams-Martins Fork, Laurel, Wolf Creek, Dale Hollow, Cordell Hull, Center Hill, Old Hickory, J. Percy Priest, Cheatham, and Barkley - constitute the USACE Cumberland River Basin water-control system (fig. 1). The reservoirs are operated year-round for the purposes of navigation, flood damage reduction, power generation, water supply, water quality, fish and wildlife, and recreation.

About 2.4 million people resided in the watershed in 2010 (U.S. Census Bureau, 2010a), and the most populated cities were Nashville $(627,000)$, Clarksville $(133,000)$, and Murfreesboro (109,000), Tennessee (U.S. Census Bureau, 2010b).

\section{Climate}

Climate in the watershed generally is temperate, warm, and humid. Annual average temperature is 56.9 degrees Fahrenheit, and the coldest and warmest months are January and July, respectively. The average annual precipitation was 51.8 inches during 1981 to 2010 , with an average of 5.38 inches occurring in May, the wettest month, and an average of 3.47 inches occurring in October, the driest month (National Oceanic and Atmospheric Administration, 2013). Precipitation in the form of short thunderstorms occurs from late spring through early fall; however, storms from December through May can last for several days and produce large amounts of precipitation.

Since 2007, parts of Kentucky and Tennessee have experienced record floods and droughts. Moderate to extreme droughts occurred in the spring, summer, and fall of 2007 and 2008 and in the summer of 2012 (U.S. Drought Monitor, 2007, 2008, 2012). Moderate to extreme flooding occurred in the spring of 2010 (National Oceanic and Atmospheric Administration, 2010) and 2011 (National Oceanic and Atmospheric Administration, 2011).

In general, the seasonal climate affects water-use patterns (Lins and others, 2010), and the operation of the reservoirs is linked to rainfall and runoff patterns. Typically, water use increases during hot and dry summers and decreases during cool and wet winters. Seasonal variability is also affected by behavioral patterns such as tourism and the use of water 


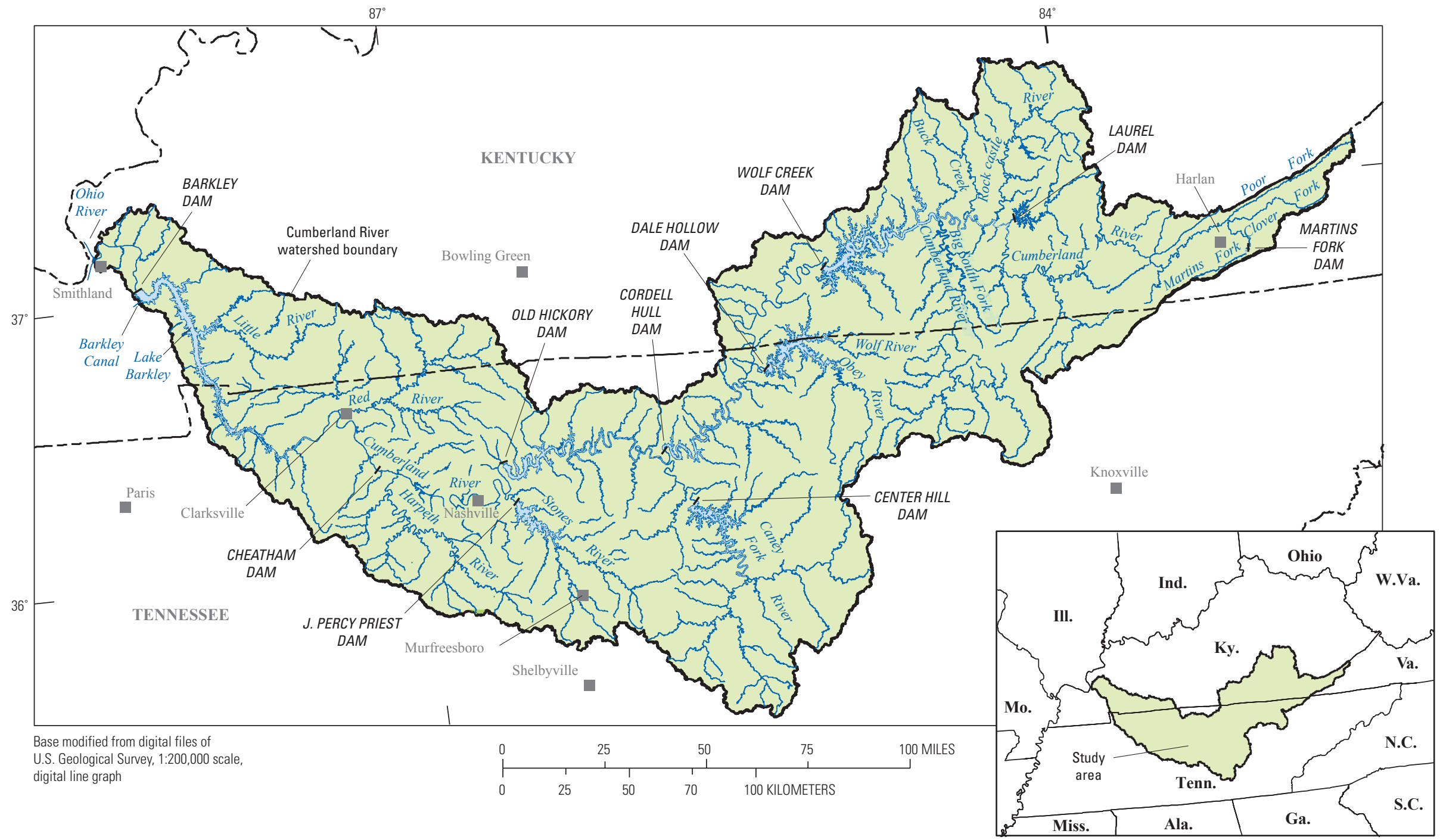

Location of the study area in Tennessee and Kentucky

Figure 1. Cumberland River watershed, major tributaries, and dams, Tennessee and Kentucky. 
for lawns, gardens, and swimming pools. If the winter temperatures remain below freezing for extended periods of time, water use can increase because of the need, in some cases, to leave water running or because of leaks as frozen pipes thaw. Periodic droughts have increased the need for required conservation measures and sometimes have resulted in water-use restrictions.

\section{Data Compilation and Analysis}

The type and availability of water-use data vary by State and are determined by State law, the presence of a waterpermitting or water-use program, and funding. The 2010 water-use data presented in this report were compiled from various sources, depending on the category of use and the information available for each State and were aggregated by USGS personnel from Kentucky and Tennessee and the USGS National Water-Use Science Project. USGS personnel determined the best sources of information available, then compiled or estimated the data and prepared documentation of the sources and methods used to determine the water-use totals. Consequently, the data in this report may have been derived from reported, estimated, or calculated means by using different sources and methods and, therefore, will have varying levels of accuracy (Maupin and others, 2014). The water-use data were reviewed and revised, where needed, based on quality-assurance and quality-control checks.

The USGS conducted a supplementary survey to obtain water-withdrawal and return-flow data from thermoelectric power and industrial facilities located in the watershed. The U.S. Department of Energy, Energy Information Administration (DOI, EIA) electricity database was a secondary source of information on water withdrawal, return flow, and power generation for the thermoelectric plants (DOE, EIA, 2010a, b). Estimates of industrial withdrawals by facilities with total usage values equal to and greater than 10,000 gallons per day (gal/d) were obtained from State agencies that issued water-use permits or from the water-use survey conducted in conjunction with this investigation. For this report, return flow is limited to cooling-water discharge and excludes stormwater runoff. The municipal wastewater data, industrial sanitary, process, and cooling-water data, and the return-flow data are from the U.S. Environmental Protection Agency (EPA), National Pollutant Discharge Elimination System, Permit Compliance System.

A description of the approach and methods for the collection and analyses of the monthly operation reports containing public-supply data and population served for public-supply water systems located in Tennessee is documented in Robinson and Brooks (2010). Public-supply data and population served by county for public-supply water systems located in Tennessee were obtained from the Tennessee Department of Environment and Conservation, Division of Water Resources (Robinson, 2017). Public-supply data and population served by county for public-supply water systems located in Kentucky were collected as part of the 2010 National Water Use Program (Maupin and others, 2014).

Water-use data for irrigation were compiled from the U.S. Department of Agriculture, National Agricultural Statistic Service Census of Agriculture (USDA, 2013), the Farm and Ranch Irrigation Survey (USDA, 2010), and USGS National Water-Use Science Project. The Census of Agriculture is conducted every 5 years to provide comprehensive agriculture data by State and county. The irrigation data compiled during the 2007 Census of Agriculture were used to calculate the number of acres irrigated, by county, for Tennessee and Kentucky (USDA, 2009a, b). Irrigation data were not available in the Census of Agriculture for seven counties in Tennessee and six counties in Kentucky to avoid the release of confidential information. In the cases where 2007 irrigation data were not available, irrigation data by county from the 2005 USGS national compilation (Kenny and others, 2009) were used. The acreage irrigated in each county was converted to water use by an average application rate per acre (USDA, 2010) for each State, and then irrigation use was converted to million gallons per day. Irrigation use, however, is mostly during the growing season, and actual daily production can be much higher than the annual average water use. The distribution of irrigation by source water, either groundwater or surface water, was not available through the U.S. Department of Agriculture and was based on the sources of irrigation reported in the USGS compilation of water use in the United States for 2005 (Kenny and others, 2009). Golf course water-use values greater than 10,000 gal/d were included in the estimates of irrigation water use for counties in Kentucky. Golf course water-use values greater than $10,000 \mathrm{gal} / \mathrm{d}$ were included in the estimates of industrial water use for counties in Tennessee.

Population data for 2010 were compiled from the U.S. Census Population and Housing Counts for Kentucky (U.S. Census Bureau, 2012a) and Tennessee (U.S. Census Bureau, 2012b). The population for the Cumberland River watershed was estimated by using data from the U.S. Census Bureau for census tracts along with a geographic information system. For census tracts intersected by the Cumberland River watershed boundary, the population of the census tract within the watershed was estimated based on the percentages of area of the census tract contained within the watershed boundary. The sum of the population of the portion of all intersected census tracts and all non-intersected census tracts within the Cumberland River watershed equals the total population in the Cumberland River watershed (D.E. Ladd, U.S. Geological Survey, oral commun., 2013).

Water-use data were aggregated into the 10 RCAs, $14 \mathrm{HUC}$ areas, and by county in the watershed. Ten RCAs (Martins Fork, Laurel, Wolf Creek, Dale Hollow, Cordell Hull, Center Hill, Old Hickory, J. Percy Priest, Cheatham, and Barkley) constitute the Cumberland River watershed (fig. 2). An RCA is a natural drainage area truncated by a dam, and the reservoir is a functional unit operated to meet specific 
objectives ranging from power generation to recreation. The guidelines under which a reservoir is operated are part of an integrated management plan of the entire reservoir system, thus linking water availability throughout the watershed.

The RCAs account for the complete site-specific water-use transactions between adjoining RCAs and are used to determine consumptive use.

Fourteen watershed areas designated by HUC constitute the Cumberland River watershed (fig. 3). The water-use data were aggregated by HUCs because these units are often used as a geographical framework for detailed water-resources planning and for evaluating interbasin transfer of water or wastewater. The areal extent of the sum of all RCAs is less than the areal extent of the Cumberland River watershed. Consequently, estimates of water use may differ when comparing between HUC and RCA. The State and county data are important data-analysis units used in formulating policy and making water-management decisions; therefore, these data are also included in the report. Several counties in the Cumberland River watershed are only partially located within the watershed (fig. 4). For each of these counties, only the water-use transactions occurring within the watershed were compiled for this study.

Net water demand was calculated by subtracting return flow from withdrawals and was determined for each RCA. The net water demand is accumulated at the downstream boundary of the RCA to calculate a consumptive use. Cumulative consumptive use was calculated at key outlets of the RCAs in the river system, resulting in a sum of consumptive use in the watershed for each outlet. Cumulative consumptive use for the Cumberland River watershed was calculated at Barkley Dam. Although calculations for interbasin transfers are beyond the scope of this study, it is important to note that the diversions of water to Kentucky Lake (outside of study area) through Barkley Canal from Lake Barkley are interbasin transfers to the river system (F. Powell, Tennessee Valley Authority, written commun., September 2010).

Water-use transactions are related to sources of supply. Surface-water and groundwater withdrawals and consumptive-use estimates are reported for thermoelectric power, industry, public supply, and irrigation. Wastewater releases are reported as return flows in the public-supply category. Wastewater releases refer to water released from private and public wastewater treatment facilities designated by the EPA as publicly owned treatment works and do not include the quantity of water reclaimed for beneficial uses, such as irrigation of golf courses and parks. Return flow also is reported for the thermoelectric power and industrial categories.

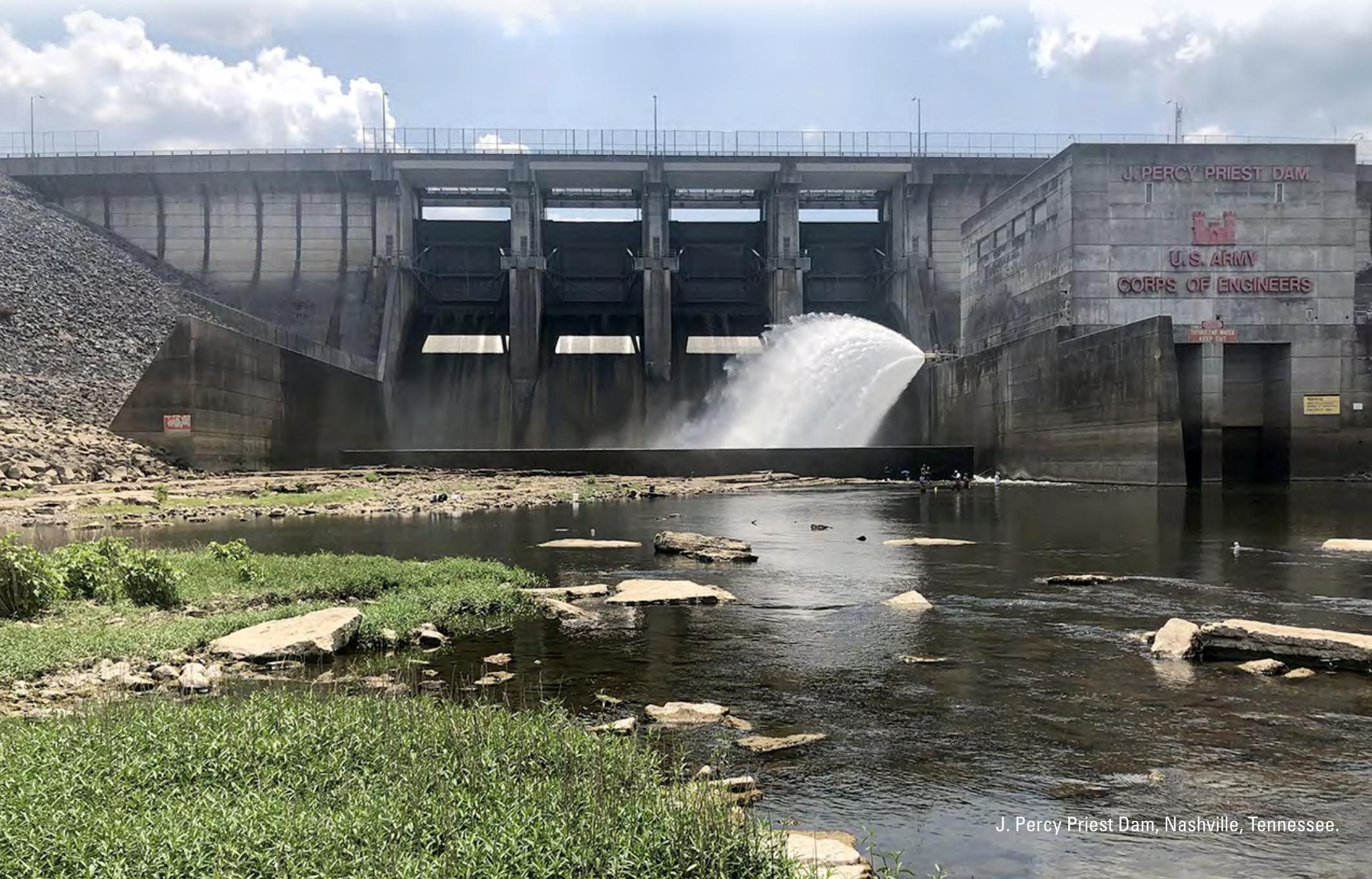




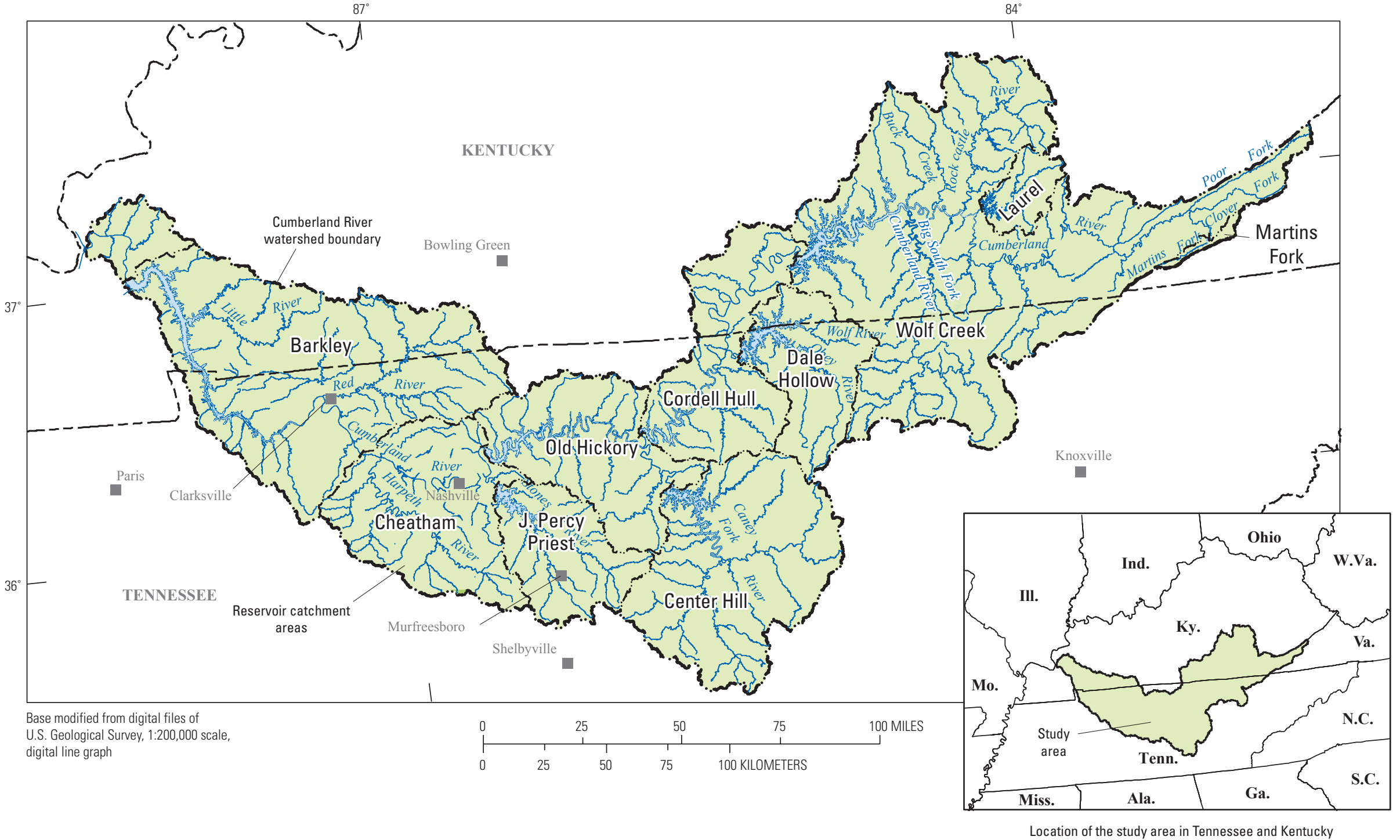

Figure 2. Reservoir catchment areas in the Cumberland River watershed. 


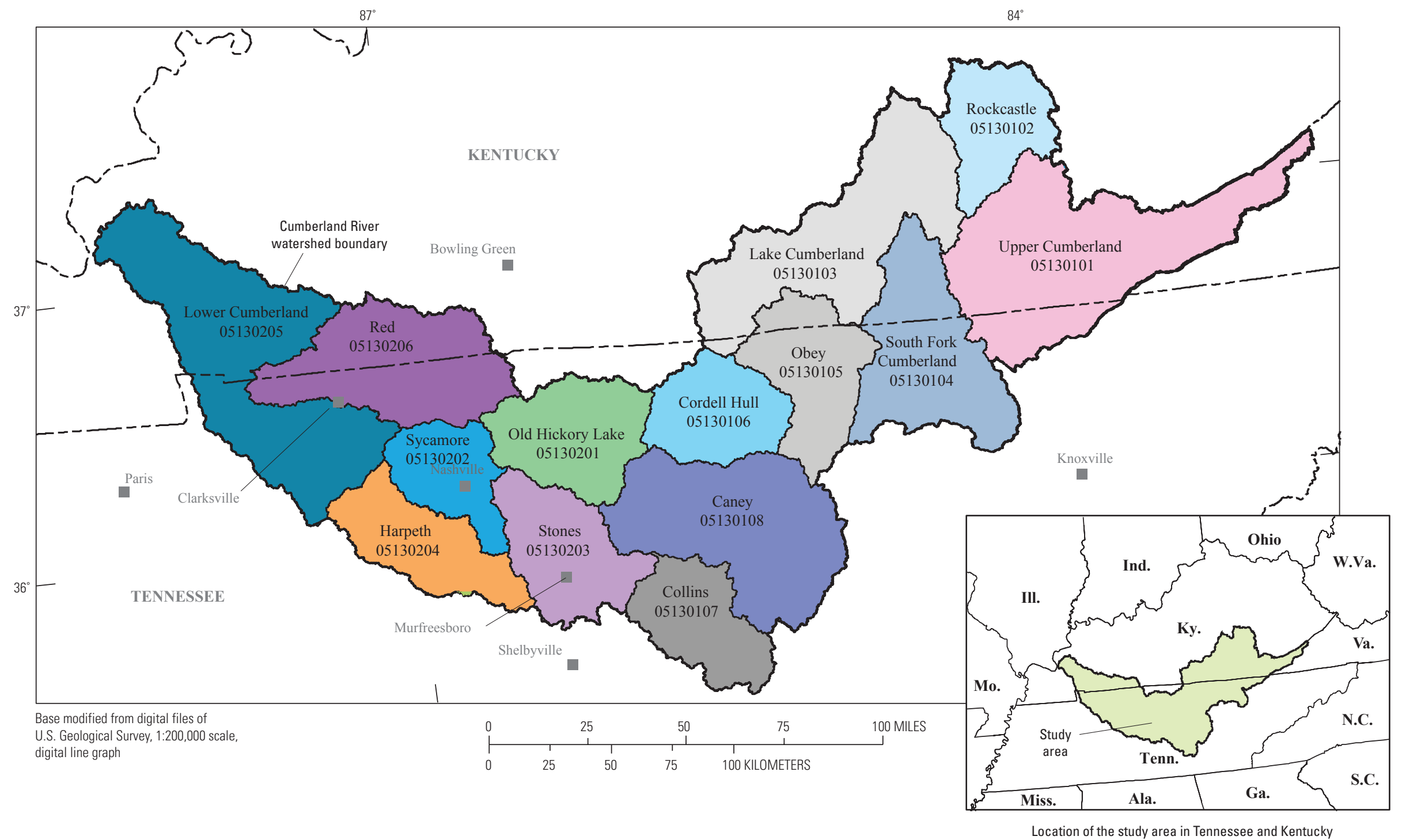

Figure 3. Hydrologic units in the Cumberland River watershed. 


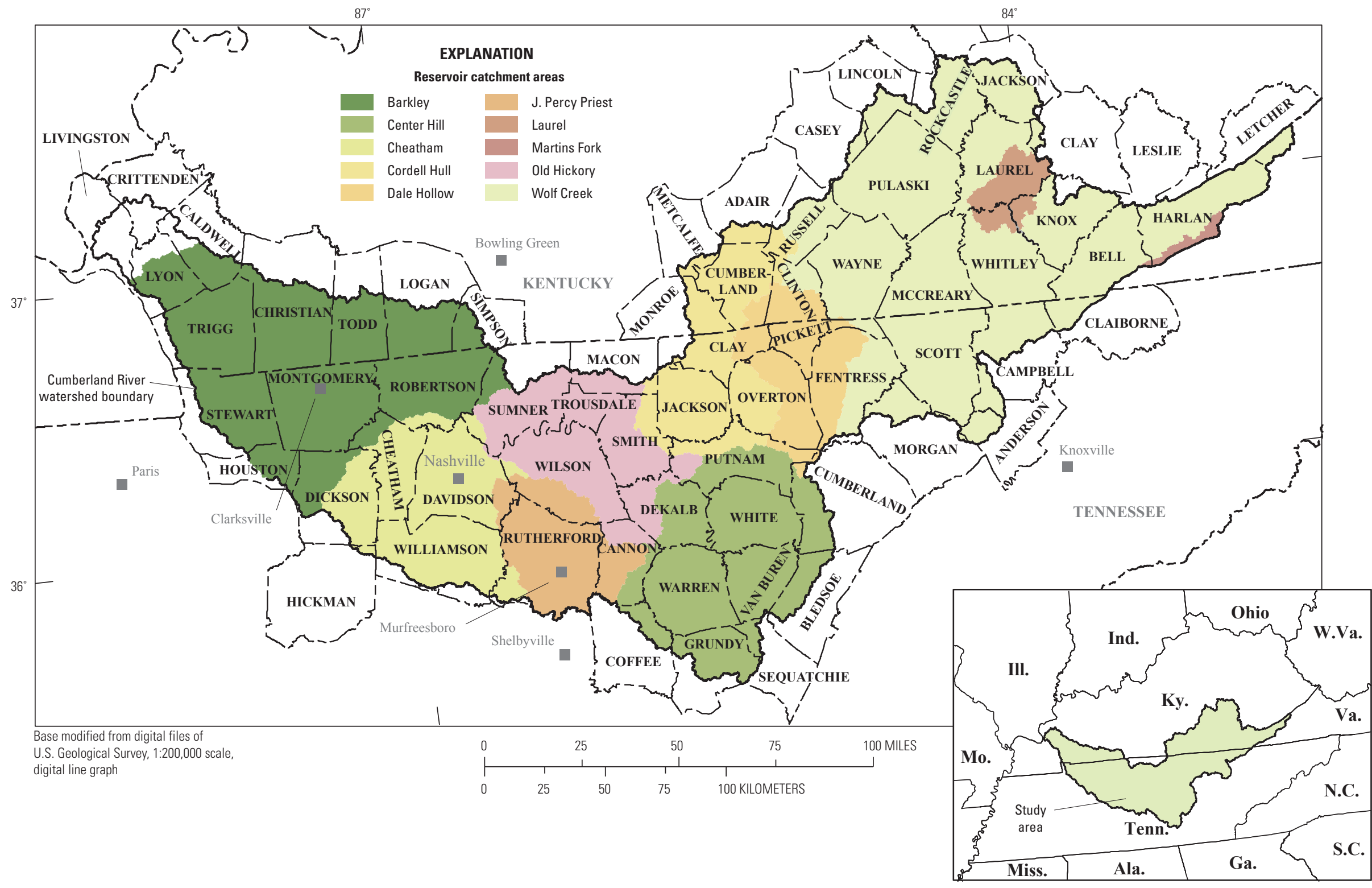

Location of the study area in Tennessee and Kentucky

Figure 4. States, counties, and reservoir catchment areas in the Cumberland River watershed. 


\section{Projections of Water Use}

The methods used to project water use in Tennessee and Kentucky differ because of differences in available data. Water-use projections for the Tennessee water-supply systems are dependent on population served, population density, and the ratio of commercial water use to residential water use, whereas water-use projections for Kentucky are dependent on raw water withdrawals for each public-supply water system and decadal county-level growth projection factors. The reported data collected from the Tennessee Department of Environment and Conservation, Division of Water Resources water system surveys (WSSs) about wateruse data for Tennessee in 2010 and monthly operation reports were used to identify residential, commercial, and industrial deliveries from the public water systems (Robinson, 2017). The population projections used and methods used as the basis for the water-use projections were documented by the Center for Business and Economic Research (CBER) at The University of Tennessee, Knoxville (CBER; 2012) and by the Kentucky State Data Center (KSDC; 2012). The population projections by the CBER and Kentucky State Data Center are primarily based on the cohort-component method (U.S. Census Bureau, 2011), which relies on population measures such as the initial population from the most recent decennial census, historical fertility, mortality, and migration rates, and county population data. Robinson (2017) contains the county population projections and calculated decadal county-level growth projection factors.

The projected increases in residential deliveries were calculated based on per capita water use and projected population increases. Commercial and industrial use were combined, and the combined water-use projections were related to growth in population served and county population density. Statewide in 2005, the ratio of commercial to residential water use tended to increase as county-population density increased up to about 1,000 people per square mile. The ratio of combined commercial and industrial to residential water use (commercial rate adjustment) as a function of population density was used to derive projected commercial and industrial water use. These estimates were combined with residential water-use projections to determine overall water-use projections for Tennessee water systems. Robinson (2017) provides a detailed summary, by RCA, of selected demographics and public-supply water use in 2010 ; public-supply water use in 2010 and projected public-supply water use for 2020-2040; and a sample calculation of wateruse projections for a public-supply water system.

WSSs on 2010 water-use data were not available for 11 public-supply water systems located in Tennessee. In the cases where WSSs on 2010 water-use data were not available, WSSs on 2005 water-use data for Tennessee were used in this study (Robinson, 2017). No WSSs were collected for the study period in Kentucky. Therefore, public-supply water-use projections for Kentucky were computed by multiplying raw water withdrawals for each public-supply water system by decadal county-level growth projection factors.

\section{Estimated Use of Water in the Cumberland River Watershed in 2010}

Total freshwater withdrawals during 2010 were estimated to be 3,456.23 million gallons per day (Mgal/d) for the offstream categories of thermoelectric power, industry, public supply, and irrigation (table 1; fig. 5). Estimates of withdrawals by source indicate that during 2010, total surfacewater withdrawals were more than 99 percent of the total, or $3,437.90 \mathrm{Mgal} / \mathrm{d}$. Total groundwater withdrawals were $18.33 \mathrm{Mgal} / \mathrm{d}$, less than 1 percent of the total. Return flows to streams from thermoelectric power, industrial, and municipal wastewater facilities were estimated to be $3,370.08 \mathrm{Mgal} / \mathrm{d}$, or 98 percent of the water withdrawn during 2010. Consumptive use (total freshwater withdrawals minus total return flow) was $86 \mathrm{Mgal} / \mathrm{d}$, about 2.5 percent of the total water withdrawn. When excluding thermoelectric power totals, water withdrawals were estimated as $405.11 \mathrm{Mgal} / \mathrm{d}$ and return flows as $319.02 \mathrm{Mgal} / \mathrm{d}$.

The Barkley $(2,454.51 \mathrm{Mgal} / \mathrm{d})$ and Old Hickory (621.23 Mgal/d) RCAs accounted for 89 percent of the total water withdrawn from the Cumberland River watershed in 2010 (table 1; fig. 6). These withdrawals were primarily surface-water withdrawals for fossil-fuel thermoelectric plants. A similar comparison of the total withdrawals by HUC (table 2) indicates that 05130205 Lower Cumberland $(2,440.19 \mathrm{Mgal} / \mathrm{d})$ and 05130201 Old Hickory Lake (619.25 Mgal/d) accounted for 89 percent of the total withdrawals. These withdrawals also were primarily for fossilfuel thermoelectric plants.

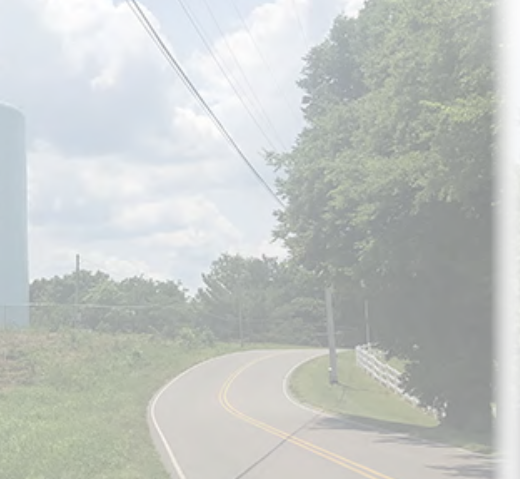

New residential subdivision along bank of Cumberland River, Nashville, Tennessee. 
Table 1. Total offstream water use by reservoir catchment area, Cumberland River watershed, 2010.

[Values may not add to totals because of independent rounding. All values in million gallons per day. Surface water from Martins Fork and Laurel drains into Wolf Creek. Net water demand and consumptive use expressed as integers. RCA, reservoir catchment area. Areal extent of all reservoir catchment areas is less than the areal extent of the Cumberland River watershed]

\begin{tabular}{|c|c|c|c|c|c|c|c|c|c|}
\hline \multirow{3}{*}{ RCA } & \multicolumn{9}{|c|}{ Water withdrawals } \\
\hline & \multicolumn{3}{|c|}{ Public supply } & \multicolumn{3}{|c|}{ Industrial } & \multicolumn{3}{|c|}{ Irrigation } \\
\hline & $\begin{array}{c}\text { Surface } \\
\text { water }\end{array}$ & $\begin{array}{l}\text { Ground- } \\
\text { water }\end{array}$ & Total & $\begin{array}{c}\text { Surface } \\
\text { water }\end{array}$ & $\begin{array}{c}\text { Ground- } \\
\text { water }\end{array}$ & Total & $\begin{array}{c}\text { Surface } \\
\text { water }\end{array}$ & $\begin{array}{c}\text { Ground- } \\
\text { water }\end{array}$ & Total \\
\hline Martins Fork & 2.30 & 0.00 & 2.30 & 0.00 & 0.00 & 0.00 & 0.0003 & 0.00 & 0.0003 \\
\hline Laurel & 8.83 & 0.00 & 8.83 & 0.00 & 0.00 & 0.00 & 0.009 & 0.00 & 0.009 \\
\hline Dale Hollow & 1.89 & 0.008 & 1.90 & 0.00 & 0.00 & 0.00 & 0.007 & 0.092 & 0.099 \\
\hline Cordell Hull & 4.72 & 0.00 & 4.72 & 0.210 & 0.037 & 0.247 & 0.045 & 0.172 & 0.217 \\
\hline \multicolumn{10}{|l|}{ Cumulative consumptive use } \\
\hline \multicolumn{10}{|l|}{ Cumulative consumptive use } \\
\hline J. Percy Priest & 34.9 & 1.71 & 36.6 & 0.469 & 0.016 & 0.485 & 0.003 & 0.973 & 0.976 \\
\hline Cheatham & 143.85 & 0.112 & 143.97 & 8.85 & 0.436 & 9.29 & 0.135 & 1.17 & 1.31 \\
\hline \multicolumn{10}{|l|}{ Cumulative consumptive use } \\
\hline Barkley & 47.5 & 4.16 & 51.6 & 2.82 & 0.100 & 2.92 & 2.27 & 2.19 & 4.46 \\
\hline \multicolumn{10}{|l|}{ Cumulative consumptive use } \\
\hline
\end{tabular}


Table 1. Total offstream water use by reservoir catchment area, Cumberland River watershed, 2010.—Continued

[Values may not add to totals because of independent rounding. All values in million gallons per day. Surface water from Martins Fork and Laurel drains into Wolf Creek. Net water demand and consumptive use expressed as integers. RCA, reservoir catchment area. Areal extent of all reservoir catchment areas is less than the areal extent of the Cumberland River watershed]

\begin{tabular}{|c|c|c|c|c|c|c|c|c|}
\hline \multirow{3}{*}{ RCA } & \multicolumn{4}{|c|}{ Water withdrawals } & \multirow{2}{*}{\multicolumn{3}{|c|}{ Return flow }} & \multirow{3}{*}{$\begin{array}{c}\text { Net water } \\
\text { demand } \\
\text { (consumptive } \\
\text { use) }\end{array}$} \\
\hline & \multicolumn{3}{|c|}{ Thermoelectric } & \multirow[b]{2}{*}{ Total } & & & & \\
\hline & $\begin{array}{l}\text { Surface } \\
\text { water }\end{array}$ & $\begin{array}{l}\text { Ground- } \\
\text { water }\end{array}$ & Total & & Nonpower & Power & Total & \\
\hline Martins Fork & 0.00 & 0.00 & 0.00 & 2.30 & 0.00 & 0.00 & 0.00 & 2 \\
\hline Laurel & 0.00 & 0.00 & 0.00 & 8.84 & 9.68 & 0.00 & 9.68 & -1 \\
\hline Wolf Creek & 96.4 & 0.00 & 96.4 & 138.45 & 18.3 & 96.4 & 114.63 & 24 \\
\hline Cumulative consumptive use & & & & & & & & 25 \\
\hline Dale Hollow & 0.00 & 0.00 & 0.00 & 2.00 & 0.635 & 0.00 & 0.635 & 1 \\
\hline Cordell Hull & 0.00 & 0.00 & 0.00 & 5.18 & 0.516 & 0.00 & 0.516 & 5 \\
\hline Cumulative consumptive use & & & & & & & & 31 \\
\hline Center Hill & 0.00 & 0.00 & 0.00 & 29.73 & 11.1 & 0.00 & 11.1 & 19 \\
\hline Old Hickory & 559.20 & 0.00 & 559.20 & 621.23 & 35.6 & 559.20 & 594.78 & 26 \\
\hline Cumulative consumptive use & & & & & & & & 76 \\
\hline J. Percy Priest & 0.00 & 0.00 & 0.00 & 38.08 & 16.7 & 0.00 & 16.7 & 21 \\
\hline Cheatham & 0.00 & 0.00 & 0.00 & 154.56 & 168.91 & 0.00 & 168.91 & -14 \\
\hline Cumulative consumptive use & & & & & & & & 83 \\
\hline Barkley & $2,395.50$ & 0.00 & $2,395.50$ & $2,454.51$ & 57.5 & $2,395.50$ & $2,453.05$ & 1 \\
\hline Cumulative consumptive use & & & & & & & & 85 \\
\hline RCA total & $3,051.12$ & 0.00 & $3,051.12$ & $3,454.88$ & 319.02 & $3,051.06$ & $3,370.08$ & \\
\hline Kentucky total & 96.4 & 0.00 & 96.4 & 171.68 & 37.3 & 96.4 & 133.66 & \\
\hline Tennessee total & $2,954.70$ & 0.00 & $2,954.70$ & $3,284.55$ & 281.71 & $2,954.70$ & $3,236.41$ & \\
\hline $\begin{array}{l}\text { Cumberland River watershed } \\
\text { total }\end{array}$ & $3,051.12$ & 0.00 & $3,051.12$ & $3,456.23$ & 319.02 & $3,051.06$ & $3,370.08$ & 86 \\
\hline
\end{tabular}




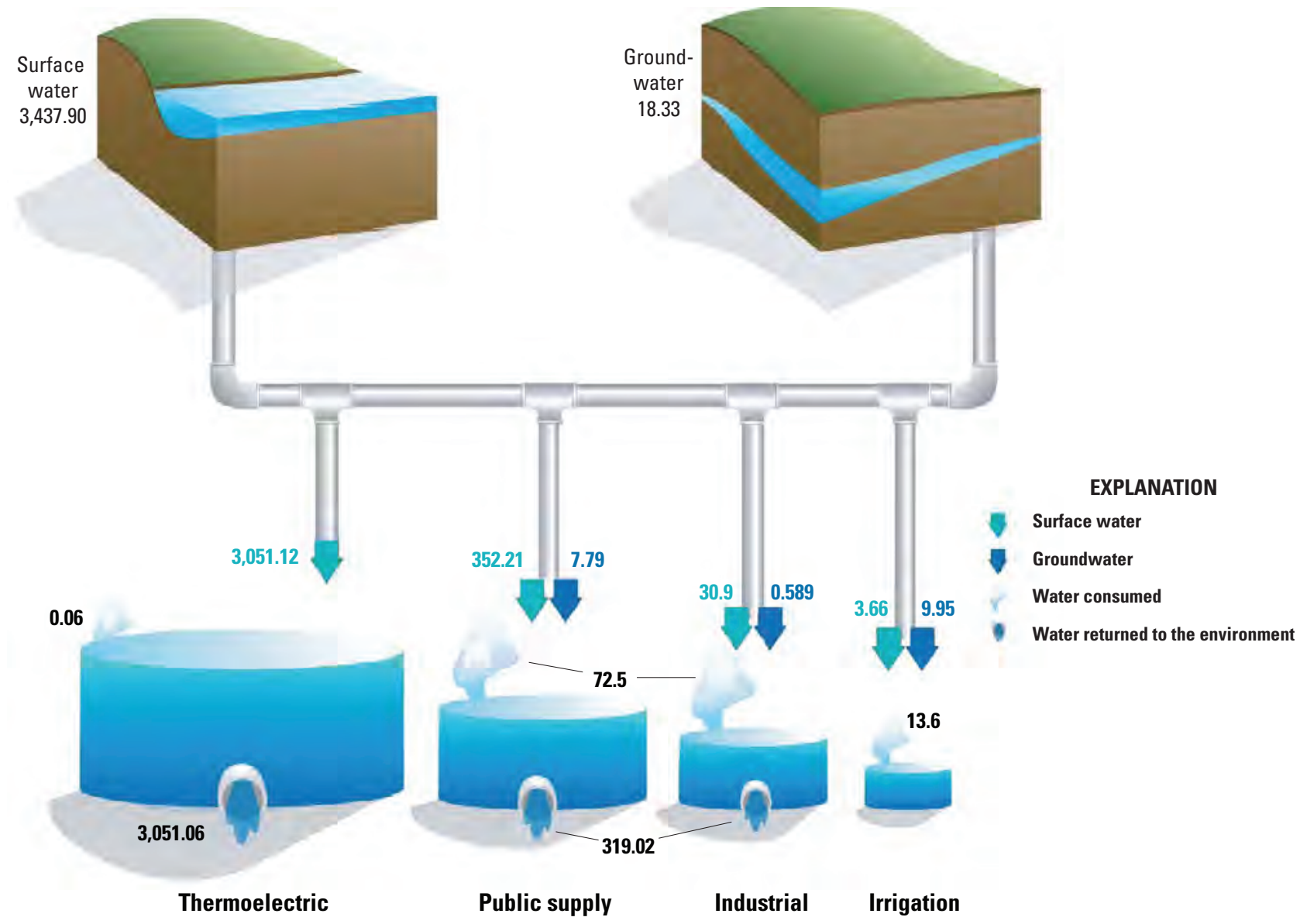

Note: Values are in million gallons per day. Modified from Hutson and others, 2003. Values may not add to totals because of rounding. Schematic not to scale.

Figure 5. Source, use, and disposition of water in the Cumberland River watershed in 2010. 


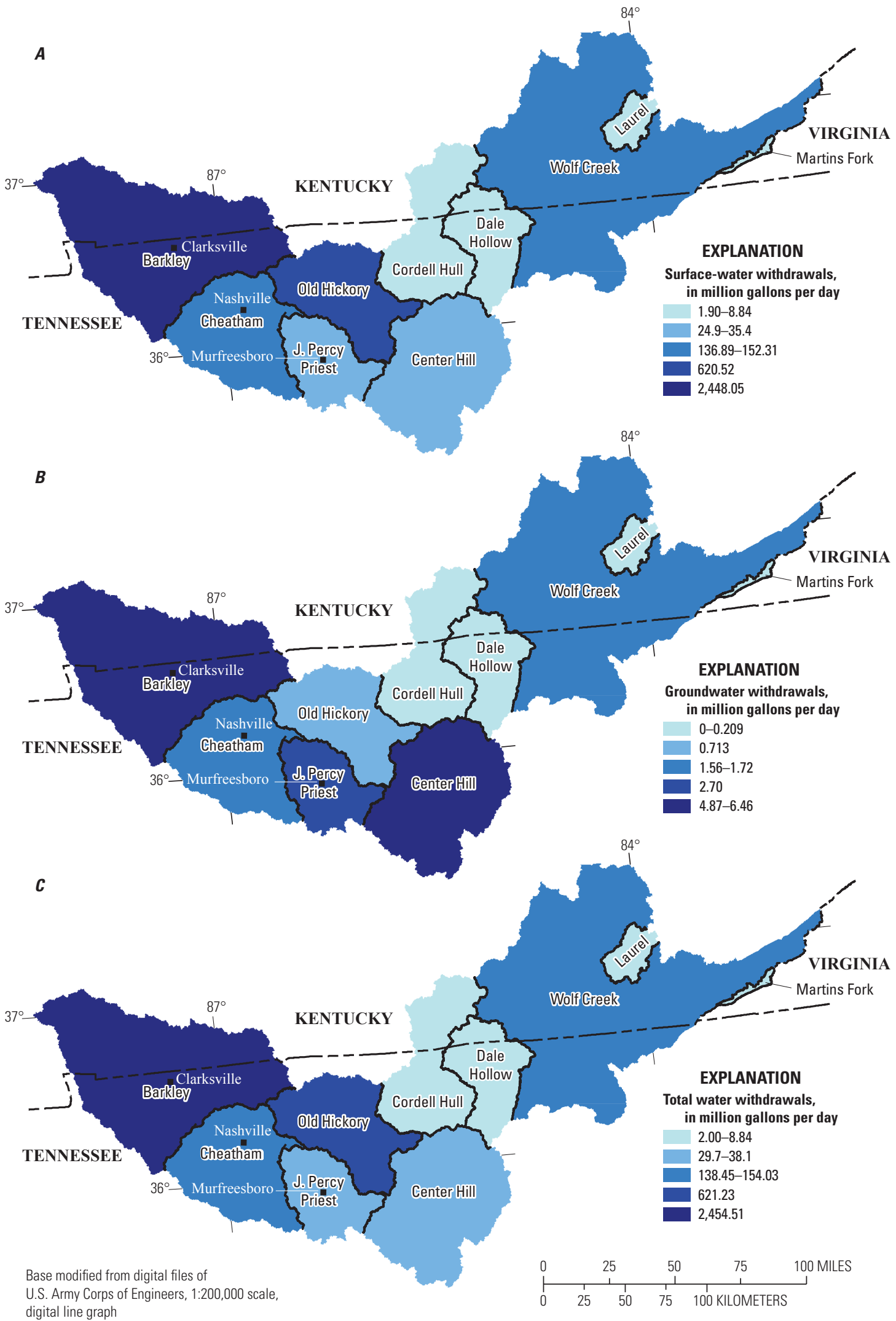

Figure 6. Total water withdrawals by source, $(A)$ surface water, $(B)$ groundwater, and $(C)$ total water, and by reservoir catchment area in the Cumberland River watershed in 2010. 
Table 2. Total offstream water use by hydrologic unit, Cumberland River watershed, 2010.

[Values may not add to totals because of independent rounding. All values in million gallons per day. $<$, less than; net water demand expressed as integers; $<0.001$, actual values less than 0.001 not in table but used to sum totals. Areal extent of all hydrologic units is equal to the areal extent of the Cumberland River watershed]

\begin{tabular}{|c|c|c|c|c|c|c|}
\hline \multirow{2}{*}{\multicolumn{2}{|c|}{$\begin{array}{l}\text { Hydrologic unit } \\
\text { (See fig. 3) }\end{array}$}} & \multicolumn{3}{|c|}{ Water withdrawals } & \multirow{3}{*}{$\begin{array}{c}\begin{array}{c}\text { Total return } \\
\text { flow }\end{array} \\
21.2\end{array}$} & \multirow{3}{*}{$\begin{array}{c}\begin{array}{c}\text { Net water } \\
\text { demand }\end{array} \\
-1\end{array}$} \\
\hline & & \multirow{2}{*}{$\begin{array}{c}\text { Surface water } \\
19.0\end{array}$} & \multirow{2}{*}{$\begin{array}{c}\text { Groundwater } \\
1.53\end{array}$} & \multirow{2}{*}{\begin{tabular}{r|} 
Total \\
20.5
\end{tabular}} & & \\
\hline Upper Cumberland & 05130101 & & & & & \\
\hline Lake Cumberland & 05130103 & 116.19 & 0.003 & 116.20 & 102.58 & 14 \\
\hline South Fork Cumberland & 05130104 & 5.37 & 0.025 & 5.39 & 0.003 & 5 \\
\hline Cordell Hull Reservoir & 05130106 & 3.46 & 0.210 & 3.67 & 0.00 & 4 \\
\hline Collins & 05130107 & 6.66 & 4.21 & 10.9 & 5.31 & 6 \\
\hline Caney & 05130108 & 20.0 & 0.827 & 20.8 & 5.83 & 15 \\
\hline Old Hickory Lake & 05130201 & 618.71 & 0.541 & 619.25 & 594.78 & 24 \\
\hline Sycamore & 05130202 & 146.38 & 0.955 & 147.34 & 163.90 & -17 \\
\hline Red & 05130206 & 10.4 & 5.23 & 15.7 & 26.2 & -11 \\
\hline Cumberland River watershed total & & $3,437.90$ & 18.33 & $3,456.23$ & $3,370.14$ & 86 \\
\hline
\end{tabular}

During 2010, the two largest categories of withdrawals were thermoelectric and public supply (table 3 ). Eighty-nine percent of the total withdrawals $(3,051.12 \mathrm{Mgal} / \mathrm{d})$ was for cooling at thermoelectric plants. The largest thermoelectric power water withdrawal $(2,395.50 \mathrm{Mgal} / \mathrm{d}, 79$ percent of total thermoelectric withdrawals) was from the Barkley RCA. Public-supply withdrawals (table 1; $360 \mathrm{Mgal} / \mathrm{d}$ ) accounted for 10 percent of the total withdrawals. The largest public-supply withdrawal, $143.97 \mathrm{Mgal} / \mathrm{d}$, was from the Cheatham RCA and was 40 percent of the total public-supply withdrawals.

The largest return flow, 2,453.05 Mgal/d, was from the Barkley RCA and accounted for 73 percent of the total return flow. Overall, thermoelectric power had the smallest consumptive use because water withdrawn by thermoelectric power plants was used for cooling, and most of the water was returned to the Cumberland River. Less than 1 percent, or $0.06 \mathrm{Mgal} / \mathrm{d}$, of thermoelectric power water withdrawals were consumptively used, compared to the combined total of 86.1 Mgal/d for industry, public supply, and irrigation (fig. 7).
Consumptive use is reported as an increasing number at the junctures of the RCAs to show the influence of cumulative water withdrawals and return flows on water availability. For 2010, the cumulative consumptive use at the juncture of the Wolf Creek RCA was estimated to be $25 \mathrm{Mgal} / \mathrm{d}$; Cordell Hull, $31 \mathrm{Mgal} / \mathrm{d}$; Old Hickory, $76 \mathrm{Mgal} / \mathrm{d}$; Cheatham, $83 \mathrm{Mgal} / \mathrm{d}$; and Barkley, $85 \mathrm{Mgal} / \mathrm{d}$ (table 1).

By category, estimated withdrawals and consumptive use for 2010 can be compared as follows. Thermoelectric power water withdrawals were more than eight times larger than public-supply water withdrawals (fig. 7). Industrial water withdrawals were slightly more than two times the irrigation water withdrawals. Consumptive use accounted for 2.5 percent of the total withdrawals. Consumptive use for industry and public supply was $72.5 \mathrm{Mgal} / \mathrm{d}$, and together these categories accounted for 84 percent of the total consumptive use. Consumptive use for irrigation was $13.6 \mathrm{Mgal} / \mathrm{d}$ and accounted for 16 percent of the total consumptive use. 
Table 3. Total water use by category, reservoir catchment area, hydrologic unit, and county, Cumberland River watershed, 2010.

[Values may not add to totals because of independent rounding. All values in million gallons per day; --, no RCA associated with withdrawal; RCA, reservoir catchment area; $<$, less than; $<0.001$, actual values less than 0.001 not in table but used to sum totals. Areal extent of all reservoir catchment areas is less than the areal extent of the Cumberland River watershed]

\begin{tabular}{|c|c|c|c|c|c|c|c|c|c|c|c|c|}
\hline \multirow{3}{*}{$\begin{array}{l}\text { RCA } \\
\text { Hydrologic unit } \\
\text { County, State } \\
\text { (See figs. } 3 \text { and 4) }\end{array}$} & \multicolumn{11}{|c|}{ Water withdrawals } & \multirow{3}{*}{$\begin{array}{c}\text { Total } \\
\text { return } \\
\text { flow }\end{array}$} \\
\hline & \multicolumn{3}{|c|}{ Public supply } & \multicolumn{3}{|c|}{ Industrial } & \multicolumn{3}{|c|}{ Irrigation } & \multicolumn{2}{|c|}{ Thermoelectric } & \\
\hline & $\begin{array}{c}\text { Surface } \\
\text { water }\end{array}$ & $\begin{array}{c}\text { Ground- } \\
\text { water }\end{array}$ & Total & $\begin{array}{c}\text { Surface } \\
\text { water }\end{array}$ & $\begin{array}{c}\text { Ground- } \\
\text { water }\end{array}$ & Total & $\begin{array}{c}\text { Surface } \\
\text { water }\end{array}$ & $\begin{array}{l}\text { Ground- } \\
\text { water }\end{array}$ & Total & $\begin{array}{c}\text { Surface } \\
\text { water }\end{array}$ & $\begin{array}{c}\text { Ground- } \\
\text { water }\end{array}$ & \\
\hline \multicolumn{13}{|l|}{ Martins Fork } \\
\hline \multicolumn{13}{|l|}{ Upper Cumberland 05130101} \\
\hline Bell County, Kentucky & 0.00 & 0.00 & 0.00 & 0.00 & 0.00 & 0.00 & $<0.001$ & 0.00 & $<0.001$ & 0.00 & 0.00 & 0.00 \\
\hline Harlan County, Kentucky & 2.30 & 0.00 & 2.30 & 0.00 & 0.00 & 0.00 & 0.00 & 0.00 & 0.00 & 0.00 & 0.00 & 0.00 \\
\hline Upper Cumberland total & 2.30 & 0.00 & 2.30 & 0.00 & 0.00 & 0.00 & $<0.001$ & 0.00 & $<0.001$ & 0.00 & 0.00 & 0.00 \\
\hline Martins Fork RCA total & 2.30 & 0.00 & 2.30 & 0.00 & $\mathbf{0 . 0 0}$ & 0.00 & $<0.001$ & 0.00 & $<0.001$ & $\mathbf{0 . 0 0}$ & $\mathbf{0 . 0 0}$ & 0.00 \\
\hline
\end{tabular}

Laurel

Upper Cumberland 05130101

\begin{tabular}{|c|c|c|c|c|c|c|c|c|c|c|c|c|}
\hline Knox County, Kentucky & 0.00 & 0.00 & 0.00 & 0.00 & 0.00 & 0.00 & 0.00 & 0.00 & 0.00 & 0.00 & 0.00 & 0.00 \\
\hline Laurel County, Kentucky & 5.01 & 0.00 & 5.01 & 0.00 & 0.00 & 0.00 & 0.009 & 0.00 & 0.009 & 0.00 & 0.00 & 5.16 \\
\hline Whitley County, Kentucky & 3.82 & 0.00 & 3.82 & 0.00 & 0.00 & 0.00 & 0.00 & 0.00 & 0.00 & 0.00 & 0.00 & 4.52 \\
\hline Upper Cumberland total & 8.83 & 0.00 & 8.83 & 0.00 & 0.00 & 0.00 & 0.009 & 0.00 & 0.009 & 0.00 & 0.00 & 9.68 \\
\hline Laurel RCA total & 8.83 & 0.00 & 8.83 & 0.00 & 0.00 & 0.00 & 0.009 & 0.00 & 0.009 & 0.00 & 0.00 & 9.68 \\
\hline
\end{tabular}

\section{Wolf Creek}

Upper Cumberland 05130101

\begin{tabular}{|c|c|c|c|c|c|c|c|c|c|c|c|c|}
\hline Bell County, Kentucky & 3.84 & 0.00 & 3.84 & 0.00 & 0.00 & 0.00 & 0.029 & 0.00 & 0.029 & 0.00 & 0.00 & 4.51 \\
\hline Clay County, Kentucky & 0.00 & 0.00 & 0.00 & 0.00 & 0.00 & 0.00 & $<0.001$ & 0.00 & $<0.001$ & 0.00 & 0.00 & 0.00 \\
\hline Harlan County, Kentucky & 0.00 & 1.36 & 1.36 & 0.00 & 0.00 & 0.00 & 0.00 & 0.00 & 0.00 & 0.00 & 0.00 & 2.76 \\
\hline Knox County, Kentucky & 0.326 & 0.00 & 0.326 & 0.00 & 0.00 & 0.00 & 0.00 & 0.00 & 0.00 & 0.00 & 0.00 & 1.03 \\
\hline Laurel County, Kentucky & 0.00 & 0.00 & 0.00 & 0.00 & 0.00 & 0.00 & $<0.001$ & 0.00 & $<0.001$ & 0.00 & 0.00 & 0.045 \\
\hline Leslie County, Kentucky & 0.00 & 0.00 & 0.00 & 0.00 & 0.00 & 0.00 & 0.00 & 0.00 & 0.00 & 0.00 & 0.00 & 0.00 \\
\hline Letcher County, Kentucky & 0.00 & 0.00 & 0.00 & 0.00 & 0.00 & 0.00 & 0.00 & 0.00 & 0.00 & 0.00 & 0.00 & 0.033 \\
\hline McCreary County, Kentucky & 1.17 & 0.00 & 1.17 & 0.00 & 0.00 & 0.00 & 0.017 & 0.00 & 0.017 & 0.00 & 0.00 & 0.906 \\
\hline Pulaski County, Kentucky & 0.00 & 0.00 & 0.00 & 0.00 & 0.00 & 0.00 & 0.00 & 0.00 & 0.00 & 0.00 & 0.00 & 0.060 \\
\hline Whitley County, Kentucky & 1.55 & 0.00 & 1.55 & 0.00 & 0.00 & 0.00 & 0.00 & 0.00 & 0.00 & 0.00 & 0.00 & 2.21 \\
\hline Campbell County, Tennessee & 0.954 & 0.00 & 0.954 & 0.00 & 0.00 & 0.00 & 0.00 & 0.021 & 0.021 & 0.00 & 0.00 & 0.00 \\
\hline Claiborne County, Tennessee & 0.00 & 0.151 & 0.151 & 0.00 & 0.00 & 0.00 & 0.00 & 0.005 & 0.005 & 0.00 & 0.00 & 0.00 \\
\hline Scott County, Tennessee & 0.00 & 0.00 & 0.00 & 0.00 & 0.00 & 0.00 & 0.00 & 0.00 & 0.00 & 0.00 & 0.00 & 0.00 \\
\hline pper Cumberland total & 7.83 & 1.51 & 9.34 & 0.00 & 0.00 & 0.00 & 0.046 & 0.026 & 0.072 & 0.00 & 0.00 & 11.6 \\
\hline
\end{tabular}


Table 3. Total water use by category, reservoir catchment area, hydrologic unit, and county, Cumberland River watershed, 2010.—Continued

[Values may not add to totals because of independent rounding. All values in million gallons per day; --, no RCA associated with withdrawal; RCA, reservoir catchment area; $<$, less than; $<0.001$, actual values less than 0.001 not in table but used to sum totals. Areal extent of all reservoir catchment areas is less than the areal extent of the Cumberland River watershed]

\begin{tabular}{|c|c|c|c|c|c|c|c|c|c|c|c|c|}
\hline \multirow{3}{*}{$\begin{array}{l}\text { RCA } \\
\text { Hydrologic unit } \\
\text { County, State } \\
\text { (See figs. } 3 \text { and 4) }\end{array}$} & \multicolumn{11}{|c|}{ Water withdrawals } & \multirow{3}{*}{$\begin{array}{c}\text { Total } \\
\text { return } \\
\text { flow }\end{array}$} \\
\hline & \multicolumn{3}{|c|}{ Public supply } & \multicolumn{3}{|c|}{ Industrial } & \multicolumn{3}{|c|}{ Irrigation } & \multicolumn{2}{|c|}{ Thermoelectric } & \\
\hline & $\begin{array}{c}\text { Surface } \\
\text { water }\end{array}$ & $\begin{array}{c}\text { Ground- } \\
\text { water }\end{array}$ & Total & $\begin{array}{c}\text { Surface } \\
\text { water }\end{array}$ & $\begin{array}{c}\text { Ground- } \\
\text { water }\end{array}$ & Total & $\begin{array}{c}\text { Surface } \\
\text { water }\end{array}$ & $\begin{array}{c}\text { Ground- } \\
\text { water }\end{array}$ & Total & $\begin{array}{c}\text { Surface } \\
\text { water }\end{array}$ & $\begin{array}{c}\text { Ground- } \\
\text { water }\end{array}$ & \\
\hline \multicolumn{13}{|l|}{ Rockcastle 05130102} \\
\hline Clay County, Kentucky & 0.00 & 0.00 & 0.00 & 0.00 & 0.00 & 0.00 & $<0.001$ & 0.00 & $<0.001$ & 0.00 & 0.00 & 0.008 \\
\hline Jackson County, Kentucky & 1.80 & 0.00 & 1.80 & 0.00 & 0.00 & 0.00 & 0.006 & 0.00 & 0.006 & 0.00 & 0.00 & 0.510 \\
\hline Laurel County, Kentucky & 4.09 & 0.00 & 4.09 & 0.00 & 0.00 & 0.00 & 0.011 & 0.00 & 0.011 & 0.00 & 0.00 & 0.045 \\
\hline Madison County, Kentucky & 0.00 & 0.00 & 0.00 & 0.00 & 0.00 & 0.00 & $<0.001$ & $<0.001$ & $<0.001$ & 0.00 & 0.00 & 0.00 \\
\hline Pulaski County, Kentucky & 0.00 & 0.00 & 0.00 & 0.00 & 0.00 & 0.00 & 0.019 & 0.00 & 0.019 & 0.00 & 0.00 & 0.00 \\
\hline Rockcastle County, Kentucky & 2.34 & 0.00 & 2.34 & 0.00 & 0.00 & 0.00 & 0.008 & 0.00 & 0.008 & 0.00 & 0.00 & 0.498 \\
\hline Rockcastle total & 8.23 & 0.00 & 8.23 & 0.00 & 0.00 & 0.00 & 0.044 & 0.00 & 0.044 & 0.00 & 0.00 & 1.06 \\
\hline \multicolumn{13}{|l|}{ Lake Cumberland 05130103} \\
\hline Casey County, Kentucky & 0.00 & 0.00 & 0.00 & 0.00 & 0.00 & 0.00 & 0.005 & 0.00 & 0.005 & 0.00 & 0.00 & 0.00 \\
\hline Clinton County, Kentucky & 3.40 & 0.00 & 3.40 & 0.00 & 0.00 & 0.00 & 0.003 & 0.00 & 0.003 & 0.00 & 0.00 & 0.00 \\
\hline Laurel County, Kentucky & 0.00 & 0.00 & 0.00 & 0.00 & 0.00 & 0.00 & $<0.001$ & 0.00 & $<0.001$ & 0.00 & 0.00 & 0.00 \\
\hline Lincoln County, Kentucky & 0.277 & 0.00 & 0.277 & 0.00 & 0.00 & 0.00 & 0.018 & 0.00 & 0.018 & 0.00 & 0.00 & 0.006 \\
\hline McCreary County, Kentucky & 0.00 & 0.00 & 0.00 & 0.00 & 0.00 & 0.00 & 0.003 & 0.00 & 0.003 & 0.00 & 0.00 & 0.00 \\
\hline Pulaski County, Kentucky & 9.04 & 0.00 & 9.04 & 0.00 & 0.00 & 0.00 & 0.200 & 0.00 & 0.200 & 96.4 & 0.00 & 101 \\
\hline Rockcastle County, Kentucky & 0.00 & 0.00 & 0.00 & 0.00 & 0.00 & 0.00 & 0.001 & 0.00 & 0.001 & 0.00 & 0.00 & 0.069 \\
\hline Russell County, Kentucky & 3.48 & 0.00 & 3.48 & 0.00 & 0.00 & 0.00 & 0.051 & 0.00 & 0.051 & 0.00 & 0.00 & 0.171 \\
\hline Wayne County, Kentucky & 2.46 & 0.00 & 2.46 & 0.00 & 0.00 & 0.00 & 0.013 & 0.00 & 0.013 & 0.00 & 0.00 & 0.743 \\
\hline Pickett County, Tennessee & 0.00 & 0.00 & 0.00 & 0.00 & 0.00 & 0.00 & 0.00 & 0.00 & 0.00 & 0.00 & 0.00 & 0.00 \\
\hline Lake Cumberland total & 18.7 & 0.00 & 18.7 & 0.00 & 0.00 & 0.00 & 0.294 & 0.00 & 0.294 & 96.4 & 0.00 & 102.06 \\
\hline
\end{tabular}


Table 3. Total water use by category, reservoir catchment area, hydrologic unit, and county, Cumberland River watershed, 2010.-Continued

[Values may not add to totals because of independent rounding. All values in million gallons per day; --, no RCA associated with withdrawal; RCA, reservoir catchment area; $<$, less than; $<0.001$, actual values less than 0.001 not in table but used to sum totals. Areal extent of all reservoir catchment areas is less than the areal extent of the Cumberland River watershed]

\begin{tabular}{|c|c|c|c|c|c|c|c|c|c|c|c|c|}
\hline \multirow{3}{*}{$\begin{array}{l}\text { RCA } \\
\text { Hydrologic unit } \\
\text { County, State } \\
\text { (See figs. } 3 \text { and 4) }\end{array}$} & \multicolumn{11}{|c|}{ Water withdrawals } & \multirow{3}{*}{$\begin{array}{c}\text { Total } \\
\text { return } \\
\text { flow }\end{array}$} \\
\hline & \multicolumn{3}{|c|}{ Public supply } & \multicolumn{3}{|c|}{ Industrial } & \multicolumn{3}{|c|}{ Irrigation } & \multicolumn{2}{|c|}{ Thermoelectric } & \\
\hline & $\begin{array}{c}\text { Surface } \\
\text { water }\end{array}$ & $\begin{array}{c}\text { Ground- } \\
\text { water }\end{array}$ & Total & $\begin{array}{c}\text { Surface } \\
\text { water }\end{array}$ & $\begin{array}{c}\text { Ground- } \\
\text { water }\end{array}$ & Total & $\begin{array}{c}\text { Surface } \\
\text { water }\end{array}$ & $\begin{array}{c}\text { Ground- } \\
\text { water }\end{array}$ & Total & $\begin{array}{c}\text { Surface } \\
\text { water }\end{array}$ & $\begin{array}{c}\text { Ground- } \\
\text { water }\end{array}$ & \\
\hline \multicolumn{13}{|l|}{ South Fork Cumberland 05130104} \\
\hline McCreary County, Kentucky & 0.722 & 0.00 & 0.722 & 0.00 & 0.00 & 0.00 & 0.020 & 0.00 & 0.020 & 0.00 & 0.00 & 0.00 \\
\hline Pulaski County, Kentucky & 0.054 & 0.00 & 0.054 & 0.240 & 0.00 & 0.240 & 0.011 & 0.00 & 0.011 & 0.00 & 0.00 & 0.003 \\
\hline Wayne County, Kentucky & 0.00 & 0.00 & 0.00 & 0.00 & 0.00 & 0.00 & 0.006 & 0.00 & 0.006 & 0.00 & 0.00 & 0.00 \\
\hline Anderson County, Tennessee & 0.00 & 0.00 & 0.00 & 0.00 & 0.00 & 0.00 & 0.002 & 0.009 & 0.011 & 0.00 & 0.00 & 0.00 \\
\hline Campbell County, Tennessee & 0.00 & 0.00 & 0.00 & 0.00 & 0.00 & 0.00 & 0.00 & 0.007 & 0.007 & 0.00 & 0.00 & 0.00 \\
\hline Fentress County, Tennessee & 1.74 & 0.00 & 1.74 & 0.00 & 0.00 & 0.00 & 0.00 & 0.009 & 0.009 & 0.00 & 0.00 & 0.00 \\
\hline Morgan County, Tennessee & 0.00 & 0.00 & 0.00 & 0.00 & 0.00 & 0.00 & 0.00 & 0.00 & 0.00 & 0.00 & 0.00 & 0.00 \\
\hline Pickett County, Tennessee & 0.00 & 0.00 & 0.00 & 0.00 & 0.00 & 0.00 & 0.00 & 0.00 & 0.00 & 0.00 & 0.00 & 0.00 \\
\hline Scott County, Tennessee & 2.58 & 0.00 & 2.58 & 0.00 & 0.00 & 0.00 & 0.00 & 0.00 & 0.00 & 0.00 & 0.00 & 0.00 \\
\hline South Fork Cumberland total & 5.09 & 0.00 & 5.09 & 0.240 & 0.00 & 0.240 & 0.040 & 0.025 & 0.064 & 0.00 & 0.00 & 0.003 \\
\hline Wolf Creek RCA total & 39.8 & 1.51 & 41.3 & 0.240 & 0.00 & 0.240 & 0.423 & 0.051 & 0.474 & 96.4 & 0.00 & 114.69 \\
\hline \multicolumn{13}{|l|}{ Dale Hollow } \\
\hline \multicolumn{13}{|l|}{ Obey 05130105} \\
\hline Clinton County, Kentucky & 0.00 & 0.00 & 0.00 & 0.00 & 0.00 & 0.00 & 0.006 & 0.00 & 0.006 & 0.00 & 0.00 & 0.530 \\
\hline Cumberland County, Kentucky & 0.00 & 0.00 & 0.00 & 0.00 & 0.00 & 0.00 & 0.001 & 0.00 & 0.001 & 0.00 & 0.00 & 0.027 \\
\hline Wayne County, Kentucky & 0.00 & 0.00 & 0.00 & 0.00 & 0.00 & 0.00 & $<0.001$ & 0.00 & $<0.001$ & 0.00 & 0.00 & 0.00 \\
\hline Clay County, Tennessee & 0.00 & 0.00 & 0.00 & 0.00 & 0.00 & 0.00 & 0.00 & 0.006 & 0.006 & 0.00 & 0.00 & 0.00 \\
\hline Cumberland County, Tennessee & 0.00 & 0.00 & 0.00 & 0.00 & 0.00 & 0.00 & 0.00 & 0.005 & 0.005 & 0.00 & 0.00 & 0.026 \\
\hline Fentress County, Tennessee & 0.00 & 0.00 & 0.00 & 0.00 & 0.00 & 0.00 & 0.00 & 0.010 & 0.010 & 0.00 & 0.00 & 0.052 \\
\hline Overton County, Tennessee & 0.00 & 0.00 & 0.00 & 0.00 & 0.00 & 0.00 & 0.00 & 0.064 & 0.064 & 0.00 & 0.00 & 0.00 \\
\hline Pickett County, Tennessee & 0.620 & 0.00 & 0.620 & 0.00 & 0.00 & 0.00 & 0.00 & 0.00 & 0.00 & 0.00 & 0.00 & 0.00 \\
\hline Putnam County, Tennessee & 1.27 & 0.008 & 1.28 & 0.00 & 0.00 & 0.00 & 0.00 & 0.006 & 0.006 & 0.00 & 0.00 & 0.00 \\
\hline Obey total & 1.89 & 0.008 & 1.90 & 0.00 & 0.00 & 0.00 & 0.007 & 0.092 & 0.099 & 0.00 & 0.00 & 0.635 \\
\hline Dale Hollow RCA total & 1.89 & 0.008 & 1.90 & 0.00 & $\mathbf{0 . 0 0}$ & 0.00 & 0.007 & 0.092 & 0.099 & 0.00 & 0.00 & 0.635 \\
\hline
\end{tabular}


Table 3. Total water use by category, reservoir catchment area, hydrologic unit, and county, Cumberland River watershed, 2010. —Continued

[Values may not add to totals because of independent rounding. All values in million gallons per day; --, no RCA associated with withdrawal; RCA, reservoir catchment area; $<$, less than; $<0.001$, actual values less than 0.001 not in table but used to sum totals. Areal extent of all reservoir catchment areas is less than the areal extent of the Cumberland River watershed]

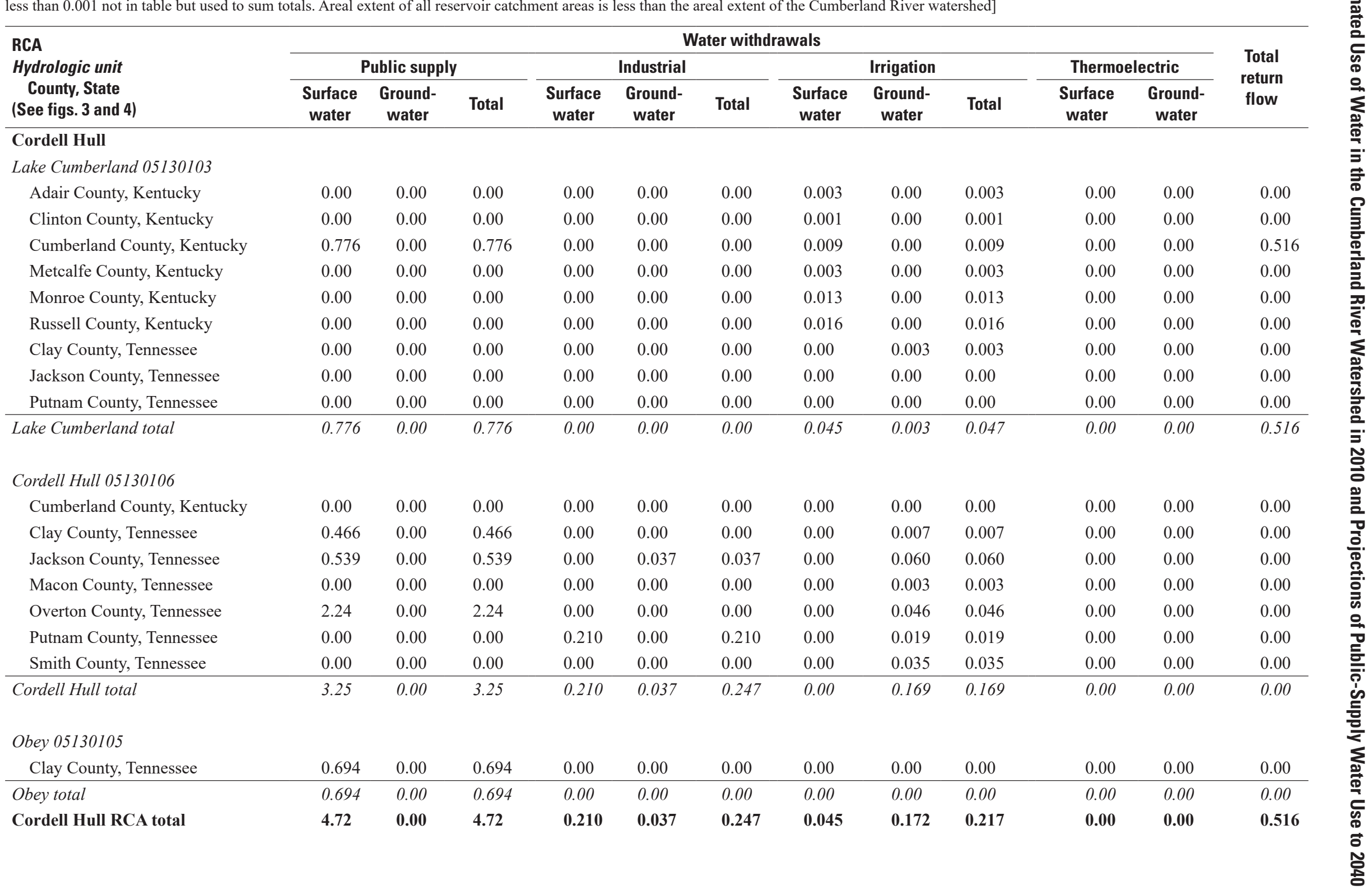


Table 3. Total water use by category, reservoir catchment area, hydrologic unit, and county, Cumberland River watershed, 2010.-Continued

[Values may not add to totals because of independent rounding. All values in million gallons per day; --, no RCA associated with withdrawal; RCA, reservoir catchment area; $<$, less than; $<0.001$, actual values less than 0.001 not in table but used to sum totals. Areal extent of all reservoir catchment areas is less than the areal extent of the Cumberland River watershed]

\begin{tabular}{|c|c|c|c|c|c|c|c|c|c|c|c|c|}
\hline \multirow{3}{*}{$\begin{array}{l}\text { RCA } \\
\text { Hydrologic unit } \\
\text { County, State } \\
\text { (See figs. } 3 \text { and 4) }\end{array}$} & \multicolumn{11}{|c|}{ Water withdrawals } & \multirow{3}{*}{$\begin{array}{l}\text { Total } \\
\text { return } \\
\text { flow }\end{array}$} \\
\hline & \multicolumn{3}{|c|}{ Public supply } & \multicolumn{3}{|c|}{ Industrial } & \multicolumn{3}{|c|}{ Irrigation } & \multicolumn{2}{|c|}{ Thermoelectric } & \\
\hline & $\begin{array}{c}\text { Surface } \\
\text { water }\end{array}$ & $\begin{array}{c}\text { Ground- } \\
\text { water }\end{array}$ & Total & $\begin{array}{c}\text { Surface } \\
\text { water }\end{array}$ & $\begin{array}{c}\text { Ground- } \\
\text { water }\end{array}$ & Total & $\begin{array}{c}\text { Surface } \\
\text { water }\end{array}$ & $\begin{array}{c}\text { Ground- } \\
\text { water }\end{array}$ & Total & $\begin{array}{c}\text { Surface } \\
\text { water }\end{array}$ & $\begin{array}{c}\text { Ground- } \\
\text { water }\end{array}$ & \\
\hline \multicolumn{13}{|l|}{ Center Hill } \\
\hline \multicolumn{13}{|l|}{ Collins 05130107} \\
\hline Cannon County, Tennessee & 0.00 & 0.00 & 0.00 & 0.00 & 0.00 & 0.00 & 0.00 & 0.021 & 0.021 & 0.00 & 0.00 & 0.00 \\
\hline Coffee County, Tennessee & 0.00 & 0.00 & 0.00 & 0.00 & 0.00 & 0.00 & 0.021 & 0.178 & 0.199 & 0.00 & 0.00 & 0.00 \\
\hline DeKalb County, Tennessee & 0.00 & 0.00 & 0.00 & 0.00 & 0.00 & 0.00 & 0.00 & 0.00 & 0.00 & 0.00 & 0.00 & 0.00 \\
\hline Grundy County, Tennessee & 0.862 & 0.00 & 0.862 & 0.00 & 0.00 & 0.00 & 0.00 & 0.283 & 0.283 & 0.00 & 0.00 & 0.00 \\
\hline Sequatchie County, Tennessee & 0.00 & 0.00 & 0.00 & 0.00 & 0.00 & 0.00 & 0.00 & 0.001 & 0.001 & 0.00 & 0.00 & 0.00 \\
\hline Van Buren County, Tennessee & 0.00 & 0.00 & 0.00 & 0.00 & 0.00 & 0.00 & 0.00 & 0.00 & 0.00 & 0.00 & 0.00 & 0.00 \\
\hline Warren County, Tennessee & 5.10 & 0.00 & 5.10 & 0.00 & 0.00 & 0.00 & 0.680 & 3.73 & 4.41 & 0.00 & 0.00 & 5.31 \\
\hline Collins total & 5.96 & 0.00 & 5.96 & 0.00 & 0.00 & 0.00 & 0.701 & 4.21 & 4.91 & 0.00 & 0.00 & 5.31 \\
\hline \multicolumn{13}{|l|}{ Caney 05130108} \\
\hline Bledsoe County, Tennessee & 0.729 & 0.00 & 0.729 & 0.00 & 0.00 & 0.00 & 0.00 & 0.139 & 0.139 & 0.00 & 0.00 & 0.280 \\
\hline Cannon County, Tennessee & 0.00 & 0.00 & 0.00 & 0.00 & 0.00 & 0.00 & 0.00 & $<0.001$ & $<0.001$ & 0.00 & 0.00 & 0.00 \\
\hline Cumberland County, Tennessee & 0.00 & 0.00 & 0.00 & 0.00 & 0.00 & 0.00 & 0.00 & 0.058 & 0.058 & 0.00 & 0.00 & 0.044 \\
\hline DeKalb County, Tennessee & 0.00 & 0.00 & 0.00 & 0.00 & 0.00 & 0.00 & 0.00 & 0.00 & 0.00 & 0.00 & 0.00 & 2.21 \\
\hline Putnam County, Tennessee & 13.3 & 0.00 & 13.3 & 0.00 & 0.00 & 0.00 & 0.00 & 0.038 & 0.038 & 0.00 & 0.00 & 1.51 \\
\hline Sequatchie County, Tennessee & 0.00 & 0.00 & 0.00 & 0.00 & 0.00 & 0.00 & 0.00 & $<0.001$ & $<0.001$ & 0.00 & 0.00 & 0.00 \\
\hline Van Buren County, Tennessee & 0.59 & 0.00 & 0.59 & 0.00 & 0.00 & 0.00 & 0.00 & 0.00 & 0.00 & 0.00 & 0.00 & 0.170 \\
\hline Warren County, Tennessee & 0.00 & 0.00 & 0.00 & 0.00 & 0.00 & 0.00 & 0.070 & 0.384 & 0.454 & 0.00 & 0.00 & 0.020 \\
\hline White County, Tennessee & 3.54 & 0.00 & 3.54 & 0.00 & 0.00 & 0.00 & 0.00 & 0.040 & 0.040 & 0.00 & 0.00 & 1.60 \\
\hline Caney total & 18.1 & 0.00 & 18.1 & 0.00 & 0.00 & 0.00 & 0.070 & 0.660 & 0.730 & 0.00 & 0.00 & 5.83 \\
\hline Center Hill RCA total & 24.1 & 0.00 & 24.1 & 0.00 & 0.00 & 0.00 & 0.771 & 4.87 & 5.64 & 0.00 & 0.00 & 11.1 \\
\hline \multicolumn{13}{|l|}{ Old Hickory } \\
\hline \multicolumn{13}{|l|}{ Cordell Hull 05130106} \\
\hline Smith County, Tennessee & 0.00 & 0.00 & 0.00 & 0.00 & 0.00 & 0.00 & 0.00 & 0.003 & 0.003 & 0.00 & 0.00 & 0.00 \\
\hline Cordell Hull total & 0.00 & 0.00 & 0.00 & 0.00 & 0.00 & 0.00 & 0.00 & 0.003 & 0.003 & 0.00 & 0.00 & 0.00 \\
\hline
\end{tabular}


Table 3. Total water use by category, reservoir catchment area, hydrologic unit, and county, Cumberland River watershed, 2010.-Continued

[Values may not add to totals because of independent rounding. All values in million gallons per day; --, no RCA associated with withdrawal; RCA, reservoir catchment area; $<$, less than; $<0.001$, actual values less than 0.001 not in table but used to sum totals. Areal extent of all reservoir catchment areas is less than the areal extent of the Cumberland River watershed]

\begin{tabular}{|c|c|c|c|c|c|c|c|c|c|c|c|c|}
\hline \multirow{3}{*}{$\begin{array}{l}\text { RCA } \\
\text { Hydrologic unit } \\
\text { County, State } \\
\text { (See figs. } 3 \text { and 4) }\end{array}$} & \multicolumn{11}{|c|}{ Water withdrawals } & \multirow{3}{*}{$\begin{array}{c}\text { Total } \\
\text { return } \\
\text { flow }\end{array}$} \\
\hline & \multicolumn{3}{|c|}{ Public supply } & \multicolumn{3}{|c|}{ Industrial } & \multicolumn{3}{|c|}{ Irrigation } & \multicolumn{2}{|c|}{ Thermoelectric } & \\
\hline & $\begin{array}{c}\text { Surface } \\
\text { water }\end{array}$ & $\begin{array}{c}\text { Ground- } \\
\text { water }\end{array}$ & Total & $\begin{array}{c}\text { Surface } \\
\text { water }\end{array}$ & $\begin{array}{c}\text { Ground- } \\
\text { water }\end{array}$ & Total & $\begin{array}{c}\text { Surface } \\
\text { water }\end{array}$ & $\begin{array}{c}\text { Ground- } \\
\text { water }\end{array}$ & Total & $\begin{array}{c}\text { Surface } \\
\text { water }\end{array}$ & $\begin{array}{c}\text { Ground- } \\
\text { water }\end{array}$ & \\
\hline \multicolumn{13}{|l|}{ Caney 05130108} \\
\hline Cannon County, Tennessee & 0.00 & 0.00 & 0.00 & 0.00 & 0.00 & 0.00 & 0.00 & 0.020 & 0.020 & 0.00 & 0.00 & 0.00 \\
\hline DeKalb County, Tennessee & 1.81 & 0.073 & 1.89 & 0.00 & 0.00 & 0.00 & 0.00 & 0.00 & 0.00 & 0.00 & 0.00 & 0.00 \\
\hline Putnam County, Tennessee & 0.00 & 0.00 & 0.00 & 0.00 & 0.00 & 0.00 & 0.00 & 0.007 & 0.007 & 0.00 & 0.00 & 0.00 \\
\hline Rutherford County, Tennessee & 0.00 & 0.00 & 0.00 & 0.00 & 0.00 & 0.00 & 0.00 & $<0.001$ & $<0.001$ & 0.00 & 0.00 & 0.00 \\
\hline Smith County, Tennessee & 0.00 & 0.00 & 0.00 & 0.00 & 0.00 & 0.00 & 0.00 & 0.059 & 0.059 & 0.00 & 0.00 & 0.00 \\
\hline Wilson County, Tennessee & 0.00 & 0.00 & 0.00 & 0.00 & 0.00 & 0.00 & 0.00 & 0.008 & 0.008 & 0.00 & 0.00 & 0.00 \\
\hline Caney total & 1.81 & 0.073 & 1.89 & 0.00 & 0.00 & 0.00 & 0.00 & 0.095 & 0.095 & 0.00 & 0.00 & 0.00 \\
\hline \multicolumn{13}{|l|}{ Old Hickory Lake 05130201} \\
\hline Davidson County, Tennessee & 0.834 & 0.00 & 0.834 & 16.9 & 0.00 & 16.9 & $<0.001$ & 0.003 & 0.003 & 0.00 & 0.00 & 29.1 \\
\hline Dekalb County, Tennessee & 0.00 & 0.00 & 0.00 & 0.00 & 0.00 & 0.00 & 0.00 & 0.00 & 0.00 & 0.00 & 0.00 & 1.16 \\
\hline Macon County, Tennessee & 0.00 & 0.00 & 0.00 & 0.00 & 0.00 & 0.00 & 0.00 & 0.016 & 0.016 & 0.00 & 0.00 & 0.00 \\
\hline Robertson County, Tennessee & 0.00 & 0.00 & 0.00 & 0.00 & 0.00 & 0.00 & 0.00 & $<0.001$ & $<0.001$ & 0.00 & 0.00 & 0.00 \\
\hline Smith County, Tennessee & 2.09 & 0.00 & 2.09 & 0.00 & 0.00 & 0.00 & 0.00 & 0.063 & 0.063 & 0.00 & 0.00 & 2.81 \\
\hline Sumner County, Tennessee & 23.8 & 0.00 & 23.8 & 1.01 & 0.00 & 1.01 & 0.00 & 0.110 & 0.110 & 559.20 & 0.00 & 561.71 \\
\hline Trousdale County, Tennessee & 0.922 & 0.00 & 0.922 & 0.00 & 0.00 & 0.00 & 0.00 & 0.090 & 0.090 & 0.00 & 0.00 & 0.00 \\
\hline Wilson County, Tennessee & 13.5 & 0.211 & 13.7 & 0.402 & 0.00 & 0.402 & 0.00 & 0.048 & 0.048 & 0.00 & 0.00 & 0.00 \\
\hline Old Hickory Lake total & 41.2 & 0.211 & 41.4 & 18.3 & 0.00 & 18.3 & $<0.001$ & 0.331 & 0.331 & 559.20 & 0.00 & 594.78 \\
\hline Old Hickory RCA total & 43.0 & 0.283 & 43.3 & 18.3 & 0.00 & 18.3 & $<0.001$ & 0.429 & 0.430 & 559.20 & 0.00 & 594.78 \\
\hline \multicolumn{13}{|l|}{ J. Percy Priest } \\
\hline \multicolumn{13}{|l|}{ Stones 05130203} \\
\hline Cannon County, Tennessee & 1.14 & 0.00 & 1.14 & 0.00 & 0.00 & 0.00 & 0.00 & 0.039 & 0.039 & 0.00 & 0.00 & 0.00 \\
\hline Coffee County, Tennessee & 0.00 & 0.00 & 0.00 & 0.00 & 0.00 & 0.00 & 0.0002 & 0.001 & 0.002 & 0.00 & 0.00 & 0.00 \\
\hline Davidson County, Tennessee & 0.00 & 0.00 & 0.00 & 0.00 & 0.00 & 0.00 & 0.003 & 0.029 & 0.032 & 0.00 & 0.00 & 0.382 \\
\hline Putnam County, Tennessee & 0.00 & 0.00 & 0.00 & 0.00 & 0.00 & 0.00 & 0.00 & 0.00 & 0.00 & 0.00 & 0.00 & 0.600 \\
\hline Rutherford County, Tennessee & 33.8 & 0.00 & 33.8 & 0.469 & 0.016 & 0.485 & 0.00 & 0.881 & 0.881 & 0.00 & 0.00 & 15.8 \\
\hline Williamson County, Tennessee & 0.00 & 0.00 & 0.00 & 0.00 & 0.00 & 0.00 & $<0.001$ & 0.001 & 0.001 & 0.00 & 0.00 & 0.00 \\
\hline Wilson County, Tennessee & 0.00 & 1.71 & 1.71 & 0.00 & 0.00 & 0.00 & 0.00 & 0.021 & 0.021 & 0.00 & 0.00 & 0.00 \\
\hline Stones total & 34.9 & 1.71 & 36.6 & 0.469 & 0.016 & 0.485 & 0.003 & 0.973 & 0.976 & 0.00 & 0.00 & 16.7 \\
\hline J. Percy Priest RCA total & 34.9 & 1.71 & 36.6 & 0.469 & 0.016 & 0.485 & 0.003 & 0.973 & 0.976 & 0.00 & 0.00 & 16.7 \\
\hline
\end{tabular}


Table 3. Total water use by category, reservoir catchment area, hydrologic unit, and county, Cumberland River watershed, 2010.—Continued

[Values may not add to totals because of independent rounding. All values in million gallons per day; --, no RCA associated with withdrawal; RCA, reservoir catchment area; $<$, less than; $<0.001$, actual values less than 0.001 not in table but used to sum totals. Areal extent of all reservoir catchment areas is less than the areal extent of the Cumberland River watershed]

\begin{tabular}{|c|c|c|c|c|c|c|c|c|c|c|c|c|}
\hline \multirow{3}{*}{$\begin{array}{l}\text { RCA } \\
\text { Hydrologic unit } \\
\text { County, State } \\
\text { (See figs. } 3 \text { and 4) }\end{array}$} & \multicolumn{11}{|c|}{ Water withdrawals } & \multirow{3}{*}{$\begin{array}{c}\text { Total } \\
\text { return } \\
\text { flow }\end{array}$} \\
\hline & \multicolumn{3}{|c|}{ Public supply } & \multicolumn{3}{|c|}{ Industrial } & \multicolumn{3}{|c|}{ Irrigation } & \multicolumn{2}{|c|}{ Thermoelectric } & \\
\hline & $\begin{array}{c}\text { Surface } \\
\text { water }\end{array}$ & $\begin{array}{c}\text { Ground- } \\
\text { water }\end{array}$ & Total & $\begin{array}{c}\text { Surface } \\
\text { water }\end{array}$ & $\begin{array}{c}\text { Ground- } \\
\text { water }\end{array}$ & Total & $\begin{array}{c}\text { Surface } \\
\text { water }\end{array}$ & $\begin{array}{c}\text { Ground- } \\
\text { water }\end{array}$ & Total & $\begin{array}{c}\text { Surface } \\
\text { water }\end{array}$ & $\begin{array}{c}\text { Ground- } \\
\text { water }\end{array}$ & \\
\hline \multicolumn{13}{|l|}{ Cheatham } \\
\hline \multicolumn{13}{|l|}{ Sycamore 05130202} \\
\hline Cheatham County, Tennessee & 2.30 & 0.00 & 2.30 & 0.188 & 0.00 & 0.188 & 0.00 & 0.128 & 0.128 & 0.00 & 0.00 & 0.265 \\
\hline Davidson County, Tennessee & 135.54 & 0.00 & 135.54 & 8.32 & 0.436 & 8.76 & 0.022 & 0.220 & 0.242 & 0.00 & 0.00 & 163.62 \\
\hline Dickson County, Tennessee & 0.00 & 0.00 & 0.00 & 0.00 & 0.00 & 0.00 & $<0.001$ & $<0.001$ & $<0.001$ & 0.00 & 0.00 & 0.00 \\
\hline Robertson County, Tennessee & 0.00 & 0.00 & 0.00 & 0.00 & 0.00 & 0.00 & 0.00 & 0.128 & 0.128 & 0.00 & 0.00 & 0.00 \\
\hline Rutherford County, Tennessee & 0.00 & 0.00 & 0.00 & 0.00 & 0.00 & 0.00 & 0.00 & 0.001 & 0.001 & 0.00 & 0.00 & 0.00 \\
\hline Sumner County, Tennessee & 0.00 & 0.00 & 0.00 & 0.00 & 0.00 & 0.00 & 0.00 & 0.010 & 0.010 & 0.00 & 0.00 & 0.020 \\
\hline Williamson County, Tennessee & 0.00 & 0.00 & 0.00 & 0.00 & 0.00 & 0.00 & 0.006 & 0.031 & 0.037 & 0.00 & 0.00 & 0.00 \\
\hline Sycamore total & 137.85 & 0.00 & 137.85 & 8.51 & 0.436 & 8.94 & 0.028 & 0.519 & 0.547 & 0.00 & 0.00 & 163.90 \\
\hline \multicolumn{13}{|l|}{ Stones 05130203} \\
\hline Davidson County, Tennessee & 0.00 & 0.00 & 0.00 & 0.008 & 0.00 & 0.008 & 0.001 & 0.015 & 0.016 & 0.00 & 0.00 & 0.00 \\
\hline Wilson County, Tennessee & 0.00 & 0.00 & 0.00 & 0.00 & 0.00 & 0.00 & 0.00 & 0.002 & 0.002 & 0.00 & 0.00 & 0.00 \\
\hline Stones total & 0.00 & 0.00 & 0.00 & 0.008 & 0.00 & 0.008 & 0.001 & 0.017 & 0.019 & 0.00 & 0.00 & 0.00 \\
\hline \multicolumn{13}{|l|}{ Harpeth 05130204} \\
\hline Cheatham County, Tennessee & 0.652 & 0.00 & 0.652 & 0.339 & 0.00 & 0.339 & 0.00 & 0.073 & 0.073 & 0.00 & 0.00 & 1.11 \\
\hline Davidson County, Tennessee & 0.00 & 0.00 & 0.00 & 0.00 & 0.00 & 0.00 & 0.003 & 0.033 & 0.036 & 0.00 & 0.00 & 0.00 \\
\hline Dickson County, Tennessee & 3.67 & 0.00 & 3.67 & 0.00 & 0.00 & 0.00 & 0.025 & 0.050 & 0.075 & 0.00 & 0.00 & 3.22 \\
\hline Hickman County, Tennessee & 0.00 & 0.00 & 0.00 & 0.00 & 0.00 & 0.00 & 0.00 & 0.001 & 0.001 & 0.00 & 0.00 & 0.00 \\
\hline Rutherford County, Tennessee & 0.00 & 0.00 & 0.00 & 0.00 & 0.00 & 0.00 & 0.00 & 0.084 & 0.084 & 0.00 & 0.00 & 0.00 \\
\hline Williamson County, Tennessee & 1.69 & 0.112 & 1.80 & 0.00 & 0.00 & 0.00 & 0.078 & 0.396 & 0.474 & 0.00 & 0.00 & 0.680 \\
\hline Harpeth total & 6.01 & 0.112 & 6.12 & 0.339 & 0.00 & 0.339 & 0.106 & 0.635 & 0.741 & 0.00 & 0.00 & 5.01 \\
\hline Cheatham RCA total & 143.85 & 0.112 & 143.97 & 8.85 & 0.436 & 9.29 & 0.135 & 1.17 & 1.31 & 0.00 & 0.00 & 168.91 \\
\hline
\end{tabular}


[Values may not add to totals because of independent rounding. All values in million gallons per day; --, no RCA associated with withdrawal; RCA, reservoir catchment area; $<$, less than; $<0.001$, actual values less than 0.001 not in table but used to sum totals. Areal extent of all reservoir catchment areas is less than the areal extent of the Cumberland River watershed]

\begin{tabular}{|c|c|c|c|c|c|c|c|c|c|c|c|c|}
\hline \multirow{3}{*}{$\begin{array}{l}\text { RCA } \\
\text { Hydrologic unit } \\
\text { County, State } \\
\text { (See figs. } 3 \text { and 4) }\end{array}$} & \multicolumn{11}{|c|}{ Water withdrawals } & \multirow{3}{*}{$\begin{array}{c}\text { Total } \\
\text { return } \\
\text { flow }\end{array}$} \\
\hline & \multicolumn{3}{|c|}{ Public supply } & \multicolumn{3}{|c|}{ Industrial } & \multicolumn{3}{|c|}{ Irrigation } & \multicolumn{2}{|c|}{ Thermoelectric } & \\
\hline & $\begin{array}{c}\text { Surface } \\
\text { water }\end{array}$ & $\begin{array}{c}\text { Ground- } \\
\text { water }\end{array}$ & Total & $\begin{array}{c}\text { Surface } \\
\text { water }\end{array}$ & $\begin{array}{c}\text { Ground- } \\
\text { water }\end{array}$ & Total & $\begin{array}{c}\text { Surface } \\
\text { water }\end{array}$ & $\begin{array}{c}\text { Ground- } \\
\text { water }\end{array}$ & Total & $\begin{array}{c}\text { Surface } \\
\text { water }\end{array}$ & $\begin{array}{c}\text { Ground- } \\
\text { water }\end{array}$ & \\
\hline \multicolumn{13}{|l|}{ Barkley } \\
\hline \multicolumn{13}{|l|}{ Lower Cumberland 05130205} \\
\hline Caldwell County, Kentucky & 0.00 & 0.00 & 0.00 & 0.00 & 0.00 & 0.00 & 0.373 & 0.013 & 0.386 & 0.00 & 0.00 & 1.58 \\
\hline Christian County, Kentucky & 0.00 & 0.00 & 0.00 & 1.41 & 0.00 & 1.41 & 0.587 & 0.018 & 0.604 & 0.00 & 0.00 & 3.13 \\
\hline Crittenden County, Kentucky & 0.00 & 0.00 & 0.00 & 0.00 & 0.00 & 0.00 & 0.023 & 0.00 & 0.023 & 0.00 & 0.00 & 0.00 \\
\hline Livingston County, Kentucky & 0.00 & 0.00 & 0.00 & 0.00 & 0.00 & 0.00 & 0.030 & 0.00 & 0.030 & 0.00 & 0.00 & 0.00 \\
\hline Lyon County, Kentucky & 2.68 & 0.113 & 2.79 & 0.010 & 0.00 & 0.010 & 0.00 & 0.00 & 0.00 & 0.00 & 0.00 & 2.04 \\
\hline Todd County, Kentucky & 0.00 & 0.00 & 0.00 & 0.00 & 0.100 & 0.100 & 0.001 & $<0.001$ & 0.001 & 0.00 & 0.00 & 0.00 \\
\hline Trigg County, Kentucky & 14.9 & 0.00 & 14.9 & 0.00 & 0.00 & 0.00 & 0.634 & 0.009 & 0.643 & 0.00 & 0.00 & 0.681 \\
\hline Cheatham County, Tennessee & 0.00 & 0.00 & 0.00 & 0.00 & 0.00 & 0.00 & 0.00 & 0.048 & 0.048 & 0.00 & 0.00 & 0.247 \\
\hline Dickson County, Tennessee & 0.019 & 0.019 & 0.039 & 0.00 & 0.00 & 0.00 & 0.024 & 0.049 & 0.073 & 0.00 & 0.00 & 0.00 \\
\hline Houston County, Tennessee & 1.05 & 0.00 & 1.05 & 0.00 & 0.00 & 0.00 & 0.00 & 0.010 & 0.010 & 0.00 & 0.00 & 0.00 \\
\hline Montgomery County, Tennessee & 18.8 & 0.00 & 18.8 & 1.25 & 0.00 & 1.25 & 0.00 & 0.314 & 0.314 & 0.00 & 0.00 & 0.555 \\
\hline Robertson County, Tennessee & 0.00 & 0.00 & 0.00 & 0.00 & 0.00 & 0.00 & 0.00 & 0.002 & 0.002 & 0.00 & 0.00 & 0.00 \\
\hline Stewart County, Tennessee & 0.273 & 0.455 & 0.728 & 0.00 & 0.00 & 0.00 & 0.044 & 0.075 & 0.118 & $2,395.50$ & 0.00 & $2,418.60$ \\
\hline Lower Cumberland total & 37.7 & 0.587 & 38.3 & 2.67 & 0.100 & 2.77 & 1.71 & 0.538 & 2.25 & $2,395.50$ & 0.00 & $2,426.84$ \\
\hline \multicolumn{13}{|l|}{ Red 05130206} \\
\hline Christian County, Kentucky & 0.00 & 0.00 & 0.00 & 0.00 & 0.00 & 0.00 & 0.248 & 0.007 & 0.256 & 0.00 & 0.00 & 0.00 \\
\hline Logan County, Kentucky & 0.00 & 0.00 & 0.00 & 0.00 & 0.00 & 0.00 & 0.076 & 0.004 & 0.080 & 0.00 & 0.00 & 0.260 \\
\hline Simpson County, Kentucky & 0.00 & 0.00 & 0.00 & 0.00 & 0.00 & 0.00 & 0.013 & 0.00 & 0.013 & 0.00 & 0.00 & 0.00 \\
\hline Todd County, Kentucky & 3.88 & 0.00 & 3.88 & 0.00 & 0.00 & 0.00 & 0.211 & 0.006 & 0.217 & 0.00 & 0.00 & 0.593 \\
\hline Trigg County, Kentucky & 0.00 & 0.00 & 0.00 & 0.00 & 0.00 & 0.00 & 0.004 & $<0.001$ & 0.004 & 0.00 & 0.00 & 0.00 \\
\hline Cheatham County, Tennessee & 0.00 & 0.00 & 0.00 & 0.00 & 0.00 & 0.00 & 0.00 & 0.001 & 0.001 & 0.00 & 0.00 & 0.00 \\
\hline Davidson County, Tennessee & 0.00 & 0.00 & 0.00 & 0.00 & 0.00 & 0.00 & $<0.001$ & $<0.001$ & $<0.001$ & 0.00 & 0.00 & 0.00 \\
\hline Montgomery County, Tennessee & 0.00 & 3.58 & 3.58 & 0.150 & 0.00 & 0.150 & 0.00 & 0.236 & 0.236 & 0.00 & 0.00 & 25.0 \\
\hline Robertson County, Tennessee & 5.84 & 0.00 & 5.84 & 0.00 & 0.00 & 0.00 & 0.00 & 1.370 & 1.370 & 0.00 & 0.00 & 0.360 \\
\hline Stewart County, Tennessee & 0.00 & 0.00 & 0.00 & 0.00 & 0.00 & 0.00 & 0.005 & 0.009 & 0.014 & 0.00 & 0.00 & 0.00 \\
\hline Sumner County, Tennessee & 0.00 & 0.00 & 0.00 & 0.00 & 0.00 & 0.00 & 0.00 & 0.022 & 0.022 & 0.00 & 0.00 & 0.0 \\
\hline Red total & 9.72 & 3.58 & 13.3 & 0.150 & 0.00 & 0.150 & 0.556 & 1.66 & 2.21 & 0.00 & 0.00 & 26.2 \\
\hline Barkley RCA total & 47.5 & 4.16 & 51.6 & 2.82 & 0.100 & 2.92 & 2.27 & 2.19 & 4.46 & $2,395.50$ & $\mathbf{0 . 0 0}$ & $2,453.05$ \\
\hline RCA total & 350.87 & 7.79 & 358.65 & 28.8 & 0.589 & 29.4 & 3.66 & 10.0 & 13.6 & $3,051.12$ & 0.00 & $3,370.14$ \\
\hline
\end{tabular}


Table 3. Total water use by category, reservoir catchment area, hydrologic unit, and county, Cumberland River watershed, 2010.-Continued

[Values may not add to totals because of independent rounding. All values in million gallons per day; --, no RCA associated with withdrawal; RCA, reservoir catchment area; $<$, less than; $<0.001$, actual values less than 0.001 not in table but used to sum totals. Areal extent of all reservoir catchment areas is less than the areal extent of the Cumberland River watershed]

\begin{tabular}{|c|c|c|c|c|c|c|c|c|c|c|c|c|}
\hline \multirow{3}{*}{$\begin{array}{l}\text { RCA } \\
\text { Hydrologic unit } \\
\text { County, State } \\
\text { (See figs. } 3 \text { and 4) }\end{array}$} & \multicolumn{11}{|c|}{ Water withdrawals } & \multirow{3}{*}{$\begin{array}{c}\text { Total } \\
\text { return } \\
\text { flow }\end{array}$} \\
\hline & \multicolumn{3}{|c|}{ Public supply } & \multicolumn{3}{|c|}{ Industrial } & \multicolumn{3}{|c|}{ Irrigation } & \multicolumn{2}{|c|}{ Thermoelectric } & \\
\hline & $\begin{array}{c}\text { Surface } \\
\text { water }\end{array}$ & $\begin{array}{c}\text { Ground- } \\
\text { water }\end{array}$ & Total & $\begin{array}{c}\text { Surface } \\
\text { water }\end{array}$ & $\begin{array}{l}\text { Ground- } \\
\text { water }\end{array}$ & Total & $\begin{array}{c}\text { Surface } \\
\text { water }\end{array}$ & $\begin{array}{l}\text { Ground- } \\
\text { water }\end{array}$ & Total & $\begin{array}{c}\text { Surface } \\
\text { water }\end{array}$ & $\begin{array}{l}\text { Ground- } \\
\text { water }\end{array}$ & \\
\hline \multicolumn{13}{|l|}{ Lower Cumberland 05130205} \\
\hline Livingston County, Kentucky & 1.35 & 0.00 & 1.35 & -- & -- & -- & -- & -- & -- & -- & -- & -- \\
\hline Dickson County, Kentucky & 1.37 & 0.00 & 1.37 & -- & -- & -- & -- & -- & -- & -- & -- & -- \\
\hline Logan County, Kentucky & -- & -- & -- & 1.03 & 0.00 & 1.03 & -- & -- & -- & -- & -- & -- \\
\hline Kentucky total & 69.3 & 1.47 & 70.8 & 1.66 & 0.100 & 1.76 & 2.68 & 0.058 & 2.74 & 96.4 & 0.00 & 133.72 \\
\hline Tennessee total & 282.92 & 6.32 & 289.24 & 29.2 & 0.489 & 29.7 & 0.985 & 9.89 & 10.9 & $2,954.70$ & 0.00 & $3,236.41$ \\
\hline Cumberland River watershed total & 352.21 & 7.79 & 360.00 & 30.9 & 0.589 & 31.5 & 3.66 & 9.95 & 13.6 & $3,051.12$ & 0.00 & $3,370.14$ \\
\hline
\end{tabular}




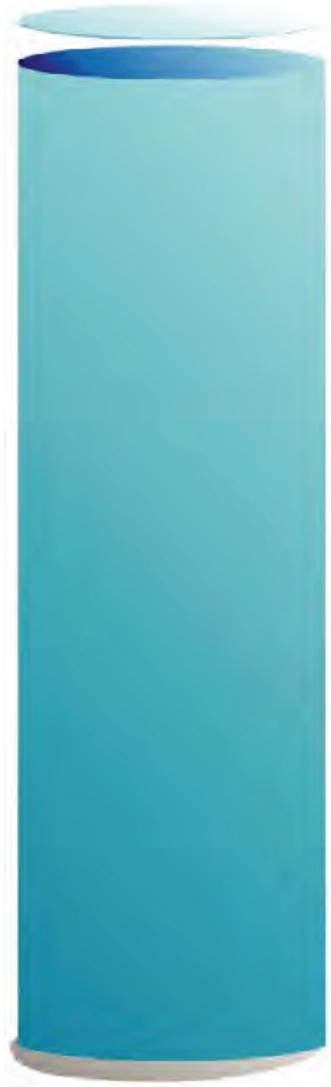

Thermoelectric

0.06

3,051.12

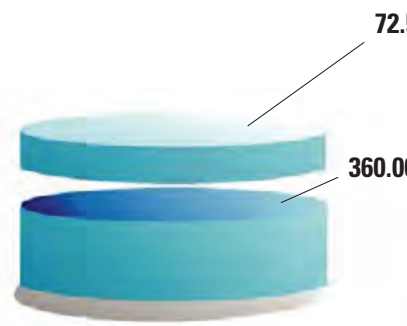

Public supply

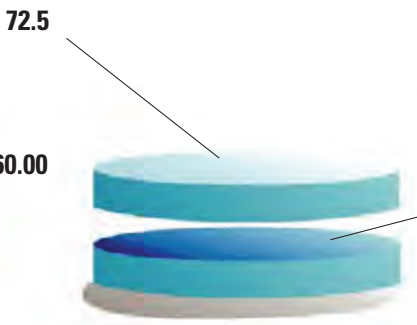

Industrial
31.5

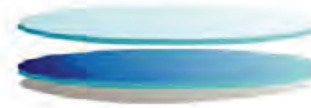

Irrigation
13.6

13.6

Note: Consumptive use is indicated in the top layer, and total water withdrawn is indicated in the lower layer. Modified from Hutson and others, 2003. Values may not add to totals because of rounding.

Figure 7. Water withdrawals and consumptive use by category, in million gallons per day, in the Cumberland River watershed in 2010.

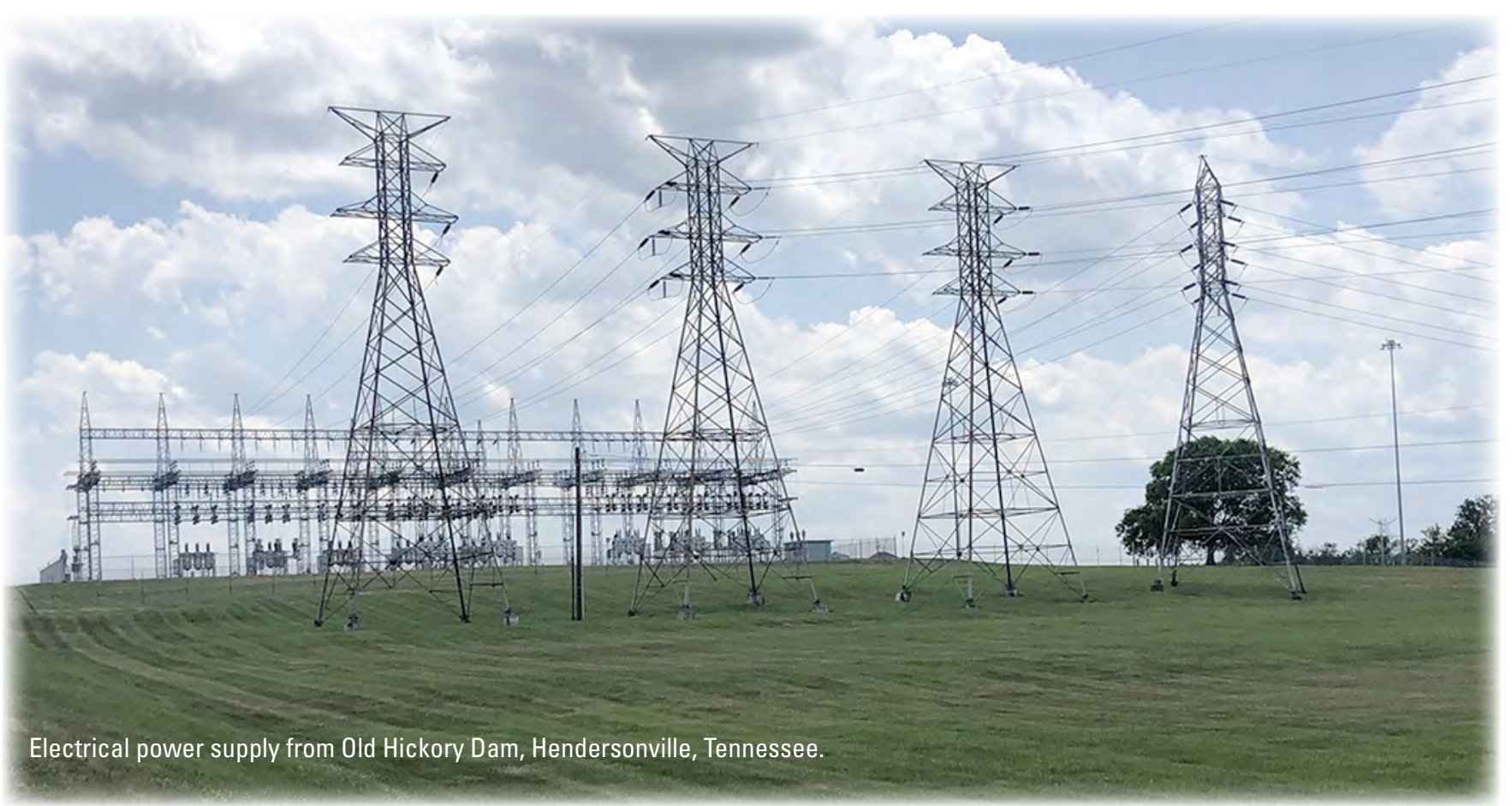




\section{Thermoelectric Power}

The relations among water availability, water use, and demographic and socioeconomic indicators over time have important implications for water use and management (Case and Alward, 1997), particularly for the thermoelectric power and industrial sectors. The electricity generated by using water from the Cumberland River watershed accounted for a large part of all electricity generated by the Tennessee Valley Authority and USACE, and the majority of the water withdrawn for thermoelectric power generation was from surface water (table 4). Water is used for condenser and reactor cooling and to replenish the boilers to produce steam.

The three thermoelectric plants located on the main stem of the Cumberland River (fig. 8) - the Cumberland Power Plant in the Barkley RCA (2,395.50 Mgal/d), the Gallatin
Fossil Plant in the Old Hickory RCA (559.20 Mgal/d), and the John Sherman Cooper Power Station in the Wolf Creek RCA (96.42 Mgal/d) - accounted for all of the water withdrawals for thermoelectric power in the Cumberland River watershed during 2010 (table 4). These three fossil-fuel plants generated 22,614.91 million kilowatt-hours in 2010 (U.S. Geological Survey, 2010) compared to 25,871.16 million kilowatt-hours in 2005 (U.S. Geological Survey, 2005). The 3-percent decrease may reflect the change in methods for calculating withdrawals and discharge between 2005 and 2010; a conversion of coal-fired plants to natural gas combined-cycle plants, which use less water (B.L. Rohrbach, U.S. Army Corps of Engineers, written commun., 2013); and (or) decreased power usage of the systems in 2010 (Diehl and Harris, 2014). Nearly all of the water used at these facilities was returned to the river.

Table 4. Thermoelectric power water use by reservoir catchment area, hydrologic unit, and county, Cumberland River watershed, 2010.

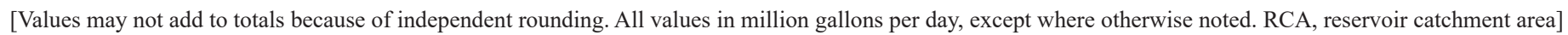

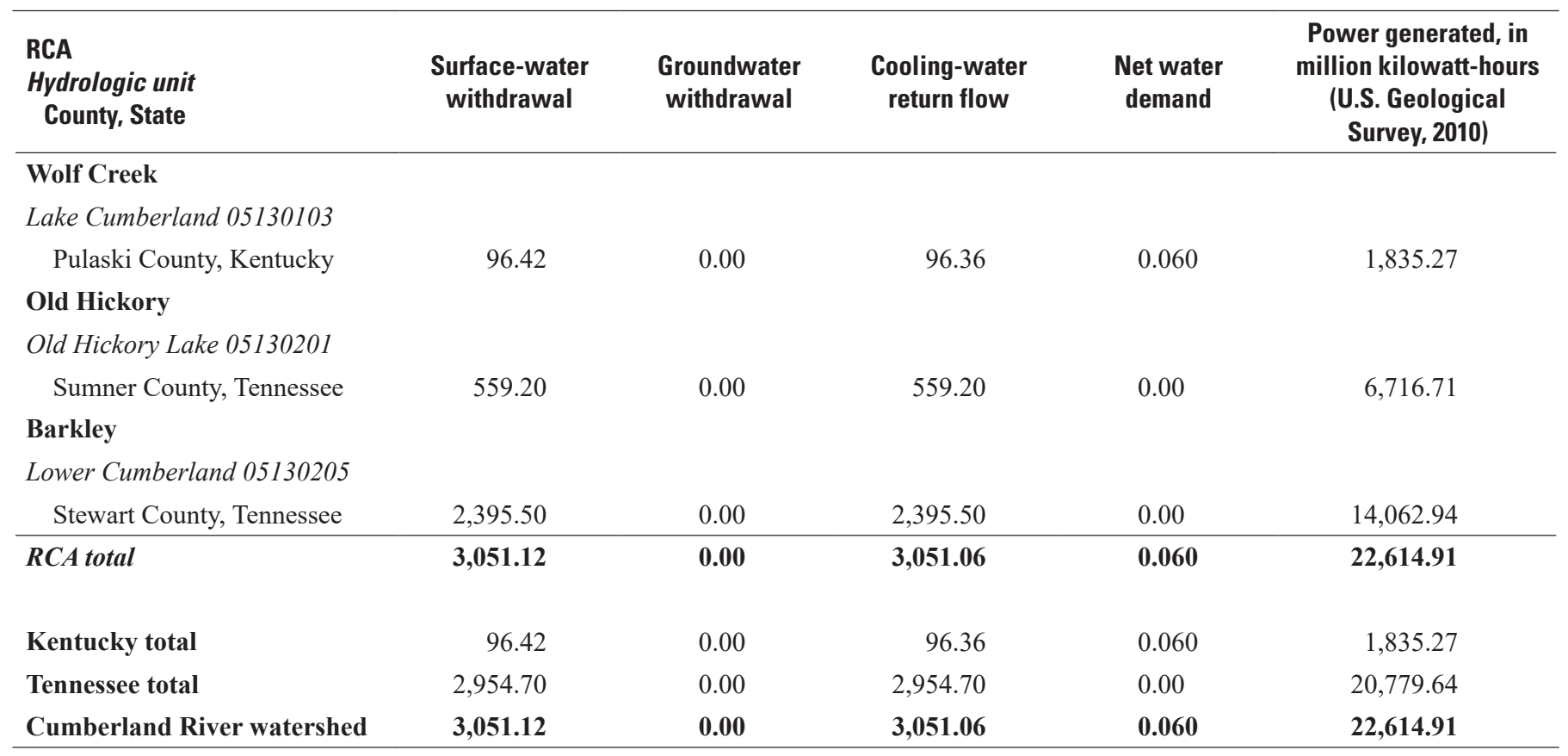

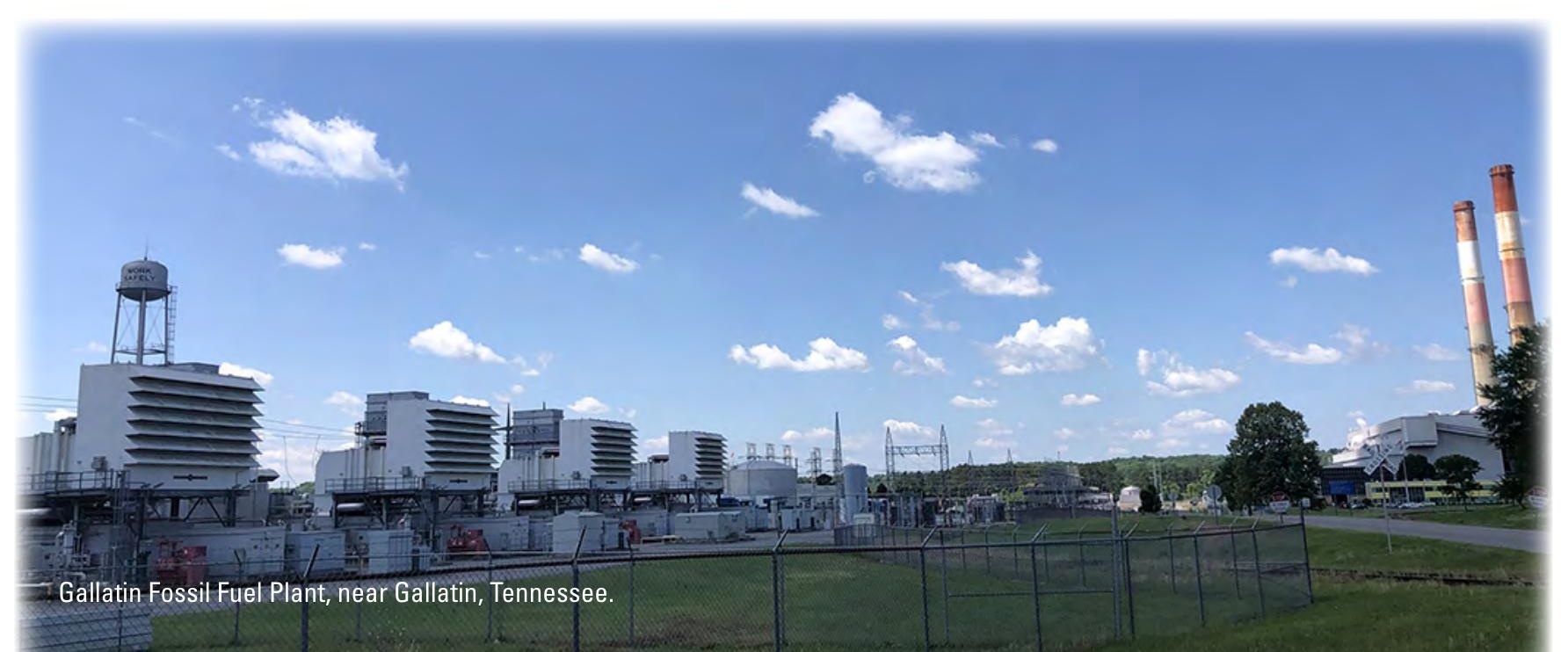




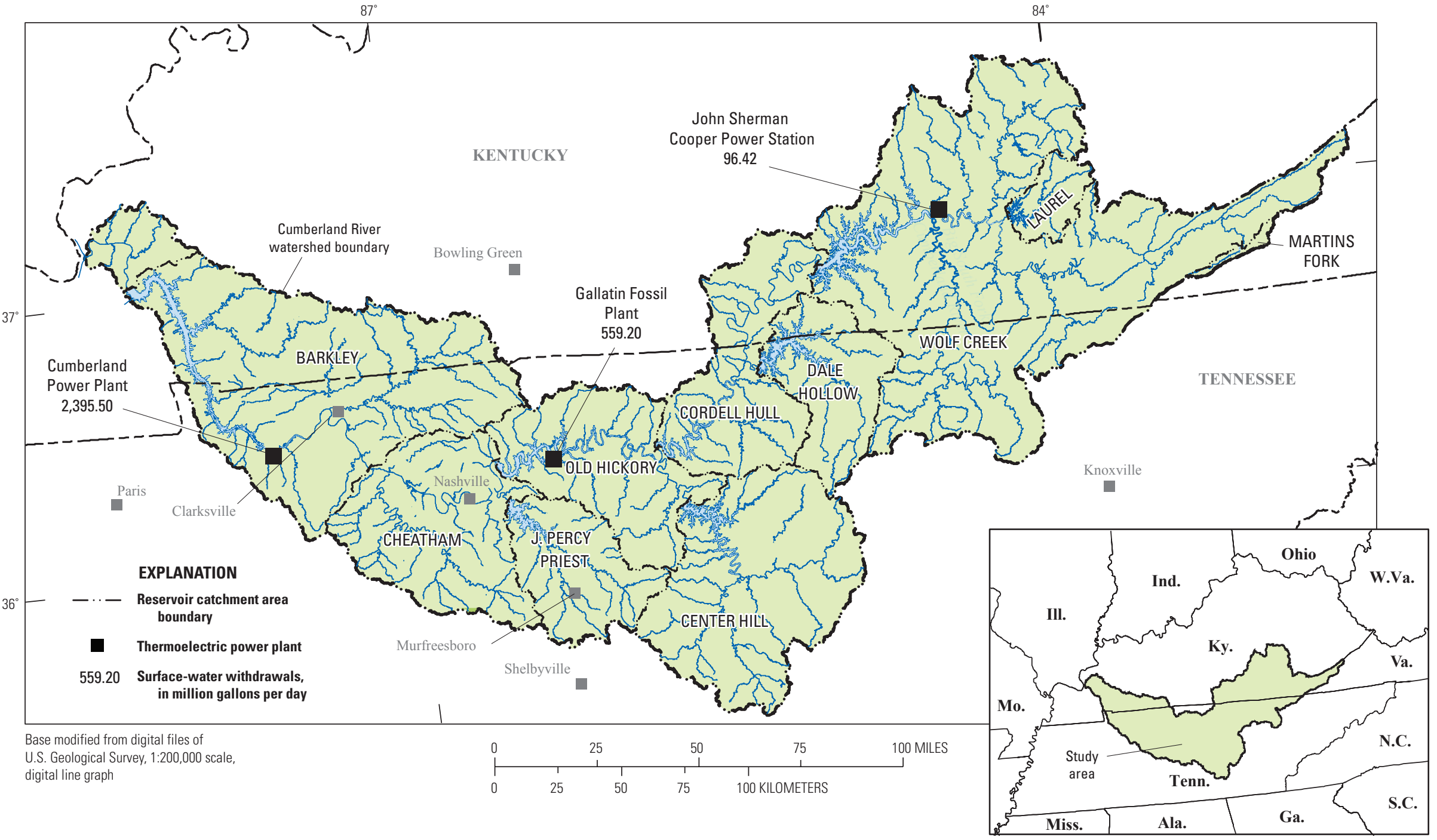

Location of the study area in Tennessee and Kentucky

Figure 8. Locations of thermoelectric power plants and surface-water withdrawals for thermoelectric use, by reservoir catchment area, in the Cumberland River watershed in 2010 . 


\section{Public Supply}

In 2010, public suppliers served water to 88 percent of the population of the Cumberland River watershed, or 2.1 million people. Water withdrawals for public supply accounted for 10 percent of the total water use and 89 percent of nonpower water use in the watershed. Surface water was the source for 98 percent, or $352.21 \mathrm{Mgal} / \mathrm{d}$, of the publicsupply total withdrawal (table 5). The remaining 2 percent, or $7.79 \mathrm{Mgal} / \mathrm{d}$, was from groundwater. The largest public-supply withdrawals, for the most part, correspond to the population centers. The Cheatham RCA provides water to Nashville, Tennessee; the Barkley RCA provides water to Clarksville, Tennessee; and the J. Percy Priest RCA provides water to Murfreesboro, Tennessee (fig. 9). Public-supply withdrawals in the aforementioned RCAs accounted for 65 percent of the total public-supply withdrawals in 2010 .
Public-supply withdrawals and wastewater releases may only indirectly relate to each other, in part because of the sewer infrastructure not being as extensive as the water distribution system, particularly in rural communities where septic tanks are more common. Other factors include the age and condition of the sanitary sewers, the presence of combined sewers, and the amount of infiltration and inflow of groundwater or stormwater into the sewer system (B.L. Rohrbach, U.S. Army Corps of Engineers, written commun., 2013). The balance between public-supply withdrawals and wastewater releases may be affected by how industrial water is disposed. For example, water that is released from a self-supplied industrial facility may be conveyed to a publicly owned treatment works instead of discharged directly to a stream. For this report, estimates of public-supply wastewater releases and estimates of industrial wastewater are included as part of the total return flows.

Table 5. Public-supply water withdrawals by reservoir catchment area, hydrologic unit, and county, Cumberland River watershed, 2010.

[Values may not add to totals because of independent rounding. All values in million gallons per day. Areal extent of all reservoir catchment areas is less than the areal extent of the Cumberland River watershed. RCA, reservoir catchment area]

\begin{tabular}{lccc}
\hline $\begin{array}{l}\text { RCA } \\
\text { Hydrologic unit } \\
\text { County, State }\end{array}$ & $\begin{array}{c}\text { Surface-water } \\
\text { withdrawal }\end{array}$ & $\begin{array}{c}\text { Groundwater } \\
\text { withdrawal }\end{array}$ & $\begin{array}{c}\text { Total } \\
\text { water }\end{array}$ \\
\hline $\begin{array}{l}\text { Martins Fork } \\
\text { Upper Cumberland 05130101 }\end{array}$ & & & \\
$\quad$ Harlan County, Kentucky & 2.30 & 0.00 & 2.30 \\
\hline Upper Cumberland total & 2.30 & 0.00 & 2.30 \\
Martins Fork RCA total & $\mathbf{2 . 3 0}$ & $\mathbf{0 . 0 0}$ & $\mathbf{2 . 3 0}$
\end{tabular}

\section{Laurel}

Upper Cumberland 05130101

Laurel County, Kentucky

Whitley County, Kentucky

Upper Cumberland total

Laurel RCA total

2.30

$\mathbf{0 . 0 0}$

2.30

\section{Wolf Creek}

Upper Cumberland 05130101

Bell County, Kentucky

Harlan County, Kentucky

Knox County, Kentucky

McCreary County, Kentucky

Whitley County, Kentucky

Campbell County, Tennessee

Claiborne County, Tennessee

Upper Cumberland total

\begin{tabular}{lll}
5.01 & 0.00 & 5.01 \\
3.82 & 0.00 & 3.82 \\
\hline 8.83 & 0.00 & 8.83 \\
$\mathbf{8 . 8 3}$ & $\mathbf{0 . 0 0}$ & $\mathbf{8 . 8 3}$ \\
& & \\
& & \\
& & \\
3.84 & & 3.84 \\
0.00 & 0.00 & 1.36 \\
0.326 & 1.36 & 0.326 \\
1.17 & 0.00 & 1.17 \\
1.55 & 0.00 & 1.55 \\
0.954 & 0.00 & 0.954 \\
0.00 & 0.00 & 0.151 \\
\hline 7.83 & 0.151 & 9.34
\end{tabular}


Table 5. Public-supply water withdrawals by reservoir catchment area, hydrologic unit, and county, Cumberland River watershed, 2010.-Continued

[Values may not add to totals because of independent rounding. All values in million gallons per day. Areal extent of all reservoir catchment areas is less than the areal extent of the Cumberland River watershed. RCA, reservoir catchment area]

\begin{tabular}{|c|c|c|c|}
\hline $\begin{array}{l}\text { RCA } \\
\text { Hydrologic unit } \\
\text { County, State }\end{array}$ & $\begin{array}{l}\text { Surface-water } \\
\text { withdrawal }\end{array}$ & $\begin{array}{l}\text { Groundwater } \\
\text { withdrawal }\end{array}$ & $\begin{array}{l}\text { Total } \\
\text { water }\end{array}$ \\
\hline \multicolumn{4}{|l|}{ Rockcastle 05130102} \\
\hline Jackson County, Kentucky & 1.80 & 0.00 & 1.80 \\
\hline Laurel County, Kentucky & 4.09 & 0.00 & 4.09 \\
\hline Rockcastle County, Kentucky & 2.34 & 0.00 & 2.34 \\
\hline Rockcastle total & 8.23 & 0.00 & 8.23 \\
\hline \multicolumn{4}{|l|}{ Lake Cumberland 05130103} \\
\hline Clinton County, Kentucky & 3.40 & 0.00 & 3.40 \\
\hline Lincoln County, Kentucky & 0.277 & 0.00 & 0.277 \\
\hline Pulaski County, Kentucky & 9.04 & 0.00 & 9.04 \\
\hline Russell County, Kentucky & 3.48 & 0.00 & 3.48 \\
\hline Wayne County, Kentucky & 2.46 & 0.00 & 2.46 \\
\hline Lake Cumberland total & 18.7 & 0.00 & 18.7 \\
\hline \multicolumn{4}{|l|}{ South Fork Cumberland 05130104} \\
\hline McCreary County, Kentucky & 0.722 & 0.00 & 0.722 \\
\hline Pulaski County, Kentucky & 0.054 & 0.00 & 0.054 \\
\hline Fentress County, Tennessee & 1.74 & 0.00 & 1.74 \\
\hline Scott County, Tennessee & 2.58 & 0.00 & 2.58 \\
\hline South Fork Cumberland total & 5.09 & 0.00 & 5.09 \\
\hline Wolf Creek RCA total & 39.8 & 1.51 & 41.3 \\
\hline
\end{tabular}

\section{Dale Hollow}

Obey 05130105

\begin{tabular}{llll}
\multicolumn{1}{c}{ Pickett County, Tennessee } & 0.620 & 0.00 & 0.620 \\
Putnam County, Tennessee & 1.27 & 0.008 & 1.28 \\
\hline Obey total & 1.89 & 0.008 & 1.90 \\
Dale Hollow RCA total & $\mathbf{1 . 8 9}$ & $\mathbf{0 . 0 0 8}$ & $\mathbf{1 . 9 0}$
\end{tabular}

\section{Cordell Hull}

Lake Cumberland 05130103

\begin{tabular}{cccc} 
Cumberland County, Kentucky & 0.776 & 0.00 & 0.776 \\
\hline Lake Cumberland total & 0.776 & 0.00 & 0.776
\end{tabular}

\begin{tabular}{llll} 
Cordell Hull 05130106 & & & \\
Clay County, Tennessee & 0.466 & 0.00 & 0.466 \\
Jackson County, Tennessee & 0.539 & 0.00 & 0.539 \\
Overton County, Tennessee & 2.24 & 0.00 & 2.24 \\
\hline Cordell Hull total & 3.25 & 0.00 & 3.25
\end{tabular}


Table 5. Public-supply water withdrawals by reservoir catchment area, hydrologic unit, and county, Cumberland River watershed, 2010.-Continued

[Values may not add to totals because of independent rounding. All values in million gallons per day. Areal extent of all reservoir catchment areas is less than the areal extent of the Cumberland River watershed. RCA, reservoir catchment area]

\begin{tabular}{|c|c|c|c|}
\hline $\begin{array}{l}\text { RCA } \\
\text { Hydrologic unit } \\
\text { County, State }\end{array}$ & $\begin{array}{c}\text { Surface-water } \\
\text { withdrawal }\end{array}$ & $\begin{array}{l}\text { Groundwater } \\
\text { withdrawal }\end{array}$ & $\begin{array}{l}\text { Total } \\
\text { water }\end{array}$ \\
\hline \multicolumn{4}{|l|}{ Obey 05130105} \\
\hline Clay County, Tennessee & 0.694 & 0.00 & 0.694 \\
\hline Obey total & 0.694 & 0.00 & 0.694 \\
\hline Cordell Hull RCA total & 4.72 & 0.00 & 4.72 \\
\hline \multicolumn{4}{|l|}{ Center Hill } \\
\hline \multicolumn{4}{|l|}{ Collins 05130107} \\
\hline Grundy County, Tennessee & 0.862 & 0.00 & 0.862 \\
\hline Warren County, Tennessee & 5.10 & 0.00 & 5.10 \\
\hline Collins total & 5.96 & 0.00 & 5.96 \\
\hline \multicolumn{4}{|l|}{ Caney 05130108} \\
\hline Bledsoe County, Tennessee & 0.729 & 0.00 & 0.729 \\
\hline Putnam County, Tennessee & 13.3 & 0.00 & 13.3 \\
\hline Van Buren County, Tennessee & 0.59 & 0.00 & 0.59 \\
\hline White County, Tennessee & 3.54 & 0.00 & 3.54 \\
\hline Caney total & 18.1 & 0.00 & 18.1 \\
\hline Center Hill RCA total & 24.1 & 0.00 & 24.1 \\
\hline
\end{tabular}

\section{Old Hickory}

Caney 05130108

\begin{tabular}{cccc} 
DeKalb County, Tennessee & 1.81 & 0.073 & 1.89 \\
\hline Caney total & 1.81 & 0.073 & 1.89
\end{tabular}

Old Hickory Lake 05130201

$\begin{array}{llll}\text { Davidson County, Tennessee } & 0.834 & 0.00 & 0.834\end{array}$

$\begin{array}{llll}\text { Smith County, Tennessee } & 2.09 & 0.00 & 2.09\end{array}$

$\begin{array}{llll}\text { Sumner County, Tennessee } & 23.8 & 0.00 & 23.8\end{array}$

$\begin{array}{llll}\text { Trousdale County, Tennessee } & 0.922 & 0.00 & 0.922\end{array}$

\begin{tabular}{cccc} 
Wilson County, Tennessee & 13.5 & 0.211 & 13.7 \\
\hline Old Hickory Lake total & 41.2 & 0.211 & 41.4
\end{tabular}

Old Hickory RCA total

43.0

0.283

43.3

\section{J. Percy Priest}

Stones 05130203

\begin{tabular}{lccc} 
Cannon County, Tennessee & 1.14 & 0.00 & 1.14 \\
Rutherford County, Tennessee & 33.8 & 0.00 & 33.8 \\
Wilson County, Tennessee & 0.00 & 1.71 & 1.71 \\
\hline Stones total & 34.9 & 1.71 & 36.6 \\
J. Percy Priest RCA total & $\mathbf{3 4 . 9}$ & $\mathbf{1 . 7 1}$ & $\mathbf{3 6 . 6}$
\end{tabular}


Table 5. Public-supply water withdrawals by reservoir catchment area, hydrologic unit, and county, Cumberland River watershed, 2010.-Continued

[Values may not add to totals because of independent rounding. All values in million gallons per day. Areal extent of all reservoir catchment areas is less than the areal extent of the Cumberland River watershed. RCA, reservoir catchment area]

\begin{tabular}{|c|c|c|c|}
\hline $\begin{array}{l}\text { RCA } \\
\text { Hydrologic unit } \\
\text { County, State }\end{array}$ & $\begin{array}{l}\text { Surface-water } \\
\text { withdrawal }\end{array}$ & $\begin{array}{l}\text { Groundwater } \\
\text { withdrawal }\end{array}$ & $\begin{array}{l}\text { Total } \\
\text { water }\end{array}$ \\
\hline \multicolumn{4}{|l|}{ Cheatham } \\
\hline \multicolumn{4}{|l|}{ Sycamore 05130202} \\
\hline Cheatham County, Tennessee & 2.30 & 0.00 & 2.30 \\
\hline Davidson County, Tennessee & 135.54 & 0.00 & 135.54 \\
\hline Sycamore total & 137.85 & 0.00 & 137.85 \\
\hline \multicolumn{4}{|l|}{ Harpeth 05130204} \\
\hline Cheatham County, Tennessee & 0.652 & 0.00 & 0.652 \\
\hline Dickson County, Tennessee & 3.67 & 0.00 & 3.67 \\
\hline Williamson County, Tennessee & 1.69 & 0.112 & 1.80 \\
\hline Harpeth total & 6.01 & 0.112 & 6.12 \\
\hline Cheatham RCA total & 143.85 & 0.112 & 143.97 \\
\hline \multicolumn{4}{|l|}{ Barkley } \\
\hline \multicolumn{4}{|l|}{ Lower Cumberland 05130205} \\
\hline Lyon County, Kentucky & 2.68 & 0.113 & 2.79 \\
\hline Trigg County, Kentucky & 14.9 & 0.000 & 14.9 \\
\hline Dickson County, Tennessee & 0.019 & 0.019 & 0.039 \\
\hline Houston County, Tennessee & 1.05 & 0.00 & 1.05 \\
\hline Montgomery County, Tennessee & 18.8 & 0.00 & 18.8 \\
\hline Stewart County, Tennessee & 0.273 & 0.455 & 0.728 \\
\hline Lower Cumberland total & 37.7 & 0.587 & 38.3 \\
\hline \multicolumn{4}{|l|}{ Red 05130206} \\
\hline Todd County, Tennessee & 3.88 & 0.00 & 3.88 \\
\hline Montgomery County, Tennessee & 0.00 & 3.58 & 3.58 \\
\hline Robertson County, Tennessee & 5.84 & 0.00 & 5.84 \\
\hline Red total & 9.72 & 3.58 & 13.3 \\
\hline Barkley RCA total & 47.5 & 4.16 & 51.6 \\
\hline RCA total & 350.87 & 7.79 & 358.65 \\
\hline \multicolumn{4}{|l|}{ Lower Cumberland 05130205} \\
\hline Livingston County, Kentucky ${ }^{1}$ & 1.35 & 0.00 & 1.35 \\
\hline Kentucky total (18.6 percent) & 69.3 & 1.47 & 70.8 \\
\hline Tennessee total ( 81.4 percent) & 282.92 & 6.32 & 289.24 \\
\hline Cumberland River watershed total & 352.21 & 7.79 & 360.00 \\
\hline
\end{tabular}

${ }^{1}$ No reservoir catchment associated with withdrawal. 


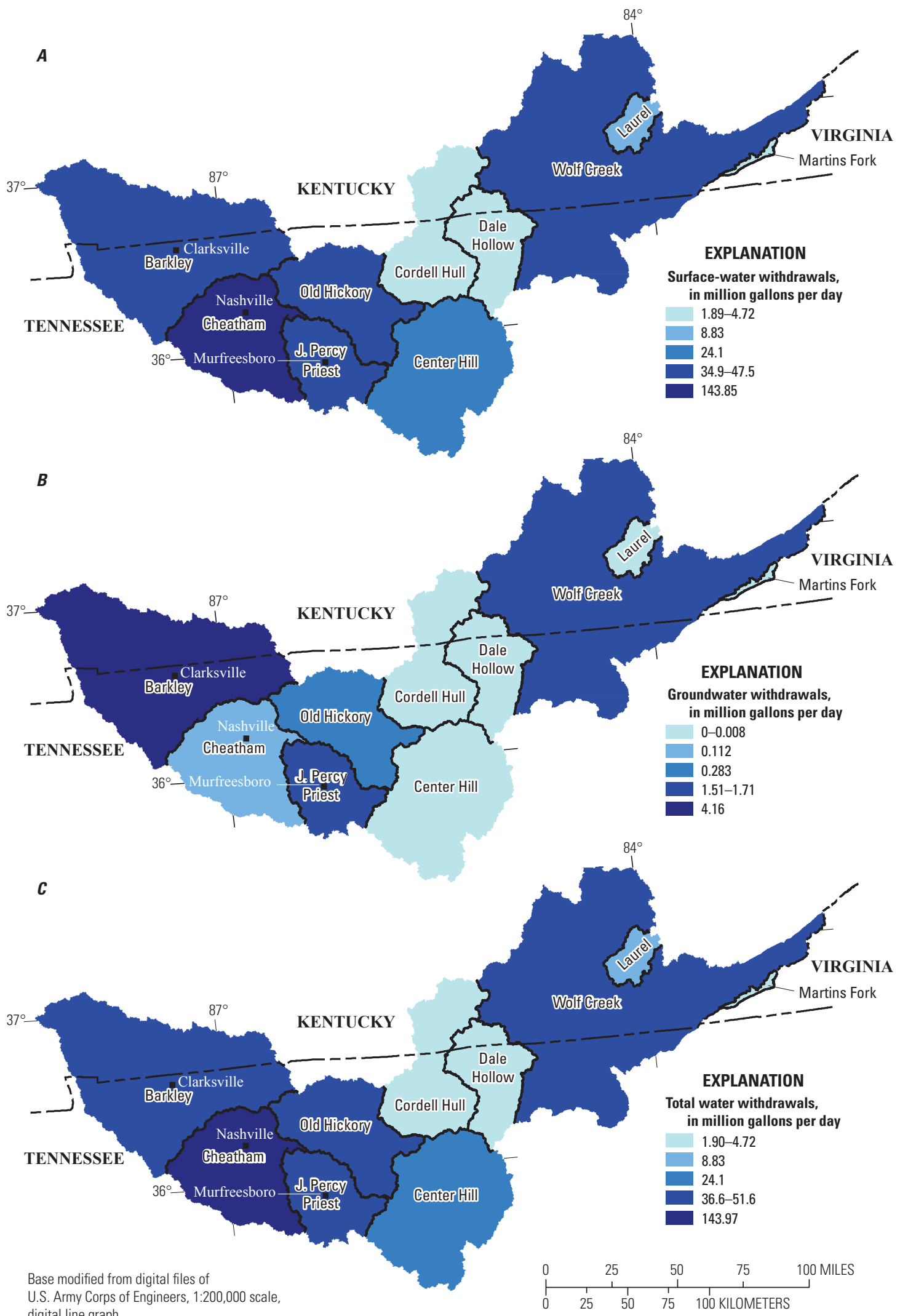
digital line graph

Figure 9. Public-supply water withdrawals by source, $(A)$ surface water, $(B)$ groundwater, and $(C)$ total water, and by reservoir catchment area in the Cumberland River watershed in 2010. 


\section{Industrial}

Industrial water withdrawals in the Cumberland River watershed, which were mostly from surface water, generally were greatest in the western and northern parts of the watershed (fig. 10). Water withdrawals for industrial use during 2010 were estimated to be $31.5 \mathrm{Mgal} / \mathrm{d}$ (tables 1 and 6), which is 1 percent of the total water withdrawals and 8 percent of the nonpower water withdrawals. Surface water supplied more than 98 percent of the water, $30.9 \mathrm{Mgal} / \mathrm{d}$, for industrial purposes, and groundwater supplied less than 2 percent, $0.589 \mathrm{Mgal} / \mathrm{d}$. Industrial water withdrawals in the Old Hickory RCA were $18.3 \mathrm{Mgal} / \mathrm{d}$ and accounted for 58 percent of the industrial water withdrawals (table 6).

Industrial water use includes water used for such purposes as processing, washing, and cooling in facilities that manufacture various products. In 2010 in the Cumberland River watershed, the major water-using industry was the manufacturing of chemical and allied products, which accounted for 54 percent (16.9 Mgal/d) of the industrial water withdrawals. Industrial return flow is water disposed from use in sanitary, process, or cooling activities and excludes stormwater runoff. Return-flow data for industry were obtained from the EPA, National Pollutant Discharge Elimination System, Permit Compliance System. A strict sitespecific accounting of industrial withdrawals and return flows is difficult because of the different ways water is obtained and disposed. For example, industries that purchase water from a public supplier may discharge to a stream and bypass the wastewater-treatment plant, or self-supplied industries may release water to a wastewater-treatment plant rather than to a stream. Uncertainty about the amount of return flow also may result if an industry includes estimates of stormwater runoff in the sanitary, process, or cooling-water return-flow volumes. Meter registration errors also may occur.

\section{Irrigation}

The estimated quantity of water withdrawn for irrigation during 2010 was $13.6 \mathrm{Mgal} / \mathrm{d}$ (table 7) and represented less than 1 percent of the total water withdrawals and 3 percent of the nonpower water withdrawals in the Cumberland River watershed. Surface water was the source of water for 27 percent of the irrigation water withdrawals; groundwater was the source of the remaining 73 percent. The most intensive irrigation in the watershed was in the Center Hill RCA, which accounted for 41 percent of the total, or $5.64 \mathrm{Mgal} / \mathrm{d}$, in 2010 (table 7; fig. 11). More than 80 percent of the total irrigation in the Cumberland River watershed occurred in Tennessee in 2010. Irrigation water was primarily applied by sprinkler and micro-irrigation systems. All water withdrawn for irrigation is assumed to be fully consumed with no return flow.

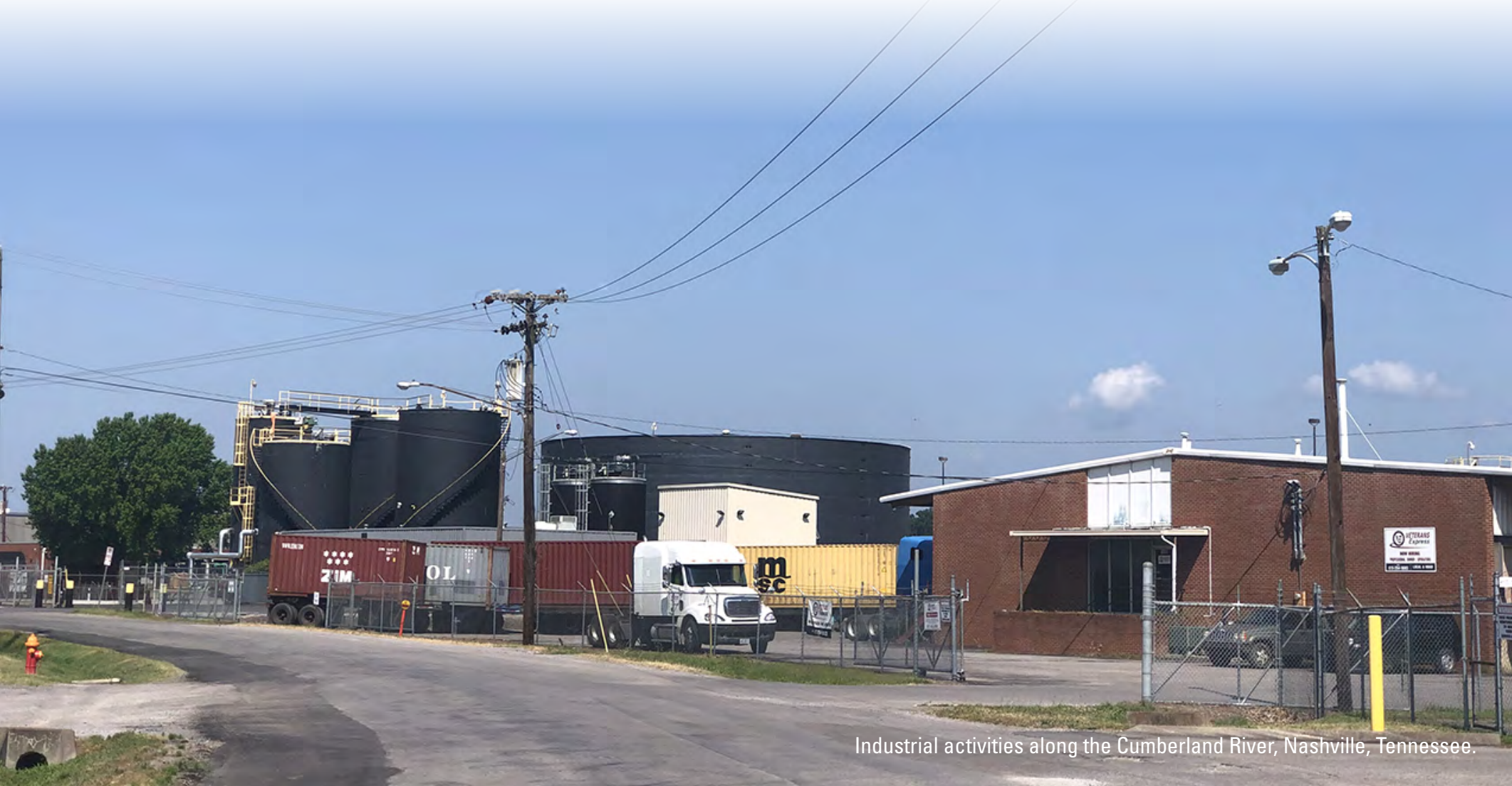


Table 6. Industrial water withdrawals by reservoir catchment area, hydrologic unit, and county, Cumberland River watershed, 2010.

[Values may not add to totals because of independent rounding. All values in million gallons per day. Areal extent of all reservoir catchment areas is less than the areal extent of the Cumberland River watershed. RCA, reservoir catchment area]

\begin{tabular}{|c|c|c|c|}
\hline $\begin{array}{l}\text { RCA } \\
\text { Hydrologic unit } \\
\text { County, State }\end{array}$ & $\begin{array}{c}\text { Surface-water } \\
\text { withdrawal }\end{array}$ & $\begin{array}{l}\text { Groundwater } \\
\text { withdrawal }\end{array}$ & $\begin{array}{c}\text { Total } \\
\text { water }\end{array}$ \\
\hline \multicolumn{4}{|l|}{ Wolf Creek } \\
\hline \multicolumn{4}{|l|}{ South Fork Cumberland 05130104} \\
\hline Pulaski County, Kentucky & 0.240 & 0.00 & 0.240 \\
\hline South Fork Cumberland total & 0.240 & 0.00 & 0.240 \\
\hline Wolf Creek RCA total & 0.240 & 0.00 & 0.240 \\
\hline \multicolumn{4}{|l|}{ Cordell Hull } \\
\hline \multicolumn{4}{|l|}{ Cordell Hull 05130106} \\
\hline Jackson County, Tennessee & 0.00 & 0.037 & 0.037 \\
\hline Putnam County, Tennessee & 0.210 & 0.00 & 0.210 \\
\hline Cordell Hull total & 0.210 & 0.037 & 0.247 \\
\hline Cordell Hull RCA total & 0.210 & $\mathbf{0 . 0 3 7}$ & 0.247 \\
\hline \multicolumn{4}{|l|}{ Old Hickory } \\
\hline \multicolumn{4}{|l|}{ Old Hickory Lake 05130201} \\
\hline Davidson County, Tennessee & 16.9 & 0.00 & 16.9 \\
\hline Sumner County, Tennessee & 1.01 & 0.00 & 1.01 \\
\hline Wilson County, Tennessee & 0.402 & 0.00 & 0.402 \\
\hline Old Hickory Lake total & 18.3 & 0.00 & 18.3 \\
\hline Old Hickory RCA total & 18.3 & 0.00 & 18.3 \\
\hline
\end{tabular}

\section{J. Percy Priest}

Stones 05130203

\begin{tabular}{llll}
\multicolumn{1}{c}{ Davidson County, Tennessee } & 0.000 & 0.000 & 0.00 \\
Rutherford County, Tennessee & 0.469 & 0.016 & 0.485 \\
\hline Stones total & 0.469 & 0.016 & 0.485 \\
J. Percy Priest RCA total & $\mathbf{0 . 4 6 9}$ & $\mathbf{0 . 0 1 6}$ & $\mathbf{0 . 4 8 5}$
\end{tabular}

\section{Cheatham}

Sycamore 05130202

\begin{tabular}{clll} 
Cheatham County, Tennessee & 0.188 & 0.00 & 0.188 \\
Davidson County, Tennessee & 8.32 & 0.436 & 8.76 \\
\hline Sycamore total & 8.51 & 0.436 & 8.94
\end{tabular}

Stones 05130203

\begin{tabular}{llll} 
Davidson County, Tennessee & 0.008 & 0.00 & 0.008 \\
\hline Stones total & 0.008 & 0.00 & 0.008
\end{tabular}


Table 6. Industrial water withdrawals by reservoir catchment area, hydrologic unit, and county, Cumberland River watershed, 2010._-Continued

[Values may not add to totals because of independent rounding. All values in million gallons per day. Areal extent of all reservoir catchment areas is less than the areal extent of the Cumberland River watershed. RCA, reservoir catchment area]

\begin{tabular}{|c|c|c|c|}
\hline $\begin{array}{l}\text { RCA } \\
\text { Hydrologic unit } \\
\text { County, State }\end{array}$ & $\begin{array}{c}\text { Surface-water } \\
\text { withdrawal }\end{array}$ & $\begin{array}{l}\text { Groundwater } \\
\text { withdrawal }\end{array}$ & $\begin{array}{l}\text { Total } \\
\text { water }\end{array}$ \\
\hline \multicolumn{4}{|l|}{ Harpeth 05130204} \\
\hline Cheatham County, Tennessee & 0.339 & 0.00 & 0.339 \\
\hline Cheatham RCA total & 8.85 & 0.436 & 9.29 \\
\hline \multicolumn{4}{|l|}{ Barkley } \\
\hline \multicolumn{4}{|l|}{ Lower Cumberland 05130205} \\
\hline Christian County, Kentucky & 1.41 & 0.00 & 1.41 \\
\hline Lyon County, Kentucky & 0.010 & 0.00 & 0.010 \\
\hline Todd County, Kentucky & 0.00 & 0.100 & 0.100 \\
\hline Montgomery County, Kentucky & 1.25 & 0.00 & 1.25 \\
\hline Lower Cumberland total & 2.67 & 0.100 & 2.77 \\
\hline \multicolumn{4}{|l|}{ Red 05130206} \\
\hline Montgomery County, Kentucky & 0.150 & 0.00 & 0.150 \\
\hline Robertson County, Kentucky & 0.00 & 0.00 & 0.00 \\
\hline Red total & 0.150 & 0.00 & 0.150 \\
\hline Barkley RCA total & 2.82 & 0.100 & 2.92 \\
\hline RCA total & 30.9 & 0.589 & 31.5 \\
\hline Logan County, Kentucky ${ }^{1}$ & 1.03 & 0.00 & 1.03 \\
\hline Kentucky total & 1.66 & 0.100 & 1.76 \\
\hline Tennessee total & 29.2 & 0.489 & 29.7 \\
\hline Cumberland River watershed total & 30.9 & 0.589 & 31.5 \\
\hline
\end{tabular}

${ }^{1}$ Withdrawal located outside of the Cumberland River watershed. 

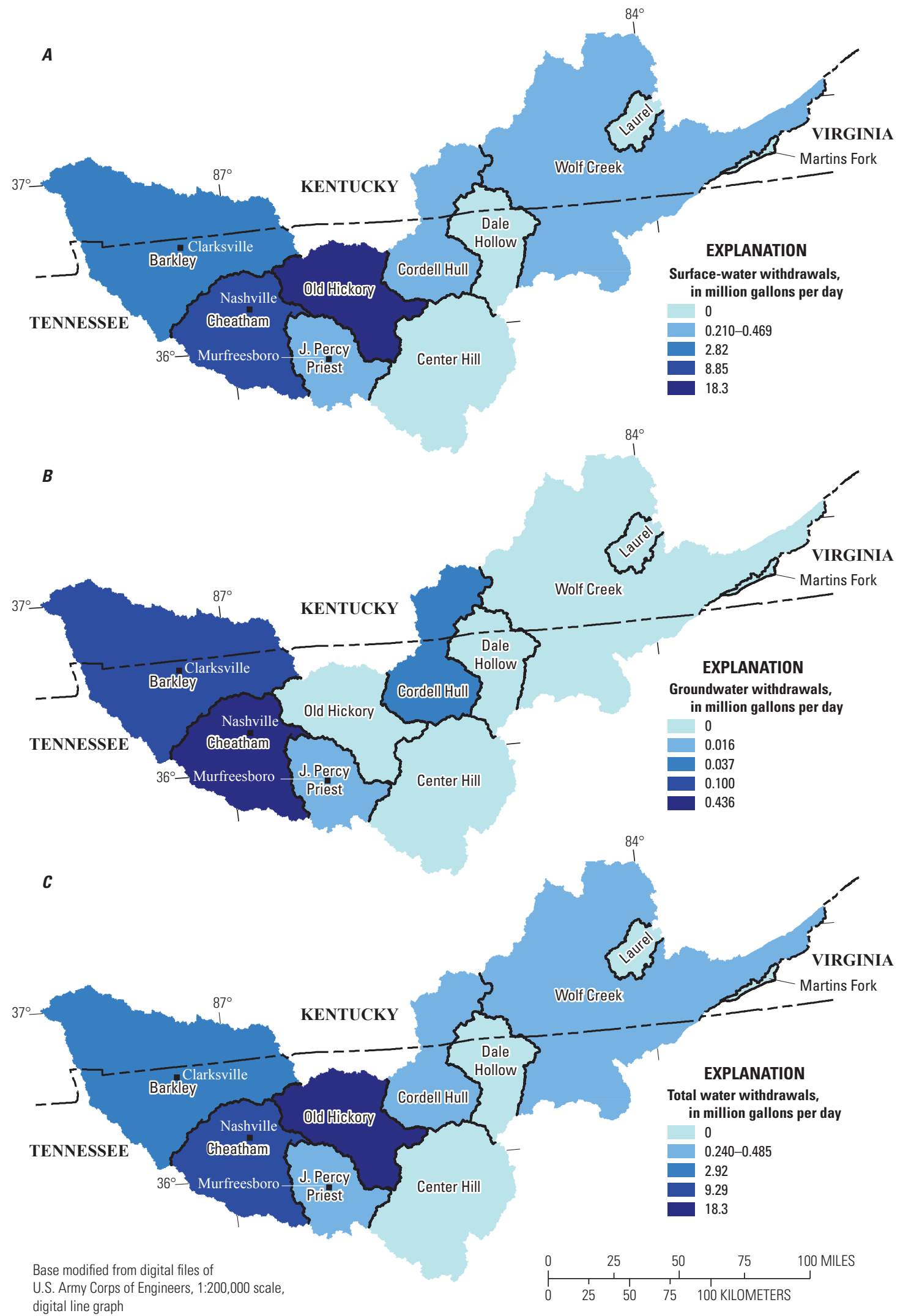

Figure 10. Industrial water withdrawals by source, $(A)$ surface water, $(B)$ groundwater, and $(C)$ total water, and by reservoir catchment area in the Cumberland River watershed in 2010. 
Table 7. Irrigation water withdrawals by reservoir catchment area, hydrologic unit, and county, Cumberland River watershed, 2010.

[Values may not add to totals because of independent rounding. All values in million gallons per day; $<$, less than; $<0.001$, actual values less than 0.001 not in table but used to sum totals. Areal extent of all reservoir catchment areas is less than the areal extent of the Cumberland River watershed. RCA, reservoir catchment area]

\begin{tabular}{lccc}
\hline $\begin{array}{l}\text { RCA } \\
\text { Hydrologic unit } \\
\text { County, State }\end{array}$ & $\begin{array}{c}\text { Surface-water } \\
\text { withdrawal }\end{array}$ & $\begin{array}{c}\text { Groundwater } \\
\text { withdrawal }\end{array}$ & $\begin{array}{c}\text { Total } \\
\text { water }\end{array}$ \\
\hline $\begin{array}{l}\text { Martins Fork } \\
\text { Upper Cumberland 05130101 }\end{array}$ & & & \\
Bell County, Kentucky & $<0.001$ & 0.00 & $<0.001$ \\
Hardin County, Kentucky & ${ }^{1} 0.00$ & ${ }^{1} 0.00$ & 0.00 \\
\hline Upper Cumberland total & $<0.001$ & 0.00 & $<0.001$ \\
Martins Fork RCA total & $<\mathbf{0 . 0 0 1}$ & $\mathbf{0 . 0 0}$ & $<\mathbf{0 . 0 0 1}$
\end{tabular}

\section{Laurel}

Upper Cumberland 05130101

\begin{tabular}{lccc} 
Laurel County, Kentucky & 0.009 & 0.00 & 0.009 \\
Whitley County, Kentucky & 10.00 & ${ }^{1} 0.00$ & 0.00 \\
\hline Upper Cumberland total & 0.009 & 0.00 & 0.009 \\
Laurel RCA total & $\mathbf{0 . 0 0 9}$ & $\mathbf{0 . 0 0}$ & $\mathbf{0 . 0 0 9}$
\end{tabular}

\section{Wolf Creek}

\begin{tabular}{|c|c|c|c|}
\hline \multicolumn{4}{|l|}{ Upper Cumberland 05130101} \\
\hline Bell County, Kentucky & 0.029 & 0.00 & 0.029 \\
\hline Clay County, Kentucky & $<0.001$ & 0.00 & $<0.001$ \\
\hline Hardin County, Kentucky & ${ }^{1} 0.00$ & ${ }^{1} 0.00$ & 0.00 \\
\hline Laurel County, Kentucky & $<0.001$ & 0.00 & $<0.001$ \\
\hline Leslie County, Kentucky & ${ }^{1} 0.00$ & ${ }^{1} 0.00$ & 0.00 \\
\hline Letcher County, Kentucky & ${ }^{1} 0.00$ & ${ }^{1} 0.00$ & 0.00 \\
\hline McCreary County, Kentucky & 0.017 & 0.00 & 0.017 \\
\hline Whitley County, Kentucky & 0.00 & 0.00 & 0.00 \\
\hline Campbell County, Tennessee & 0.00 & 0.021 & 0.021 \\
\hline Claiborne County, Tennessee & 0.00 & 0.005 & 0.005 \\
\hline Scott County, Tennessee & ${ }^{1} 0.00$ & ${ }^{1} 0.00$ & 0.00 \\
\hline Upper Cumberland total & 0.046 & 0.026 & 0.072 \\
\hline \multicolumn{4}{|l|}{ Rockcastle 05130102} \\
\hline Clay County, Kentucky & $<0.001$ & 0.00 & $<0.001$ \\
\hline Jackson County, Kentucky & 0.006 & 0.00 & 0.006 \\
\hline Laurel County, Kentucky & 0.011 & 0.00 & 0.011 \\
\hline Madison County, Kentucky & $<0.001$ & $<0.001$ & $<0.001$ \\
\hline Pulaski County, Kentucky & 0.019 & 0.00 & 0.019 \\
\hline Rockcastle County, Kentucky & 0.008 & 0.00 & 0.008 \\
\hline Rockcastle total & 0.044 & 0.000 & 0.044 \\
\hline
\end{tabular}


Table 7. Irrigation water withdrawals by reservoir catchment area, hydrologic unit, and county, Cumberland River watershed, 2010. - Continued

[Values may not add to totals because of independent rounding. All values in million gallons per day; $<$, less than; $<0.001$, actual values less than 0.001 not in table but used to sum totals. Areal extent of all reservoir catchment areas is less than the areal extent of the Cumberland River watershed. RCA, reservoir catchment area]

\begin{tabular}{|c|c|c|c|}
\hline $\begin{array}{l}\text { RCA } \\
\text { Hydrologic unit } \\
\text { County, State }\end{array}$ & $\begin{array}{c}\text { Surface-water } \\
\text { withdrawal }\end{array}$ & $\begin{array}{l}\text { Groundwater } \\
\text { withdrawal }\end{array}$ & $\begin{array}{l}\text { Total } \\
\text { water }\end{array}$ \\
\hline \multicolumn{4}{|l|}{ Lake Cumberland 05130103} \\
\hline Casey County, Kentucky & 0.005 & 0.00 & 0.005 \\
\hline Clinton County, Kentucky & 0.003 & 0.00 & 0.003 \\
\hline Laurel County, Kentucky & $<0.001$ & 0.00 & $<0.001$ \\
\hline Lincoln County, Kentucky & 0.018 & 0.00 & 0.018 \\
\hline McCreary County, Kentucky & 0.003 & 0.00 & 0.003 \\
\hline Pulaski County, Kentucky & 0.200 & 0.00 & 0.200 \\
\hline Rockcastle County, Kentucky & 0.001 & 0.00 & 0.001 \\
\hline Russell County, Kentucky & 0.051 & 0.00 & 0.051 \\
\hline Wayne County, Kentucky & 0.013 & 0.00 & 0.013 \\
\hline Pickett County, Tennessee & ${ }^{1} 0.00$ & ${ }^{1} 0.00$ & 0.00 \\
\hline Lake Cumberland total & 0.294 & 0.00 & 0.294 \\
\hline \multicolumn{4}{|l|}{ South Fork Cumberland 05130104} \\
\hline McCreary County, Kentucky & 0.020 & 0.00 & 0.020 \\
\hline Pulaski County, Kentucky & 0.011 & 0.00 & 0.011 \\
\hline Wayne County, Kentucky & 0.006 & 0.00 & 0.006 \\
\hline Anderson County, Tennessee & 0.002 & 0.009 & 0.011 \\
\hline Scott County, Tennessee & ${ }^{1} 0.00$ & ${ }^{1} 0.00$ & 0.00 \\
\hline Campbell County, Tennessee & ${ }^{1} 0.00$ & ${ }^{1} 0.007$ & 0.007 \\
\hline Fentress County, Tennessee & 0.00 & 0.009 & 0.009 \\
\hline Pickett County, Tennessee & ${ }^{1} 0.00$ & ${ }^{1} 0.00$ & 0.00 \\
\hline South Fork Cumberland total & 0.040 & 0.025 & 0.064 \\
\hline Wolf Creek RCA total & 0.423 & 0.051 & 0.474 \\
\hline \multicolumn{4}{|l|}{ Dale Hollow } \\
\hline \multicolumn{4}{|l|}{ Obey 05130105} \\
\hline Clinton County, Kentucky & 0.006 & 0.00 & 0.006 \\
\hline Cumberland County, Kentucky & ${ }^{1} 0.001$ & ${ }^{1} 0.00$ & 0.001 \\
\hline Wayne County, Kentucky & $<0.001$ & 0.00 & $<0.001$ \\
\hline Clay County, Tennessee & ${ }^{1} 0.00$ & ${ }^{1} 0.006$ & 0.006 \\
\hline Cumberland County, Tennessee & 0.00 & 0.005 & 0.005 \\
\hline Fentress County, Tennessee & 0.00 & 0.010 & 0.010 \\
\hline Overton County, Tennessee & 0.00 & 0.064 & 0.064 \\
\hline Pickett County, Tennessee & ${ }^{1} 0.00$ & ${ }^{1} 0.00$ & 0.00 \\
\hline Putnam County, Tennessee & 0.00 & 0.006 & 0.006 \\
\hline Obey total & 0.007 & 0.092 & 0.099 \\
\hline Dale Hollow RCA total & 0.007 & 0.092 & 0.099 \\
\hline
\end{tabular}


Table 7. Irrigation water withdrawals by reservoir catchment area, hydrologic unit, and county, Cumberland River watershed, 2010.-Continued

[Values may not add to totals because of independent rounding. All values in million gallons per day; <, less than; $<0.001$, actual values less than 0.001 not in table but used to sum totals. Areal extent of all reservoir catchment areas is less than the areal extent of the Cumberland River watershed. RCA, reservoir catchment area]

\begin{tabular}{|c|c|c|c|}
\hline $\begin{array}{l}\text { RCA } \\
\text { Hydrologic unit } \\
\text { County, State }\end{array}$ & $\begin{array}{c}\text { Surface-water } \\
\text { withdrawal }\end{array}$ & $\begin{array}{l}\text { Groundwater } \\
\text { withdrawal }\end{array}$ & $\begin{array}{l}\text { Total } \\
\text { water }\end{array}$ \\
\hline \multicolumn{4}{|l|}{ Cordell Hull } \\
\hline \multicolumn{4}{|l|}{ Lake Cumberland 05130103} \\
\hline Adair County, Kentucky & 0.003 & 0.00 & 0.003 \\
\hline Clinton County, Kentucky & 0.001 & 0.00 & 0.001 \\
\hline Cumberland County, Kentucky & ${ }^{1} 0.009$ & ${ }^{1} 0.00$ & 0.009 \\
\hline Metcalfe County, Kentucky & 0.003 & 0.00 & 0.003 \\
\hline Monroe County, Kentucky & 0.013 & 0.00 & 0.013 \\
\hline Russell County, Kentucky & 0.016 & 0.00 & 0.016 \\
\hline Clay County, Tennessee & ${ }^{1} 0.00$ & ${ }^{1} 0.003$ & 0.003 \\
\hline Lake Cumberland total & 0.045 & 0.003 & 0.047 \\
\hline \multicolumn{4}{|l|}{ Cordell Hull 05130106} \\
\hline Clay County, Tennessee & ${ }^{1} 0.00$ & ${ }^{1} 0.007$ & 0.007 \\
\hline Jackson County, Tennessee & 0.00 & 0.060 & 0.060 \\
\hline Macon County, Tennessee & 0.00 & 0.003 & 0.003 \\
\hline Overton County, Tennessee & 0.00 & 0.046 & 0.046 \\
\hline Putnam County, Tennessee & 0.00 & 0.019 & 0.019 \\
\hline Smith County, Tennessee & 0.00 & 0.035 & 0.035 \\
\hline Cordell Hull total & 0.00 & 0.169 & 0.169 \\
\hline Cordell Hull RCA total & 0.045 & 0.172 & 0.217 \\
\hline \multicolumn{4}{|l|}{ Center Hill } \\
\hline \multicolumn{4}{|l|}{ Collins 05130107} \\
\hline Cannon County, Tennessee & 0.00 & 0.021 & 0.021 \\
\hline Coffee County, Tennessee & ${ }^{1} 0.021$ & ${ }^{1} 0.178$ & 0.199 \\
\hline Grundy County, Tennessee & 0.00 & 0.283 & 0.283 \\
\hline Sequatchie County, Tennessee & 0.00 & 0.001 & 0.001 \\
\hline Van Buren County, Tennessee & ${ }^{1} 0.00$ & ${ }^{1} 0.00$ & 0.00 \\
\hline Warren County, Tennessee & 0.680 & 3.73 & 4.41 \\
\hline Collins total & 0.701 & 4.21 & 4.91 \\
\hline \multicolumn{4}{|l|}{ Caney 05130108} \\
\hline Bledsoe County, Tennessee & 0.00 & 0.139 & 0.139 \\
\hline Cannon County, Tennessee & 0.00 & $<0.001$ & $<0.001$ \\
\hline Cumberland County, Tennessee & 0.00 & 0.058 & 0.058 \\
\hline Putnam County, Tennessee & 0.00 & 0.038 & 0.038 \\
\hline Sequatchie County, Tennessee & 0.00 & $<0.001$ & $<0.001$ \\
\hline
\end{tabular}


Table 7. Irrigation water withdrawals by reservoir catchment area, hydrologic unit, and county, Cumberland River watershed, 2010.-Continued

[Values may not add to totals because of independent rounding. All values in million gallons per day; <, less than; $<0.001$, actual values less than 0.001 not in table but used to sum totals. Areal extent of all reservoir catchment areas is less than the areal extent of the Cumberland River watershed. RCA, reservoir catchment area]

\begin{tabular}{|c|c|c|c|}
\hline $\begin{array}{l}\text { RCA } \\
\text { Hydrologic unit } \\
\text { County, State }\end{array}$ & $\begin{array}{c}\text { Surface-water } \\
\text { withdrawal }\end{array}$ & $\begin{array}{l}\text { Groundwater } \\
\text { withdrawal }\end{array}$ & $\begin{array}{c}\text { Total } \\
\text { water }\end{array}$ \\
\hline Van Buren County, Tennessee & ${ }^{1} 0.00$ & ${ }^{1} 0.00$ & 0.00 \\
\hline Warren County, Tennessee & 0.070 & 0.384 & 0.454 \\
\hline White County, Tennessee & 0.00 & 0.040 & 0.040 \\
\hline Caney total & 0.070 & 0.660 & 0.730 \\
\hline Center Hill RCA total & 0.771 & 4.87 & 5.64 \\
\hline \multicolumn{4}{|l|}{ Old Hickory } \\
\hline \multicolumn{4}{|l|}{ Cordell Hull 05130106} \\
\hline Smith County, Tennessee & 0.00 & 0.003 & 0.003 \\
\hline Cordell Hull total & 0.00 & 0.003 & 0.003 \\
\hline \multicolumn{4}{|l|}{ Caney 05130108} \\
\hline Cannon County, Tennessee & 0.00 & 0.020 & 0.020 \\
\hline Putnam County, Tennessee & 0.00 & 0.007 & 0.007 \\
\hline Rutherford County, Tennessee & 0.00 & $<0.001$ & $<0.001$ \\
\hline Smith County, Tennessee & 0.00 & 0.059 & 0.059 \\
\hline Wilson County, Tennessee & 0.00 & 0.008 & 0.008 \\
\hline Caney total & 0.00 & 0.095 & 0.095 \\
\hline \multicolumn{4}{|l|}{ Old Hickory Lake 05130201} \\
\hline Davidson County, Tennessee & $<0.001$ & 0.003 & 0.003 \\
\hline Macon County, Tennessee & 0.00 & 0.016 & 0.016 \\
\hline Robertson County, Tennessee & 0.00 & $<0.001$ & $<0.001$ \\
\hline Smith County, Tennessee & 0.00 & 0.063 & 0.063 \\
\hline Sumner County, Tennessee & 0.00 & 0.110 & 0.110 \\
\hline Trousdale County, Tennessee & 0.00 & 0.090 & 0.090 \\
\hline Wilson County, Tennessee & 0.00 & 0.048 & 0.048 \\
\hline Old Hickory Lake total & 0.00 & 0.331 & 0.331 \\
\hline Old Hickory RCA total & $<0.001$ & 0.429 & 0.430 \\
\hline
\end{tabular}

\section{J. Percy Priest}

Stones 05130203

\begin{tabular}{lccc} 
Cannon County, Tennessee & 0.00 & 0.039 & 0.039 \\
Coffee County, Tennessee & $1<0.001$ & 10.001 & 0.002 \\
Davidson County, Tennessee & 0.003 & 0.029 & 0.032 \\
Rutherford County, Tennessee & 0.00 & 0.881 & 0.881 \\
Williamson County, Tennessee & $<0.001$ & 0.001 & 0.001 \\
Wilson County, Tennessee & 0.00 & 0.021 & 0.021 \\
\hline Stones total & 0.003 & 0.973 & 0.976
\end{tabular}


Table 7. Irrigation water withdrawals by reservoir catchment area, hydrologic unit, and county, Cumberland River watershed, 2010._-Continued

[Values may not add to totals because of independent rounding. All values in million gallons per day; $<$, less than; $<0.001$, actual values less than 0.001 not in table but used to sum totals. Areal extent of all reservoir catchment areas is less than the areal extent of the Cumberland River watershed. RCA, reservoir catchment area]

\begin{tabular}{lccc}
\hline $\begin{array}{l}\text { RCA } \\
\begin{array}{l}\text { Hydrologic unit } \\
\text { County, State }\end{array}\end{array}$ & $\begin{array}{c}\text { Surface-water } \\
\text { withdrawal }\end{array}$ & $\begin{array}{c}\text { Groundwater } \\
\text { withdrawal }\end{array}$ & $\begin{array}{c}\text { Total } \\
\text { water }\end{array}$ \\
\hline J. Percy Priest RCA total & $\mathbf{0 . 0 0 3}$ & $\mathbf{0 . 9 7 3}$ & $\mathbf{0 . 9 7 6}$
\end{tabular}

\section{Cheatham}

Sycamore 05130202

Cheatham County, Tennessee

$0.00 \quad 0.128 \quad 0.128$

Davidson County, Tennessee

0.022

0.220

0.242

Dickson County, Tennessee

$<0.001$

$<0.001$

$<0.001$

Robertson County, Tennessee

0.00

0.128

0.128

Rutherford County, Tennessee

0.00

0.001

0.001

Sumner County, Tennessee

0.00

0.010

0.010

Williamson County, Tennessee

0.006

0.031

0.037

Sycamore total

0.028

0.519

0.547

Stones 05130203

\begin{tabular}{clll} 
Davidson County, Tennessee & 0.001 & 0.015 & 0.016 \\
Wilson County, Tennessee & 0.00 & 0.002 & 0.002 \\
\hline Stones total & 0.001 & 0.017 & 0.019
\end{tabular}

Harpeth 05130204

Cheatham County, Tennessee

$\begin{array}{lll}0.00 & 0.073 & 0.073 \\ 0.003 & 0.033 & 0.036 \\ 0.025 & 0.050 & 0.075 \\ 0.00 & 0.001 & 0.001 \\ 0.00 & 0.084 & 0.084 \\ 0.078 & 0.396 & 0.474 \\ 0.106 & 0.635 & 0.741 \\ 0.135 & \mathbf{1 . 1 7} & \mathbf{1 . 3 1}\end{array}$

Cheatham RCA total

0.135

$\mathbf{1 . 1 7}$

1.31

Barkley

Lower Cumberland 05130205

Caldwell County, Kentucky

Christian County, Kentucky

0.373

0.013

0.386

Crittenden County, Kentucky

0.587

0.018

0.604

Livingston County, Kentucky

0.023

0.00

0.023

0.030

0.00

0.030

Lyon County, Kentucky

${ }^{1} 0.00$

${ }^{1} 0.00$

0.00

Todd County, Kentucky

0.001

$<0.001$

0.001

Trigg County, Kentucky

0.634

0.009

0.643

Cheatham County, Tennessee

0.00

0.048

0.048

Dickson County, Tennessee

0.024

0.049

0.073 
Table 7. Irrigation water withdrawals by reservoir catchment area, hydrologic unit, and county, Cumberland River watershed, 2010.-Continued

[Values may not add to totals because of independent rounding. All values in million gallons per day; $<$, less than; $<0.001$, actual values less than 0.001 not in table but used to sum totals. Areal extent of all reservoir catchment areas is less than the areal extent of the Cumberland River watershed. RCA, reservoir catchment area]

\begin{tabular}{|c|c|c|c|}
\hline $\begin{array}{l}\text { RCA } \\
\text { Hydrologic unit } \\
\text { County, State }\end{array}$ & $\begin{array}{l}\text { Surface-water } \\
\text { withdrawal }\end{array}$ & $\begin{array}{l}\text { Groundwater } \\
\text { withdrawal }\end{array}$ & $\begin{array}{c}\text { Total } \\
\text { water }\end{array}$ \\
\hline Houston County, Tennessee & ${ }^{1} 0.00$ & ${ }^{1} 0.010$ & 0.010 \\
\hline Montgomery County, Tennessee & 0.00 & 0.314 & 0.314 \\
\hline Robertson County, Tennessee & 0.00 & 0.002 & 0.002 \\
\hline Stewart County, Tennessee & 0.044 & 0.075 & 0.118 \\
\hline Lower Cumberland total & 1.71 & 0.538 & 2.25 \\
\hline \multicolumn{4}{|l|}{ Red 05130206} \\
\hline Christian County, Kentucky & 0.248 & 0.007 & 0.256 \\
\hline Logan County, Kentucky & 0.076 & 0.004 & 0.080 \\
\hline Simpson County, Kentucky & 0.013 & 0.00 & 0.013 \\
\hline Todd County, Kentucky & 0.211 & 0.006 & 0.217 \\
\hline Trigg County, Kentucky & 0.004 & $<0.001$ & 0.004 \\
\hline Cheatham County, Tennessee & 0.00 & 0.001 & 0.001 \\
\hline Davidson County, Tennessee & $<0.001$ & $<0.001$ & $<0.001$ \\
\hline Montgomery County, Tennessee & 0.00 & 0.236 & 0.236 \\
\hline Robertson County, Tennessee & 0.00 & 1.37 & 1.37 \\
\hline Stewart County, Tennessee & 0.005 & 0.009 & 0.014 \\
\hline Sumner County, Tennessee & 0.00 & 0.022 & 0.022 \\
\hline Red total & 0.556 & 1.66 & 2.21 \\
\hline Barkley RCA total & 2.27 & 2.19 & 4.46 \\
\hline RCA total & 3.66 & 9.95 & 13.6 \\
\hline Kentucky & 2.68 & 0.058 & 2.74 \\
\hline Tennessee & 0.985 & 9.89 & 10.9 \\
\hline Cumberland River watershed total & 3.66 & 9.95 & 13.6 \\
\hline
\end{tabular}

${ }^{1}$ Irrigation data from the 2005 U.S. Geological Survey national compilation. 


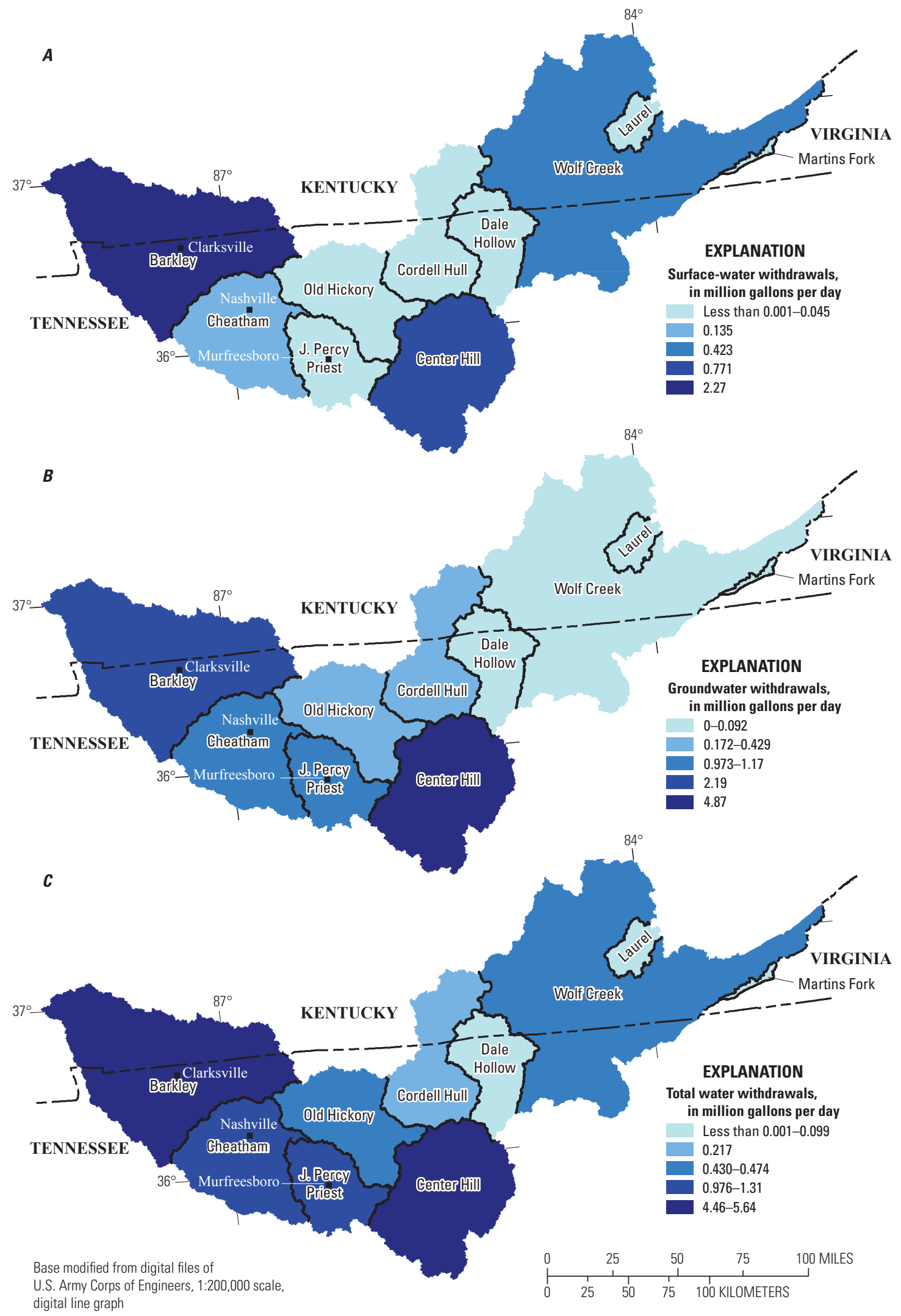

Figure 11. Irrigation water withdrawals by source, $(A)$ surface water, $(B)$ groundwater, and $(C)$ total water, and by reservoir catchment area in the Cumberland River watershed in 2010. 


\section{Public-Supply Water Projections for All Reservoir Catchment Areas to 2040}

From 2010 to 2040, the supply of raw and (or) finished water to meet demand for all 10 RCAs in the Cumberland River watershed is projected to increase from $360.10 \mathrm{Mgal} / \mathrm{d}$ to $532.51 \mathrm{Mgal} / \mathrm{d}$, or 48 percent (table 8; figs. 12 and 13). In the Barkley RCA, public-supply water withdrawals are projected to increase 41 percent, from $51.5 \mathrm{Mgal} / \mathrm{d}$ to $72.5 \mathrm{Mgal} / \mathrm{d}$. The Barkley RCA provides water to Clarksville, Tennessee, which is the second-largest population center in the study area. From 2010 to 2040, the combined total water demand for the Cheatham, J. Percy Priest, and Old Hickory RCAs is projected to increase from $224.08 \mathrm{Mgal} / \mathrm{d}$ to $359.58 \mathrm{Mgal} / \mathrm{d}$, or 61 percent (table 8). During 2010, 2020, 2030, and 2040, the combined projected demands in the Cheatham, J. Percy Priest, and Old Hickory RCAs are between 62 and 68 percent of the total water demands during the same periods. From 2010 to 2040, the combined total water demand for the Center Hill, Cordell Hull, and Dale Hollow RCAs is projected to increase from $31.7 \mathrm{Mgal} / \mathrm{d}$ to $43.0 \mathrm{Mgal} / \mathrm{d}$, or 36 percent. During 2010, 2020, 2030, and 2040, the combined projected demands in the Center Hill, Cordell Hull, and Dale Hollow RCAs are between 8 and 9 percent of the total water demands during the same periods. From 2010 to 2040, the combined total water demand for the Martins Fork, Laurel, and Wolf Creek RCAs is projected to increase from $52.8 \mathrm{Mgal} / \mathrm{d}$ to $57.4 \mathrm{Mgal} / \mathrm{d}$, or 9 percent (table 8). During 2010, 2020, 2030, and 2040, the combined projected demands in the Martins Fork, Laurel, and Wolf Creek RCAs are between 11 and 15 percent of the total water demands during the same periods. During 2010 to 2040, the Martins Fork RCA is the only RCA in the Cumberland River watershed with a projected decrease in water use (fig. 13; table 9).

Table 8. Public-supply water use, 2010, and projections of public-supply water use, 2020-2040, by reservoir catchment area, Cumberland River watershed.

[Values may not add to totals because of independent rounding. All values in million gallons per day]

\begin{tabular}{lrcccc}
\hline \multirow{5}{*}{$\begin{array}{c}\text { Reservoir } \\
\text { catchment } \\
\text { area }\end{array}$} & $\begin{array}{c}\text { Area } \\
\text { (square } \\
\text { miles) }\end{array}$ & $\begin{array}{c}\text { Bupply of raw and (or) finished water } \\
\text { to meet internal demand }\end{array}$ \\
\cline { 3 - 6 } & & $\begin{array}{c}\text { Bear } \\
\mathbf{2 0 1 0}\end{array}$ & $\mathbf{2 0 2 0}$ & $\mathbf{2 0 3 0}$ & $\mathbf{2 0 4 0}$ \\
\hline Martins Fork & 56 & 2.30 & 2.05 & 1.77 & 1.51 \\
Laurel & 282 & 8.83 & 9.29 & 9.55 & 9.61 \\
Wolf Creek & 5,449 & 41.7 & 43.9 & 45.5 & 46.3 \\
Dale Hollow & 936 & 2.08 & 2.25 & 2.47 & 2.72 \\
Cordell Hull & 1,375 & 4.99 & 5.06 & 5.22 & 5.38 \\
Center Hill & 2,169 & 24.6 & 27.7 & 31.1 & 34.9 \\
Old Hickory & 1,405 & 41.86 & 49.72 & 58.38 & 66.71 \\
J. Percy Priest & 894 & 34.35 & 45.11 & 60.96 & 78.90 \\
Cheatham & 1,595 & 147.87 & 170.29 & 191.77 & 213.96 \\
Barkley & 3,433 & 51.5 & 59.7 & 66.3 & 72.5 \\
\hline Total & $\mathbf{1 7 , 5 9 3}$ & $\mathbf{3 6 0 . 1 0}$ & $\mathbf{4 1 5 . 0 0}$ & $\mathbf{4 7 3 . 0 3}$ & $\mathbf{5 3 2 . 5 1}$ \\
\hline
\end{tabular}

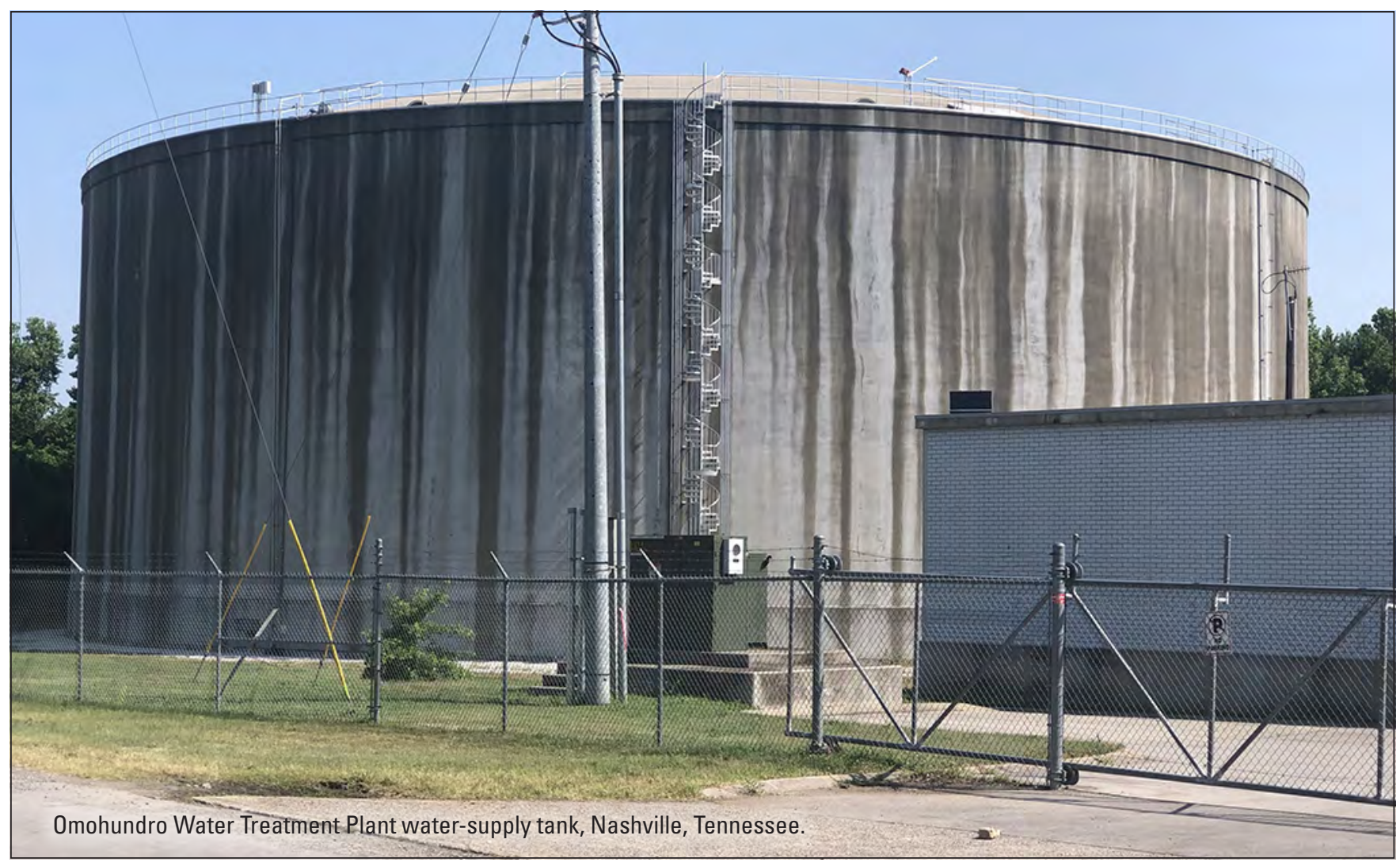



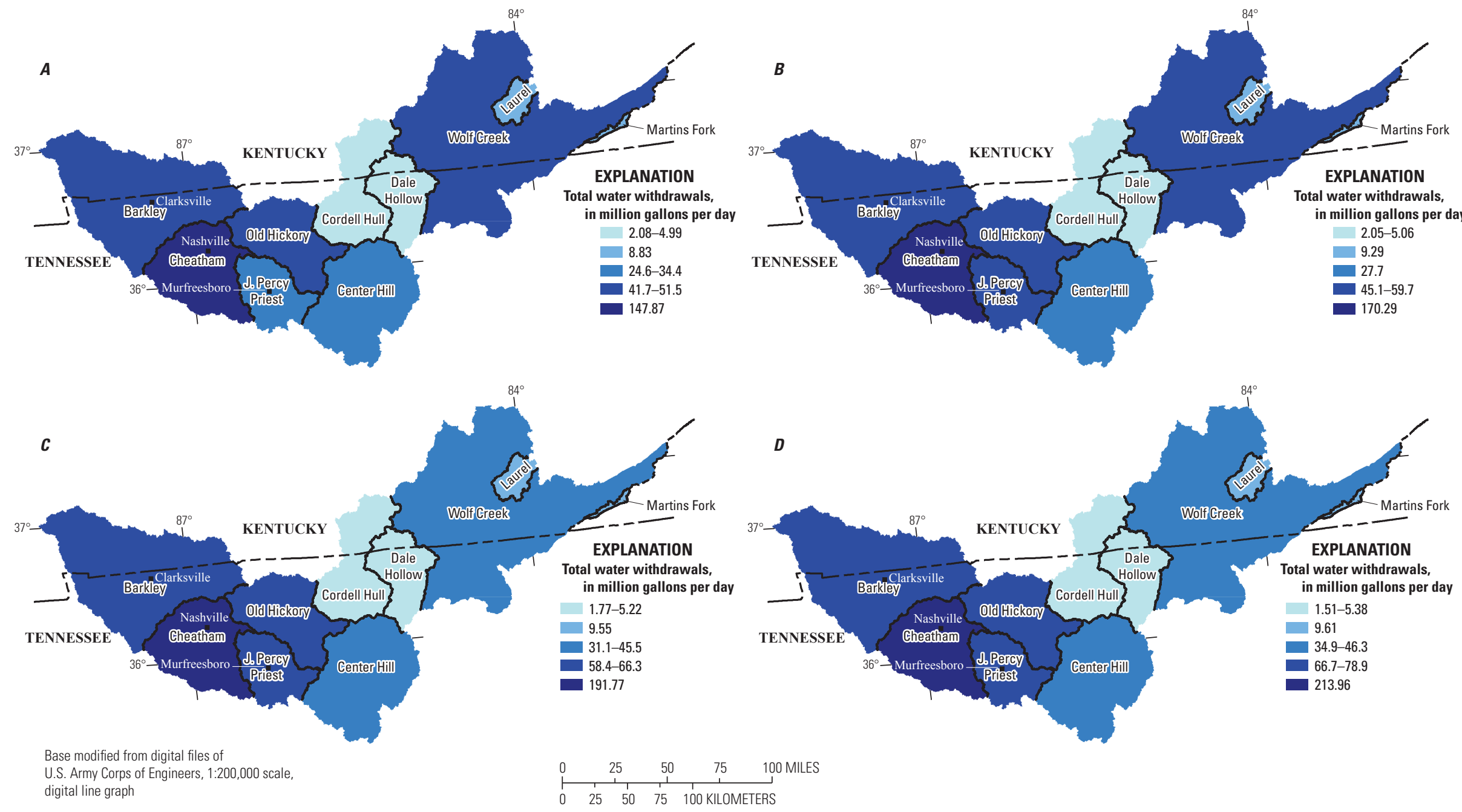

Base modified from digital files of

\section{Total waPANATION}

in million gallons per d

1.77-5.2

$31.1-45.5$

- $58.4-66.3$

$58.4-66.3$
-191.77
Figure 12. Public-supply water use for $(A) 2010$ and projections of public-supply water withdrawals for $(B) 2020,(C) 2030$, and $(D) 2040$ by reservoir catchment area in the Cumberland River watershed.

U.S. Army Corps of Engineers, 1:200,000 scale,
digital line graph

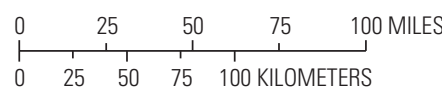

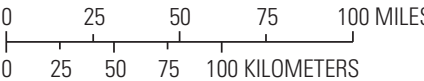




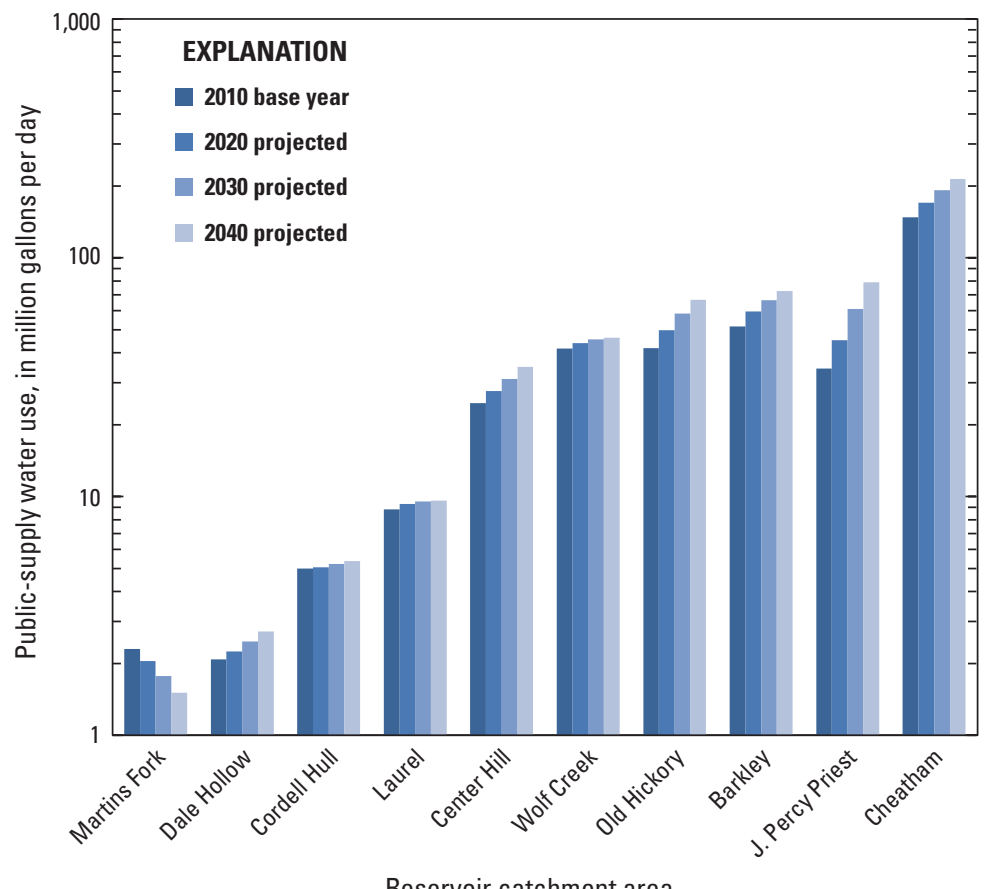

Figure 13. Public-supply water use by reservoir catchment area in the Cumberland River watershed, 2010 to 2040.

Table 9. Public-supply water use, 2010, and projections of public-supply water use, 2020-2040, by reservoir catchment area, hydrologic unit, and county, Cumberland River watershed.

[Values may not add to totals because of independent rounding. All values in million gallons per day. RCA, reservoir catchment area. Areal extent of all reservoir catchment areas is less than the areal extent of the Cumberland River watershed]

\begin{tabular}{|c|c|c|c|c|}
\hline \multirow{2}{*}{$\begin{array}{l}\text { RCA } \\
\text { Hydrologic unit } \\
\text { County, State }\end{array}$} & \multicolumn{4}{|c|}{ Supply of raw and (or) finished water to meet internal demand } \\
\hline & $\begin{array}{c}\text { Base year } \\
2010\end{array}$ & 2020 & 2030 & 2040 \\
\hline \multicolumn{5}{|l|}{ Martins Fork } \\
\hline \multicolumn{5}{|l|}{ Upper Cumberland 05130101} \\
\hline Harlan County, Kentucky & 2.30 & 2.05 & 1.77 & 1.51 \\
\hline Upper Cumberland total & 2.30 & 2.05 & 1.77 & 1.51 \\
\hline Martins Fork RCA total & 2.30 & 2.05 & 1.77 & 1.51 \\
\hline \multicolumn{5}{|l|}{ Laurel } \\
\hline \multicolumn{5}{|l|}{ Upper Cumberland 05130101} \\
\hline Laurel County, Kentucky & 5.01 & 5.51 & 5.91 & 6.20 \\
\hline Whitley County, Kentucky & 3.82 & 3.78 & 3.64 & 3.41 \\
\hline Upper Cumberland total & 8.83 & 9.29 & 9.55 & 9.61 \\
\hline Laurel RCA total & 8.83 & 9.29 & 9.55 & 9.61 \\
\hline
\end{tabular}


Table 9. Public-supply water use, 2010, and projections of public-supply water use, 2020-2040, by reservoir catchment area, hydrologic unit, and county, Cumberland River watershed._Continued

[Values may not add to totals because of independent rounding. All values in million gallons per day. RCA, reservoir catchment area. Areal extent of all reservoir catchment areas is less than the areal extent of the Cumberland River watershed]

\begin{tabular}{|c|c|c|c|c|}
\hline \multirow{2}{*}{$\begin{array}{l}\text { RCA } \\
\text { Hydrologic unit } \\
\text { County, State }\end{array}$} & \multicolumn{4}{|c|}{ Supply of raw and (or) finished water to meet internal demand } \\
\hline & $\begin{array}{c}\text { Base year } \\
2010\end{array}$ & 2020 & 2030 & 2040 \\
\hline \multicolumn{5}{|l|}{ Wolf Creek } \\
\hline \multicolumn{5}{|l|}{ Upper Cumberland 05130101} \\
\hline Bell County, Kentucky & 3.84 & 3.70 & 3.47 & 3.21 \\
\hline Harlan County, Kentucky & 1.36 & 1.21 & 1.05 & 0.89 \\
\hline Knox County, Kentucky & 0.326 & 0.329 & 0.324 & 0.312 \\
\hline McCreary County, Kentucky & 1.17 & 1.14 & 1.09 & 1.02 \\
\hline Whitley County, Kentucky & 1.55 & 1.53 & 1.47 & 1.38 \\
\hline Campbell County, Tennessee & 0.954 & 1.01 & 1.06 & 1.11 \\
\hline Claiborne County, Tennessee & 0.151 & 0.157 & 0.164 & 0.169 \\
\hline Upper Cumberland total & 9.34 & 9.07 & 8.63 & 8.09 \\
\hline \multicolumn{5}{|l|}{ Rockcastle 05130102} \\
\hline Jackson County, Kentucky & 1.80 & 1.84 & 1.80 & 1.72 \\
\hline Laurel County, Kentucky & 4.09 & 4.50 & 4.83 & 5.06 \\
\hline Rockcastle County, Kentucky & 2.34 & 2.41 & 2.43 & 2.38 \\
\hline Rockcastle total & 8.23 & 8.75 & 9.06 & 9.15 \\
\hline \multicolumn{5}{|l|}{ Lake Cumberland 05130103} \\
\hline Clinton County, Kentucky & 3.40 & 3.61 & 3.73 & 3.80 \\
\hline Lincoln County, Kentucky & 0.277 & 0.293 & 0.302 & 0.304 \\
\hline Pulaski County, Kentucky & 9.04 & 10.2 & 11.2 & 12.2 \\
\hline Russell County, Kentucky & 3.48 & 3.72 & 3.90 & 4.02 \\
\hline Wayne County, Kentucky & 2.46 & 2.62 & 2.69 & 2.66 \\
\hline Lake Cumberland total & 18.7 & 20.4 & 21.9 & 22.9 \\
\hline \multicolumn{5}{|l|}{ South Fork Cumberland 05130104} \\
\hline McCreary County, Kentucky & 0.722 & 0.722 & 0.702 & 0.667 \\
\hline Pulaski County, Kentucky & 0.054 & 0.061 & 0.067 & 0.072 \\
\hline Fentress County, Tennessee & 1.74 & 1.87 & 2.02 & 2.18 \\
\hline Scott County, Tennessee & 2.50 & 2.48 & 2.60 & 2.72 \\
\hline Morgan County, Tennessee & 0.467 & 0.490 & 0.508 & 0.518 \\
\hline South Fork Cumberland total & 5.48 & 5.63 & 5.89 & 6.15 \\
\hline Wolf Creek RCA total & 41.7 & 43.9 & 45.5 & 46.3 \\
\hline
\end{tabular}


Table 9. Public-supply water use, 2010, and projections of public-supply water use, 2020-2040, by reservoir catchment area, hydrologic unit, and county, Cumberland River watershed._-Continued

[Values may not add to totals because of independent rounding. All values in million gallons per day. RCA, reservoir catchment area. Areal extent of all reservoir catchment areas is less than the areal extent of the Cumberland River watershed]

\begin{tabular}{|c|c|c|c|c|}
\hline \multirow{2}{*}{$\begin{array}{l}\text { RCA } \\
\text { Hydrologic unit } \\
\text { County, State }\end{array}$} & \multicolumn{4}{|c|}{ Supply of raw and (or) finished water to meet internal demand } \\
\hline & $\begin{array}{c}\text { Base year } \\
2010\end{array}$ & 2020 & 2030 & 2040 \\
\hline \multicolumn{5}{|l|}{ Dale Hollow } \\
\hline \multicolumn{5}{|l|}{ Obey 05130105} \\
\hline Fentress County, Tennessee & 0.084 & 0.091 & 0.098 & 0.106 \\
\hline Overton County, Tennessee & 0.385 & 0.407 & 0.433 & 0.460 \\
\hline Pickett County, Tennessee & 0.535 & 0.489 & 0.488 & 0.493 \\
\hline Putnam County, Tennessee & 1.07 & 1.26 & 1.45 & 1.66 \\
\hline Obey total & 2.08 & 2.25 & 2.47 & 2.72 \\
\hline Dale Hollow RCA total & 2.08 & 2.25 & 2.47 & 2.72 \\
\hline \multicolumn{5}{|l|}{ Cordell Hull } \\
\hline \multicolumn{5}{|l|}{ Lake Cumberland 05130103} \\
\hline Cumberland County, Tennessee & 0.776 & 0.753 & 0.715 & 0.672 \\
\hline Lake Cumberland total & 0.776 & 0.753 & 0.715 & 0.672 \\
\hline \multicolumn{5}{|l|}{ Cordell Hull 05130106} \\
\hline Clay County, Tennessee & 0.466 & 0.457 & 0.471 & 0.487 \\
\hline Jackson County, Tennessee & 0.788 & 0.782 & 0.797 & 0.806 \\
\hline Overton County, Tennessee & 2.26 & 2.39 & 2.54 & 2.69 \\
\hline Cordell Hull total & 3.52 & 3.63 & 3.80 & 3.98 \\
\hline
\end{tabular}

Obey 05130105

\begin{tabular}{lllll}
\multicolumn{1}{c}{ Clay County, Tennessee } & 0.694 & 0.681 & 0.702 & 0.726 \\
\hline Obey total & 0.694 & 0.681 & 0.702 & 0.726 \\
Cordell Hull RCA total & $\mathbf{4 . 9 9}$ & $\mathbf{5 . 0 6}$ & $\mathbf{5 . 2 2}$ & $\mathbf{5 . 3 8}$
\end{tabular}

Center Hill

\begin{tabular}{lcccc}
$\begin{array}{l}\text { Collins } 05130107 \\
\text { Grundy County, Tennessee }\end{array}$ & 0.664 & 0.647 & 0.658 & 0.657 \\
Warren County, Tennessee & 5.24 & 5.48 & 5.84 & 6.17 \\
\hline Collins total & 5.90 & 6.13 & 6.50 & 6.82 \\
& & & \\
Caney 05130108 & & & \\
Bledsoe County, Tennessee & 0.310 & 0.343 & 0.375 & 0.402 \\
DeKalb County, Tennessee & 0.841 & 0.876 & 0.946 & 1.03 \\
Putnam County, Tennessee & 12.8 & 15.1 & 17.5 & 20.2 \\
Van Buren County, Tennessee & 1.01 & 1.00 & 1.02 & 1.03 \\
White County, Tennessee & 3.81 & 4.23 & 4.77 & 5.38 \\
\hline Caney total & 18.7 & 21.5 & 24.6 & 28.1 \\
Center Hill RCA total & $\mathbf{2 4 . 6}$ & $\mathbf{2 7 . 7}$ & $\mathbf{3 1 . 1}$ & $\mathbf{3 4 . 9}$
\end{tabular}


Table 9. Public-supply water use, 2010, and projections of public-supply water use, 2020-2040, by reservoir catchment area, hydrologic unit, and county, Cumberland River watershed._Continued

[Values may not add to totals because of independent rounding. All values in million gallons per day. RCA, reservoir catchment area. Areal extent of all reservoir catchment areas is less than the areal extent of the Cumberland River watershed]

\begin{tabular}{|c|c|c|c|c|}
\hline \multirow{2}{*}{$\begin{array}{l}\text { RCA } \\
\text { Hydrologic unit } \\
\text { County, State }\end{array}$} & \multicolumn{4}{|c|}{ Supply of raw and (or) finished water to meet internal demand } \\
\hline & $\begin{array}{c}\text { Base year } \\
2010\end{array}$ & 2020 & 2030 & 2040 \\
\hline \multicolumn{5}{|l|}{ Old Hickory } \\
\hline \multicolumn{5}{|l|}{ Caney 05130108} \\
\hline DeKalb County, Tennessee & 1.34 & 1.39 & 1.50 & 1.63 \\
\hline Smith County, Tennessee & 0.275 & 0.298 & 0.329 & 0.357 \\
\hline Caney total & 1.61 & 1.69 & 1.83 & 1.99 \\
\hline \multicolumn{5}{|l|}{ Old Hickory Lake 05130201} \\
\hline Davidson County, Tennessee & 0.471 & 0.522 & 0.557 & 0.587 \\
\hline Smith County, Tennessee & 1.60 & 1.73 & 1.90 & 2.05 \\
\hline Sumner County, Tennessee & 23.6 & 28.9 & 34.2 & 39.4 \\
\hline Trousdale County, Tennessee & 0.858 & 0.961 & 1.07 & 1.17 \\
\hline Wilson County, Tennessee & 13.8 & 15.9 & 18.8 & 21.5 \\
\hline Old Hickory Lake total & 40.2 & 48.0 & 56.6 & 64.7 \\
\hline Old Hickory RCA total & 41.9 & 49.7 & 58.4 & 66.7 \\
\hline \multicolumn{5}{|l|}{ J. Percy Priest } \\
\hline \multicolumn{5}{|l|}{ Stones 05130203} \\
\hline Cannon County, Tennessee & 1.16 & 1.24 & 1.31 & 1.37 \\
\hline Rutherford County, Tennessee & 31.5 & 41.7 & 57.1 & 74.5 \\
\hline Wilson County, Tennessee & 1.71 & 2.16 & 2.58 & 3.00 \\
\hline Stones total & 34.4 & 45.1 & 61.0 & 78.9 \\
\hline J. Percy Priest RCA total & 34.4 & 45.1 & 61.0 & 78.9 \\
\hline \multicolumn{5}{|l|}{ Cheatham } \\
\hline \multicolumn{5}{|l|}{ Sycamore 05130202} \\
\hline Cheatham County, Tennessee & 2.33 & 2.77 & 2.86 & 2.83 \\
\hline Davidson County, Tennessee & 117.86 & 130.54 & 140.08 & 149.00 \\
\hline Williamson County, Tennessee & 2.97 & 4.25 & 5.93 & 7.87 \\
\hline Sycamore total & 123.17 & 137.56 & 148.87 & 159.70 \\
\hline \multicolumn{5}{|l|}{ Harpeth 05130204} \\
\hline Cheatham County, Tennessee & 0.652 & 0.692 & 0.72 & 0.71 \\
\hline Dickson County, Tennessee & 4.91 & 5.21 & 5.44 & 5.47 \\
\hline Williamson County, Tennessee & 19.1 & 26.8 & 36.7 & 48.1 \\
\hline Harpeth total & 24.7 & 32.7 & 42.9 & 54.3 \\
\hline Cheatham RCA total & 147.87 & 170.29 & 191.77 & 213.96 \\
\hline
\end{tabular}


Table 9. Public-supply water use, 2010, and projections of public-supply water use, 2020-2040, by reservoir catchment area, hydrologic unit, and county, Cumberland River watershed.—Continued

[Values may not add to totals because of independent rounding. All values in million gallons per day. RCA, reservoir catchment area. Areal extent of all reservoir catchment areas is less than the areal extent of the Cumberland River watershed]

\begin{tabular}{|c|c|c|c|c|}
\hline \multirow{2}{*}{$\begin{array}{l}\text { RCA } \\
\text { Hydrologic unit } \\
\text { County, State }\end{array}$} & \multicolumn{4}{|c|}{ Supply of raw and (or) finished water to meet internal demand } \\
\hline & $\begin{array}{c}\text { Base year } \\
2010\end{array}$ & 2020 & 2030 & 2040 \\
\hline \multicolumn{5}{|l|}{ Barkley } \\
\hline \multicolumn{5}{|l|}{ Lower Cumberland 05130205} \\
\hline Lyon County, Kentucky & 2.79 & 2.86 & 2.82 & 2.72 \\
\hline Trigg County, Kentucky & 14.9 & 16.9 & 18.7 & 20.0 \\
\hline Dickson County, Tennessee & 0.036 & 0.039 & 0.04 & 0.04 \\
\hline Houston County, Tennessee & 0.972 & 0.975 & 0.980 & 0.977 \\
\hline Montgomery County, Tennessee & 16.7 & 20.7 & 23.8 & 27.4 \\
\hline Stewart County, Tennessee & 0.858 & 1.10 & 1.17 & 1.24 \\
\hline Lower Cumberland total & 36.3 & 42.5 & 47.5 & 52.3 \\
\hline \multicolumn{5}{|l|}{ Red 05130206} \\
\hline Todd County, Kentucky & 3.88 & 4.04 & 4.14 & 4.19 \\
\hline Montgomery County, Kentucky & 5.60 & 6.64 & 7.44 & 8.27 \\
\hline Robertson County, Kentucky & 5.68 & 6.48 & 7.23 & 7.73 \\
\hline Red total & 15.2 & 17.2 & 18.8 & 20.2 \\
\hline Barkley RCA total & 51.5 & 59.7 & 66.3 & 72.5 \\
\hline RCA total & 360.10 & 415.00 & 473.03 & 532.51 \\
\hline
\end{tabular}

Lower Cumberland 05130205

Livingston County, Kentucky ${ }^{1}$

Kentucky total

Tennessee total

Cumberland River watershed total
1.35

$\mathbf{7 0 . 8}$

290.68

361.44
1.33

75.1

341.23

416.33
1.28

78.0

396.27

474.31
1.18

79.5

454.19

533.70

${ }^{1}$ No reservoir catchment associated with withdrawal. 


\section{Martins Fork Reservoir Catchment Area}

Population and water withdrawals are projected to decline through 2040 in the Martins Fork RCA. During 2010, the population served by each public-supply water system ranged from about 3,000 to 8,000 people, and the total for all systems in the Martins Fork RCA was about 17,400 people. Raw water withdrawals by three principal suppliers in the Martins Fork RCA totaled 2.30 Mgal/d. Because population served is projected to decrease by about 6,000 people from 2010 to 2040, the supply of raw water and (or) finished water to meet internal demand in the Martins Fork RCA is projected to decrease by 34 percent (from 2.30 to $1.51 \mathrm{Mgal} / \mathrm{d}$; table 10; fig. 14). Forty-seven percent of this projected decrease is attributed to Harlan Municipal Water Works, which relies on the Poor Fork Cumberland River for supply. Thirty-six percent of the projected decrease is attributed to Cumberland Municipal Water Works, which also relies on the Poor Fork Cumberland River. Seventeen percent of the projected decrease is attributed to the Cawood Water District, which relies on Martins Fork Cumberland River.
Table 10. Population served, public-supply water use during 2010, and public-supply water-use projections in the Martins Fork reservoir catchment area, 2020, 2030, and 2040.

\begin{tabular}{lcc}
\hline Year & $\begin{array}{c}\text { Total } \\
\text { Population } \\
\text { served } \\
\text { (thousands) }\end{array}$ & $\begin{array}{c}\text { public- } \\
\text { supply } \\
\text { water use } \\
\text { (million } \\
\text { gallons } \\
\text { per day) }\end{array}$ \\
\hline 2010 & 17.4 & 2.30 \\
2020 & 15.5 & 2.05 \\
2030 & 13.4 & 1.77 \\
2040 & 11.4 & 1.51 \\
\hline Change from 2010 to 2040, in percent & -34 & -34 \\
\hline
\end{tabular}

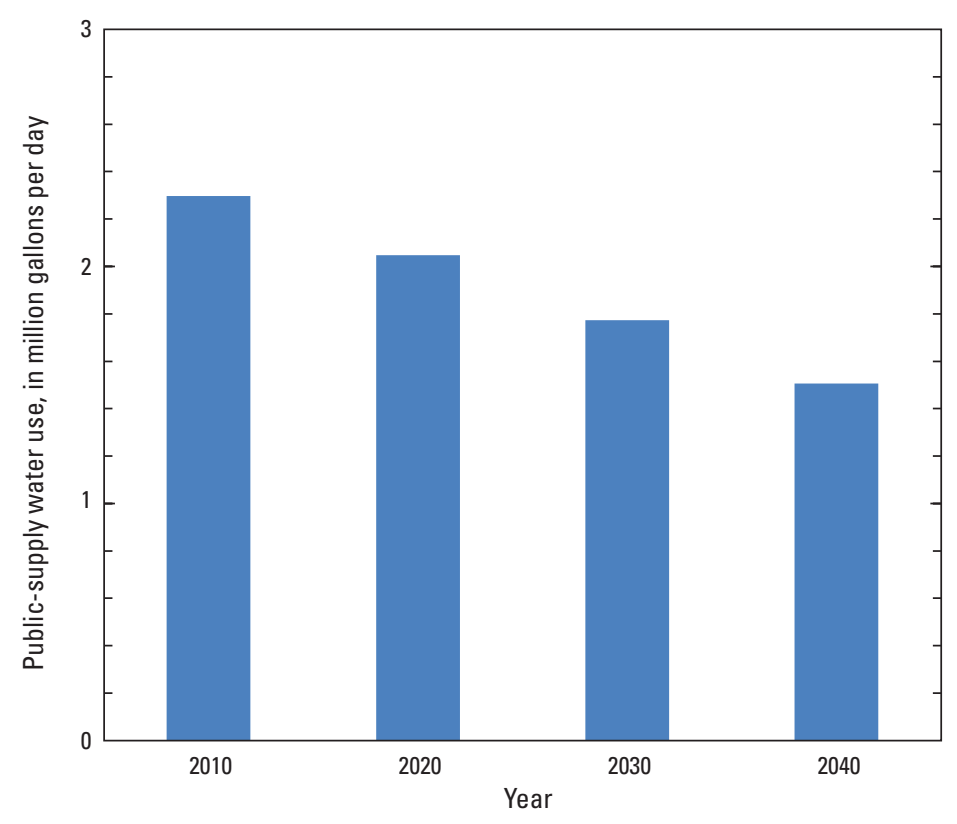

Figure 14. Public-supply water use in 2010 and projected public-supply water use for 2020-2040, Martins Fork reservoir catchment area, Cumberland River watershed. 


\section{Laurel Reservoir Catchment Area}

Population and water withdrawals are expected to increase through 2040 in the Laurel RCA. During 2010, the population served by each public-supply water system in the Laurel RCA ranged from about 8,000 to 24,000 people, and the total for all systems was about 55,000 people. Raw water withdrawals by four principal suppliers in the Laurel $\mathrm{RCA}$ totaled $8.83 \mathrm{Mgal} / \mathrm{d}$. Because population served is projected to increase by about 10,000 people from 2010 to 2040 , the supply of raw water and (or) finished water to meet internal demand in the Laurel RCA is projected to increase by 9 percent (from 8.83 to $9.61 \mathrm{Mgal} / \mathrm{d}$; table 11; fig. 15). An increase of $0.455 \mathrm{Mgal} / \mathrm{d}$ is expected to come from London Utility Commission, which relies on Laurel Lake for supply. Barbourville Water \& Electric is projected to provide $0.434 \mathrm{Mgal} / \mathrm{d}$ of the increase and relies on Poor Fork Cumberland River and Laurel River Lake. Laurel County Water District \#2 is projected to provide $0.295 \mathrm{Mgal} / \mathrm{d}$ of the increase and relies on Laurel River Lake and Laurel River. The withdrawals by Corbin City Utilities Commission are projected to decrease by $0.407 \mathrm{Mgal} / \mathrm{d}$, or 11 percent; the Laurel River is the source of these withdrawals. From 2010 to 2040, the percentage of increase in the supply of raw water and (or) finished water to meet internal demand for Barbourville Water \& Electric, Laurel County Water District \#2, and London Utility Commission in the Laurel RCA is 24 percent.
Table 11. Population served, public-supply water use during 2010 , and public-supply water-use projections in the Laurel reservoir catchment area, 2020, 2030, and 2040.

\begin{tabular}{lcc}
\hline Year & $\begin{array}{c}\text { Total } \\
\text { Population } \\
\text { served, } \\
\text { (thousands) }\end{array}$ & $\begin{array}{c}\text { public- } \\
\text { supply } \\
\text { water use } \\
\text { (million } \\
\text { gallons } \\
\text { per day) }\end{array}$ \\
\hline 2010 & 54.9 & 8.83 \\
2020 & 57.7 & 9.29 \\
2030 & 61.9 & 9.55 \\
2040 & 64.9 & 9.61 \\
\hline Change from 2010 to 2040, in percent & 18 & 9 \\
\hline
\end{tabular}

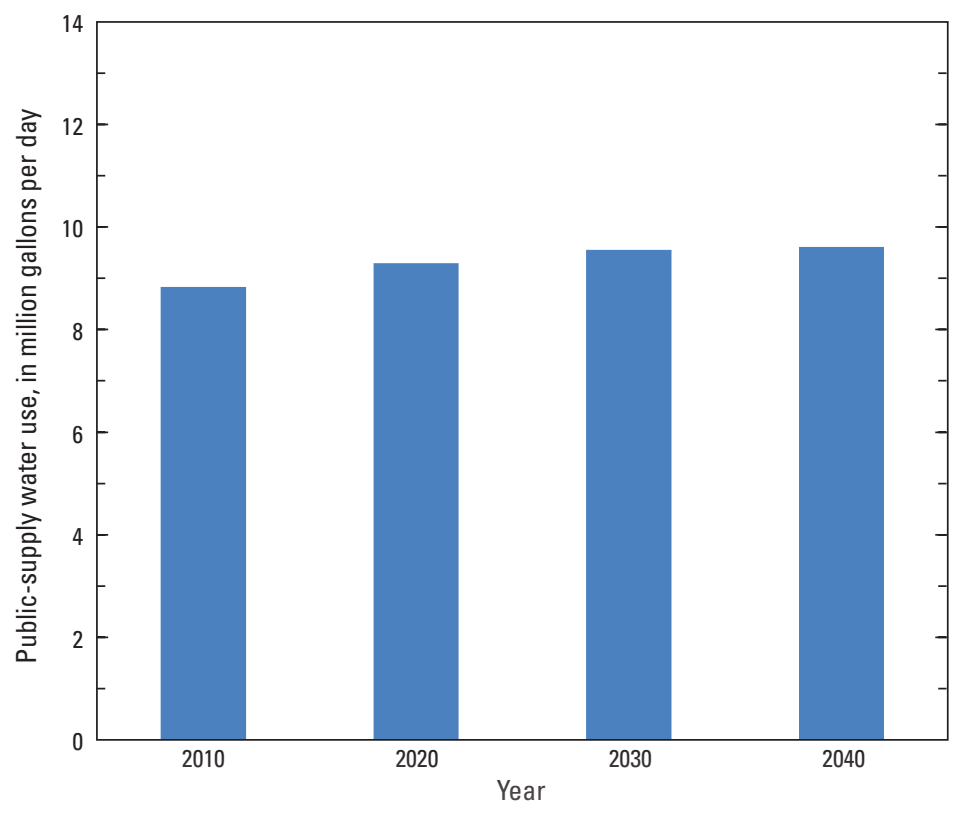

Figure 15. Public-supply water use in 2010 and projected publicsupply water use for 2020-2040, Laurel reservoir catchment area, Cumberland River watershed. 


\section{Wolf Creek Reservoir Catchment Area}

Population and water withdrawals are expected to increase through 2040 in the Wolf Creek RCA. During 2010, the population served by each system in the Wolf Creek RCA ranged from about 400 to 59,000 people, and the total for all systems was about 293,000 people. Raw water withdrawals by 26 principal suppliers in the Wolf Creek RCA totaled $41.3 \mathrm{Mgal} / \mathrm{d}$, ranging from 0.054 to $8.88 \mathrm{Mgal} / \mathrm{d}$. For eight systems in the Tennessee portion of the Wolf Creek RCA, residential use per capita ranged from 47 to 84 gallons per day. Nonrevenue water in the Tennessee portion of the Wolf Creek RCA totaled $2.73 \mathrm{Mgal} / \mathrm{d}$, which was 50 percent of the raw water withdrawals by principal suppliers in the Tennessee portion of the Wolf Creek RCA. The ratios of commercial and industrial to residential water use were determined for six water-supply systems and ranged from 0.003 to 0.479 .

Because population served is projected to increase by about 48,000 people from 2010 to 2040 , the supply of raw water and (or) finished water to meet internal demand in the Wolf Creek RCA is projected to increase by $4.63 \mathrm{Mgal} / \mathrm{d}$, or 11 percent (from 41.7 to $46.3 \mathrm{Mgal} / \mathrm{d}$; table 12; fig. 16). Somerset Water Service is projected to provide $3.06 \mathrm{Mgal} / \mathrm{d}$ of the increase and relies on Lake Cumberland. Wood Creek Water District, which relies on Wood Creek Lake, is projected to provide $0.967 \mathrm{Mgal} / \mathrm{d}$ of the increase. Jamestown Municipal Water Works is projected to provide $0.547 \mathrm{Mgal} / \mathrm{d}$ of the increase and relies on Lake Cumberland. Albany Water Works is projected to provide $0.397 \mathrm{Mgal} / \mathrm{d}$ of the increase and relies on Lake Cumberland. Other systems in the Wolf Creek RCA are projected to each supply from -0.392 to $0.547 \mathrm{Mgal} / \mathrm{d}$ of the total projected increase. From 2010 to 2040, the percentage of change in the supply of raw water and (or) finished water to meet internal demand for all systems in the Wolf Creek RCA is projected to range from -34 to 34 percent.
During 2010 to 2040, the projected increase in publicsupply water use is $0.882 \mathrm{Mgal} / \mathrm{d}$, or 15 percent, in the Tennessee portion of the Wolf Creek RCA. Of this projected increase, 46 percent of the total increase $(0.407 \mathrm{Mgal} / \mathrm{d})$ is projected to be delivered as finished water to residential customers, 6 percent $(0.051 \mathrm{Mgal} / \mathrm{d})$ is projected to be delivered as finished water to commercial and industrial customers, and the remainder ( 48 percent) is projected to be nonrevenue water. In the Tennessee portion of the Wolf Creek RCA, commercial and industrial water use represents 4 percent of the total finished water from 2010 through 2040.

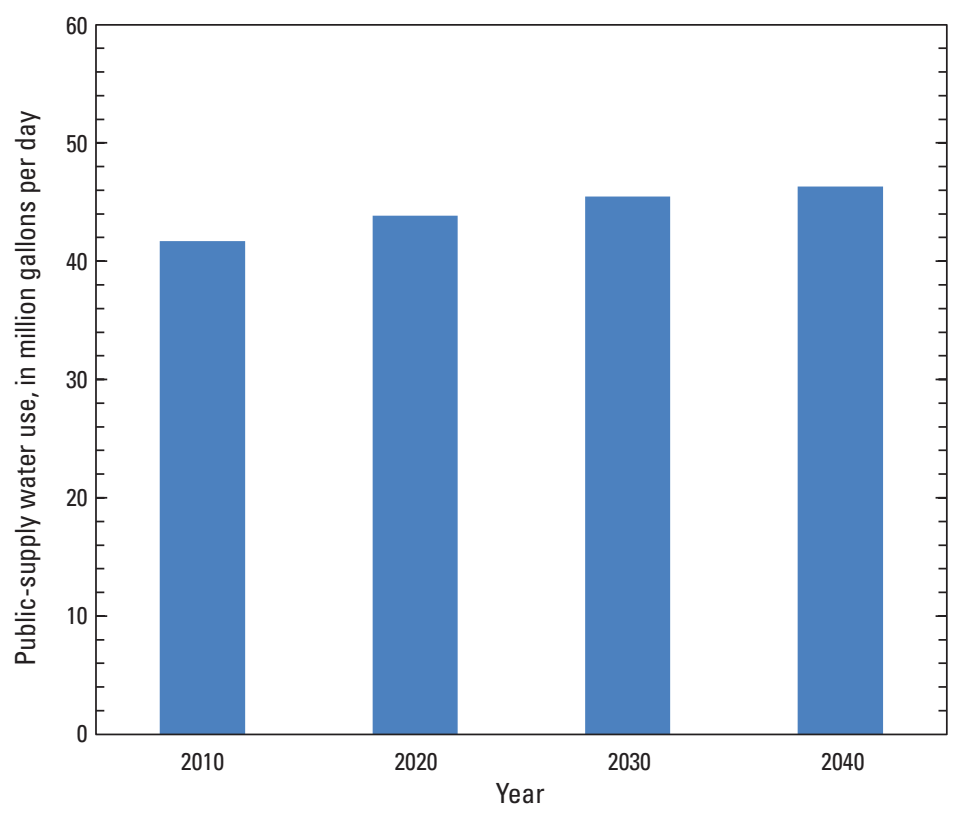

Figure 16. Public-supply water use in 2010 and projected public-supply water use for 2020-2040, Wolf Creek reservoir catchment area, Cumberland River watershed.

Table 12. Population served, public-supply water use during 2010, and public-supply water-use projections in the Wolf Creek reservoir catchment area, 2020, 2030, and 2040.

[Values in million gallons per day except where noted]

\begin{tabular}{|c|c|c|c|c|c|}
\hline \multirow[b]{2}{*}{ Year } & \multirow{2}{*}{$\begin{array}{c}\text { Population } \\
\text { served }^{1} \\
\text { (thousands) }\end{array}$} & \multicolumn{4}{|c|}{ Public-supply water use } \\
\hline & & Residential $^{2}$ & $\begin{array}{c}\text { Commercial } \\
\text { and industrial }{ }^{2}\end{array}$ & $\begin{array}{c}\text { Treatment } \\
\text { and nonrevenue }{ }^{2}\end{array}$ & Total' \\
\hline 2020 & 306 & 2.94 & 0.230 & 2.84 & 43.9 \\
\hline 2030 & 323 & 3.10 & 0.248 & 3.01 & 45.5 \\
\hline
\end{tabular}

${ }^{1}$ Values for the Tennessee and Kentucky portion of the Wolf Creek reservoir catchment area.

${ }^{2}$ Values for the Tennessee portion of the Wolf Creek reservoir catchment area. 


\section{Dale Hollow Reservoir Catchment Area}

Population and water withdrawals are expected to increase through 2040 in the Dale Hollow RCA. During 2010, the population served by each system in the Dale Hollow RCA ranged from about 30 to 5,100 people, and the total for all systems was about 15,000 people. Raw water withdrawals by three principal suppliers in the Dale Hollow RCA totaled $1.90 \mathrm{Mgal} / \mathrm{d}$. Residential use per capita ranged from 51 to 70 gallons per day. The ratios of commercial and industrial to residential water use were determined for five watersupply systems in the Dale Hollow RCA and ranged from 0.014 to 1.69 . Nonrevenue water in the Dale Hollow RCA totaled $0.653 \mathrm{Mgal} / \mathrm{d}$, which was 34 percent of the raw water withdrawals by principal suppliers.

Because population served is projected to increase by about 3,500 people from 2010 to 2040, the supply of raw water and (or) finished water to meet internal demand in the Dale Hollow RCA is projected to increase by 31 percent (from 2.08 to $2.72 \mathrm{Mgal} / \mathrm{d}$; table 13; fig. 17). Of this projected increase, 31 percent $(0.202 \mathrm{Mgal} / \mathrm{d})$ is projected to be delivered as finished water to residential customers, 25 percent $(0.163 \mathrm{Mgal} / \mathrm{d})$ as finished water to commercial and industrial customers, and the remainder (44 percent, or $0.282 \mathrm{Mgal} / \mathrm{d}$ ) as nonrevenue water. Monterey Water Department, which relies on City Lake/Meadow Creek Lake for supply, is to provide $0.590 \mathrm{Mgal} / \mathrm{d}$ of the projected increase. Heritage Academy is projected to provide $0.004 \mathrm{Mgal} / \mathrm{d}$ of the increase and relies on groundwater from a well. A projected decrease in water use of $0.042 \mathrm{Mgal} / \mathrm{d}$ is attributed to Byrdstown Water Department, which relies on the Obey River for supply. From 2010 to 2040, the percentage of change in the supply of raw water and (or) finished water to meet internal demand for all systems in the Dale Hollow RCA is projected to range from -8 to 35 percent.
In the Dale Hollow RCA, commercial and industrial water use represented 22 percent $(0.452 \mathrm{Mgal} / \mathrm{d})$ of the total finished water in 2010. By 2040, commercial and industrial water use is projected to be 23 percent $(0.614 \mathrm{Mgal} / \mathrm{d})$ of the total finished water, which is a 36-percent increase from 2010.

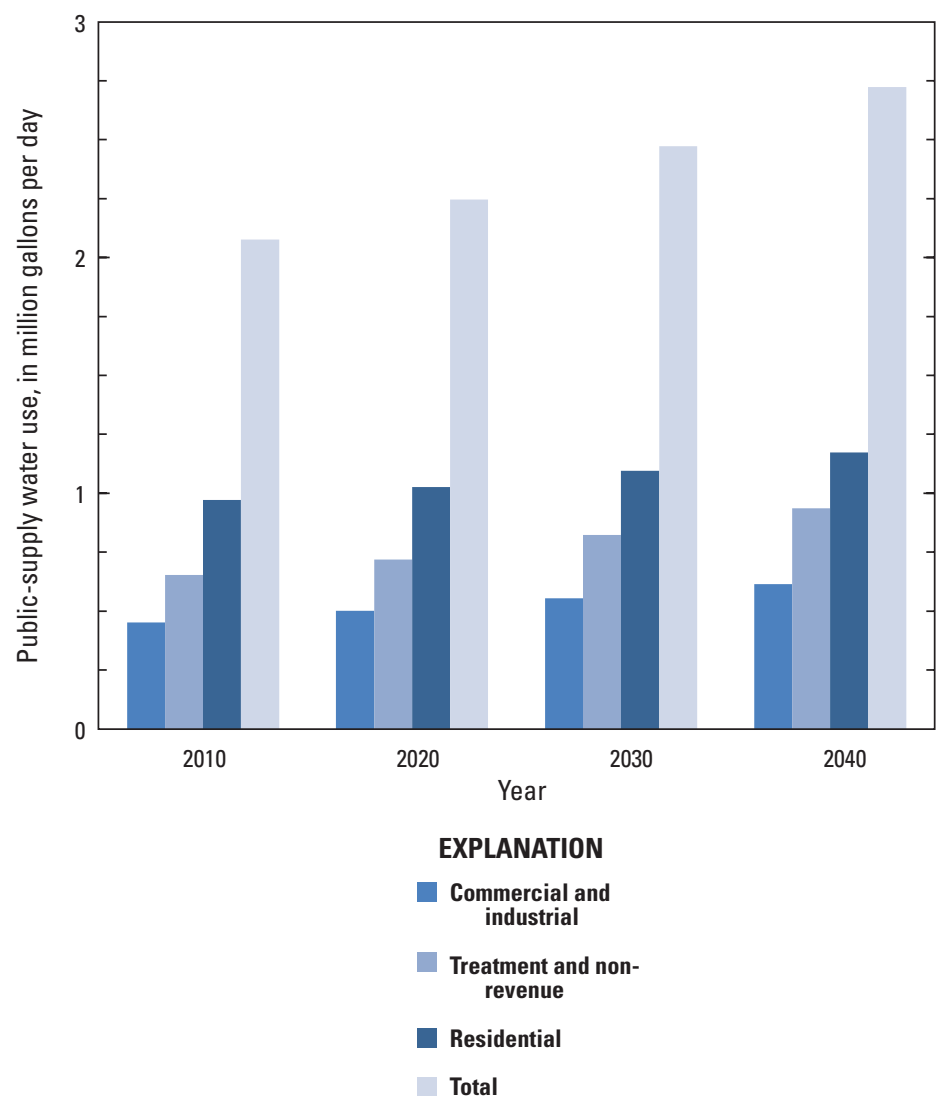

Figure 17. Public-supply water use in 2010 and projected public-supply water use for 2020-2040, Dale Hollow reservoir catchment area, Cumberland River watershed.

Table 13. Population served, public-supply water use during 2010, and public-supply water-use projections in the Dale Hollow reservoir catchment area, 2020, 2030, and 2040.

[Values in million gallons per day except where noted]

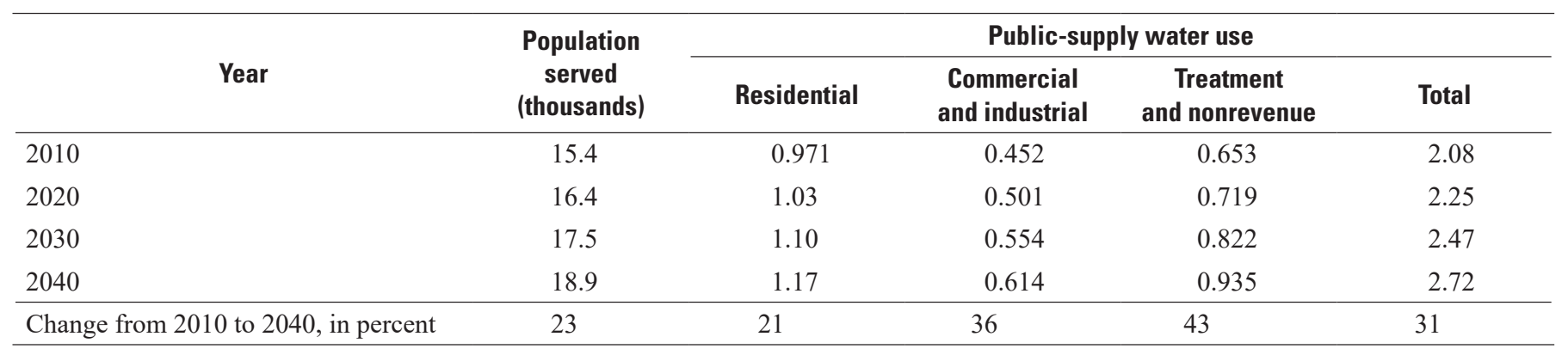




\section{Cordell Hull Reservoir Catchment Area}

Population and water withdrawals are expected to increase through 2040 in the Cordell Hull RCA. During 2010, the population served for each system in the Cordell Hull RCA ranged from about 300 to 10,000 people, and the total for all systems was about 36,000 people. Raw water withdrawals by six principal suppliers in the Cordell Hull RCA totaled 4.72 Mgal/d, ranging from 0.383 to $2.24 \mathrm{Mgal} / \mathrm{d}$. For nine systems in the Tennessee portion of the Cordell Hull RCA, residential use per capita ranged from 47 to 70 gallons per day. Nonrevenue water in the Tennessee portion of the Cordell Hull RCA totaled $2.00 \mathrm{Mgal} / \mathrm{d}$, which was 42 percent of the raw water withdrawals by principal suppliers. The ratios of commercial and industrial to residential water use were determined for six water-supply systems located in the Tennessee portion of the Cordell Hull RCA and ranged from 0.023 to 0.746 .

Because population served is projected to increase by about 2,600 people from 2010 to 2040, the supply of raw water and (or) finished water to meet internal demand in the Cordell Hull RCA is projected to increase by $0.391 \mathrm{Mgal} / \mathrm{d}$, or 8 percent (from 4.99 to $5.38 \mathrm{Mgal} / \mathrm{d}$; table 14; fig. 18). Livingston Water Department, which relies on the Cumberland River for supply, is projected to provide $0.312 \mathrm{Mgal} / \mathrm{d}$ of this projected increase. Celina Water System is projected to provide $0.033 \mathrm{Mgal} / \mathrm{d}$ of the increase and relies on the Obey River. Northwest Clay County Utility is projected to provide $0.021 \mathrm{Mgal} / \mathrm{d}$ of the increase and relies on the Cumberland River. From 2010 to 2040, the percentage of change in the supply of raw water and (or) finished water to meet internal demand for all systems in the Cordell Hull RCA is projected to range from -13 to 19 percent.
During 2010 to 2040, the increase in public-supply water use is projected to be $0.495 \mathrm{Mgal} / \mathrm{d}$, or 12 percent, in the Tennessee portion of the Cordell Hull RCA. Of this projected increase, 38 percent of the total increase $(0.186 \mathrm{Mgal} / \mathrm{d})$ is expected to be delivered as finished water to residential customers, 17 percent $(0.082 \mathrm{Mgal} / \mathrm{d})$ as finished water to commercial and industrial customers, and the remainder (46 percent) as nonrevenue water. In the Tennessee portion of the Cordell Hull RCA, commercial and industrial water use represents 13 percent of the total finished water from 2010 through 2040.

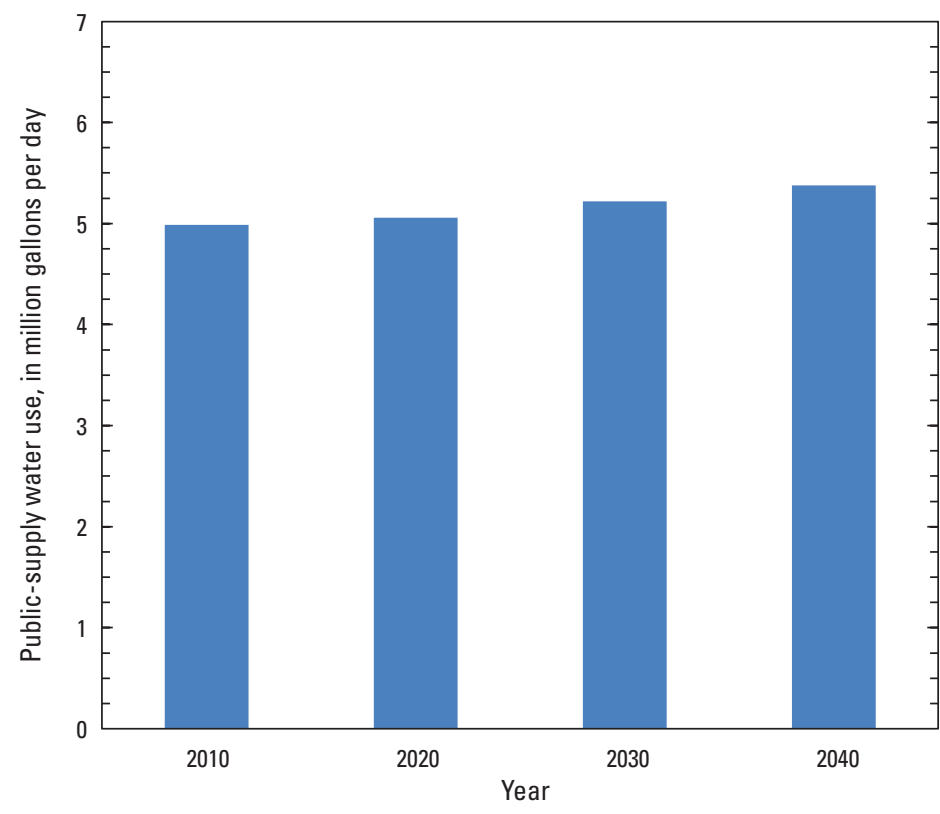

Figure 18. Public-supply water use in 2010 and projected public-supply water use for 2020-2040, Cordell Hull reservoir catchment area, Cumberland River watershed.

Table 14. Population served, public-supply water use during 2010, and public-supply water-use projections in the Cordell Hull reservoir catchment area, 2020, 2030, and 2040.

[Values in million gallons per day except where noted]

\begin{tabular}{|c|c|c|c|c|c|}
\hline \multirow[b]{2}{*}{ Year } & \multirow{2}{*}{$\begin{array}{c}\text { Population } \\
\text { served }{ }^{1} \\
\text { (thousands) }\end{array}$} & \multicolumn{4}{|c|}{ Public-supply water use } \\
\hline & & Residential' $^{2}$ & $\begin{array}{c}\text { Commercial } \\
\text { and industrial }\end{array}$ & $\begin{array}{c}\text { Treatment } \\
\text { and nonrevenue }\end{array}$ & Total $^{1}$ \\
\hline 2020 & 36.7 & 1.73 & 0.549 & 2.03 & 5.06 \\
\hline 2030 & 37.8 & 1.80 & 0.580 & 2.12 & 5.22 \\
\hline
\end{tabular}

${ }^{1}$ Values for the Tennessee and Kentucky portion of the Cordell Hull reservoir catchment area.

${ }^{2}$ Values for the Tennessee portion of the Cordell Hull reservoir catchment area. 


\section{Center Hill Reservoir Catchment Area}

Population and water withdrawals are expected to increase through 2040 in the Center Hill RCA. During 2010, the population served for each system in the Center Hill RCA ranged from about 100 to 32,000 people, and the total for all systems was about 142,000 people. Raw water withdrawals by nine principal suppliers in the Center Hill RCA ranged from 0.587 to $13.3 \mathrm{Mgal} / \mathrm{d}$. Residential use per capita ranged from 52 to 105 gallons per day. The ratios of commercial and industrial to residential water use were determined for 17 water-supply systems in the Center Hill RCA and ranged from 0.023 to 1.69 . Nonrevenue water in the Center Hill RCA totaled $10.3 \mathrm{Mgal} / \mathrm{d}$, which was 43 percent of the raw water withdrawals by principal suppliers.

Because population served is projected to increase by about 56,000 people from 2010 to 2040 , the supply of raw water and (or) finished water to meet internal demand in the Center Hill RCA is projected to increase by 42 percent (from 24.6 to $34.9 \mathrm{Mgal} / \mathrm{d}$; table 15 ; fig. 19). Of this projected increase, 35 percent $(3.56 \mathrm{Mgal} / \mathrm{d})$ is expected to be delivered as finished water to residential customers, 27 percent $(2.74 \mathrm{Mgal} / \mathrm{d})$ as finished water to commercial and industrial customers, and the remainder (39 percent, or $3.96 \mathrm{Mgal} / \mathrm{d}$ ) as nonrevenue water. Forty-seven percent of the increase is projected to come from the Cookeville Water Department, which relies on Caney Fork. All other systems in the Center Hill RCA are projected to contribute from less than 1 percent to 5 percent of the increase in water supply. Other sources of water in the Center Hill RCA include Barren Fork River, Bee Creek, Billy's Branch Lake, Calf Killer River, Collins River, Laurel Lake, and Ranger Creek Impoundment.

From 2010 to 2040, the percentage of increase in the supply of raw water and (or) finished water to meet internal demand for all systems in the Center Hill RCA — with the exception of Big Creek Utility District- is projected to range from 2 to 77 percent. During the same period, the projected percentage of decrease in the supply of raw water and (or) finished water to meet internal demand for Big Creek Utility District is 1 percent, or less than $0.008 \mathrm{Mgal} / \mathrm{d}$. In the Center Hill RCA, commercial and industrial water use represented 22 percent $(5.41 \mathrm{Mgal} / \mathrm{d})$ of the total finished water in 2010. By 2040, commercial and industrial water use is projected to be 23 percent $(8.15 \mathrm{Mgal} / \mathrm{d})$ of the total finished water, a 51-percent increase from 2010 (fig. 19). Residential water use is projected to increase 40 percent, from 8.93 to $12.5 \mathrm{Mgal} / \mathrm{d}$, from 2010 to 2040 and be 36 percent of the total finished water.

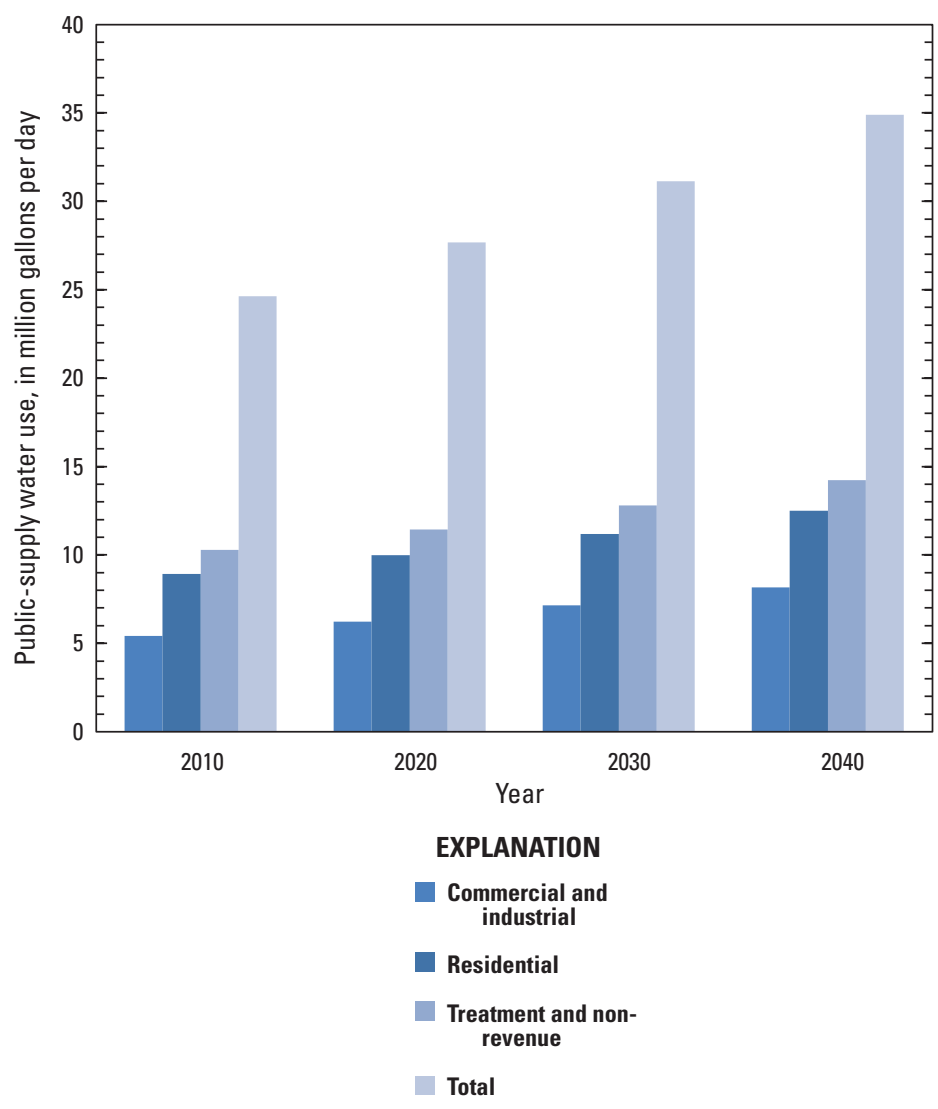

Figure 19. Public-supply water use in 2010 and projected public-supply water use for 2020-2040, Center Hill reservoir catchment area, Cumberland River watershed.

Table 15. Population served, public-supply water use during 2010, and public-supply water-use projections in the Center Hill reservoir catchment area, 2020, 2030, and 2040.

[Values in million gallons per day except where noted]

\begin{tabular}{lccccc}
\hline \multirow{2}{*}{ Year } & $\begin{array}{c}\text { Population } \\
\text { served }\end{array}$ & \multicolumn{4}{c}{ Public-supply water use } \\
\cline { 3 - 6 } & (thousands) & Residential & $\begin{array}{c}\text { Commercial } \\
\text { and industrial }\end{array}$ & $\begin{array}{c}\text { Treatment } \\
\text { and nonrevenue }\end{array}$ & Total \\
\hline 2010 & 142 & 8.93 & 5.41 & 10.3 & 24.6 \\
2020 & 158 & 10.0 & 6.23 & 11.4 & 27.7 \\
2030 & 177 & 11.2 & 7.14 & 12.8 & 31.1 \\
2040 & 198 & 12.5 & 8.15 & 14.2 & 34.9 \\
\hline Change from 2010 to 2040, in percent & 40 & 40 & 51 & 39 & 42 \\
\hline
\end{tabular}




\section{Old Hickory Reservoir Catchment Area}

Population and water withdrawals are expected to increase through 2040 in the Old Hickory RCA. During 2010, the population served for each system in the Old Hickory RCA ranged from about 140 to 73,000 people, and the total for all systems was about 284,000 people. Raw water withdrawals by 12 principal suppliers in the Old Hickory RCA totaled $43.3 \mathrm{Mgal} / \mathrm{d}$. Residential use per capita ranged from 48 to 73 gallons per day. The ratios of commercial and industrial to residential water use were determined for 14 water-supply systems in the Old Hickory RCA and ranged from 0.002 to 1.19. Nonrevenue water in the Old Hickory RCA totaled 17.7 Mgal/d, which was 41 percent of the raw water withdrawals by principal suppliers.

Because population served is projected to increase by about 133,000 people from 2010 to 2040 , the supply of raw water and (or) finished water to meet internal demand in the Old Hickory RCA is projected to increase by 59 percent (from 41.9 to $66.7 \mathrm{Mgal} / \mathrm{d}$; table 16; fig. 20). Of this projected increase, 35 percent $(8.69 \mathrm{Mgal} / \mathrm{d})$ is expected to be delivered as finished water to residential customers, 25 percent (5.67 Mgal/d) as finished water to commercial and industrial customers, and the remainder (42 percent, or $10.5 \mathrm{Mgal} / \mathrm{d}$ ) as nonrevenue water. Thirty-seven percent of this increased water demand is projected to come from White House Utility District, which relies on the Cumberland River for supply. West Wilson Utility District is projected to provide 19 percent of the increase and relies on the Cumberland River. Thirteen percent of this water is projected to come from Hendersonville Utility District, which also relies on the Cumberland River. Gallatin Water Department is projected to provide 11 percent of the increase and relies on the Cumberland River. Other sources of water in the Old Hickory RCA include Caney Fork and five wells that supply more than 2 percent of the total. From 2010 to 2040 , the percentage of increase in the supply of raw water and (or) finished water to meet internal demand for all systems in the Old Hickory RCA is projected to range from
22 to 81 percent. Across the Old Hickory RCA, commercial and industrial water use represented 14 percent $(5.73 \mathrm{Mgal} / \mathrm{d})$ of the total finished water in 2010. By 2040, commercial and industrial water use is projected to be 17 percent $(11.4 \mathrm{Mgal} / \mathrm{d})$ of the total finished water, a 99-percent increase from 2010 (table 16; fig. 20). Residential water use is projected to increase 47 percent, from 18.4 to $27.1 \mathrm{Mgal} / \mathrm{d}$, from 2010 to 2040 .

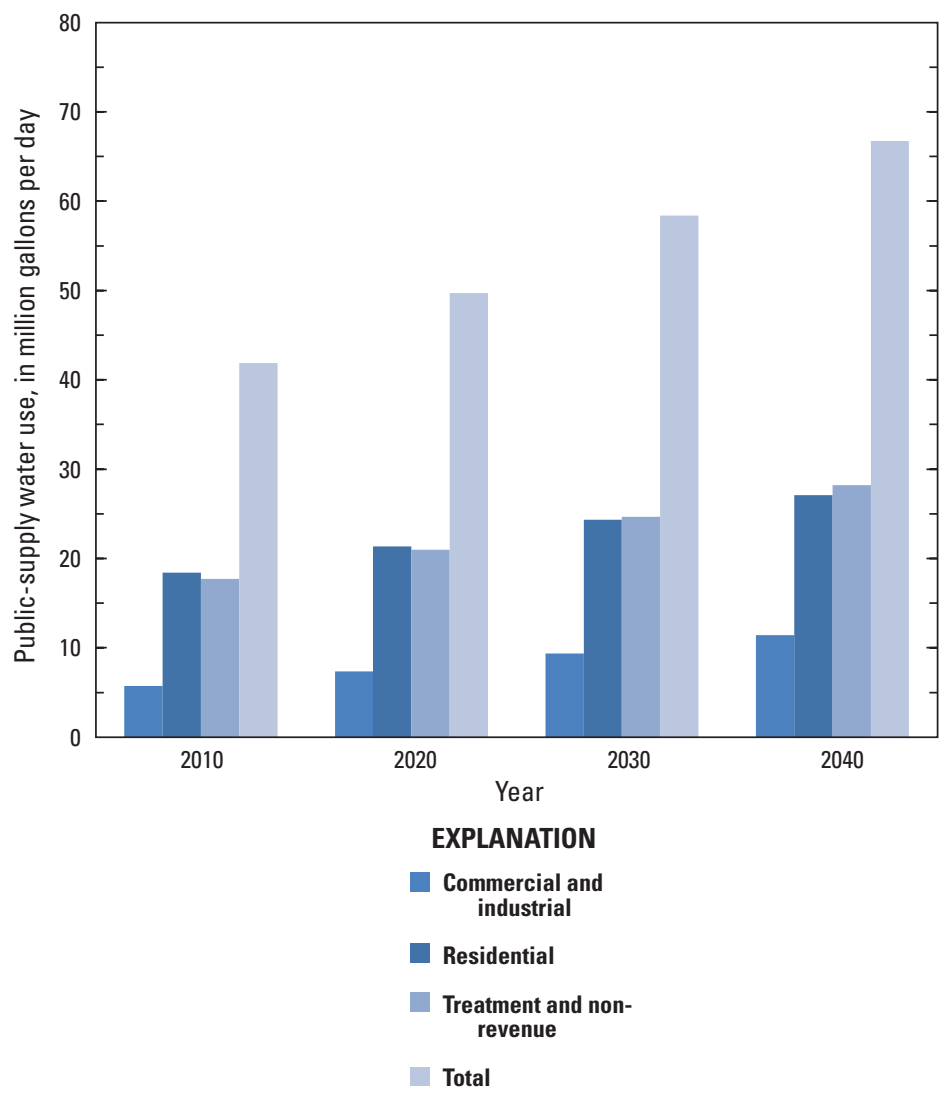

Figure 20. Public-supply water use in 2010 and projected public-supply water use for 2020-2040, Old Hickory reservoir catchment area, Cumberland River watershed.

Table 16. Population served, public-supply water use during 2010, and public-supply water-use projections in the Old Hickory reservoir catchment area, 2020, 2030, and 2040.

[Values in million gallons per day except where noted]

\begin{tabular}{|c|c|c|c|c|c|}
\hline \multirow[b]{2}{*}{ Year } & \multirow{2}{*}{$\begin{array}{c}\text { Population } \\
\text { served } \\
\text { (thousands) }\end{array}$} & \multicolumn{4}{|c|}{ Public-supply water use } \\
\hline & & Residential & $\begin{array}{l}\text { Commercial } \\
\text { and industrial }\end{array}$ & $\begin{array}{c}\text { Treatment } \\
\text { and nonrevenue }\end{array}$ & Total \\
\hline 2020 & 329 & 21.4 & 7.36 & 21.0 & 49.7 \\
\hline 2030 & 375 & 24.3 & 9.35 & 24.7 & 58.4 \\
\hline
\end{tabular}




\section{J. Percy Priest Reservoir Catchment Area}

Population and water withdrawals are expected to increase through 2040 in the J. Percy Priest RCA. During 2010, the population served for each system in the J. Percy Priest RCA ranged from about 9,600 to 98,000 people, and the total for all systems was about 256,000 people. Raw water withdrawals by six principal suppliers in the J. Percy Priest RCA ranged from 1.14 to $9.95 \mathrm{Mgal} / \mathrm{d}$. Residential use per capita ranged from 56 to 66 gallons per day. The ratios of commercial and industrial to residential water use were determined for all water-supply systems in the J. Percy Priest RCA and ranged from 0.086 to 1.37 . Nonrevenue water in the J. Percy Priest RCA totaled 7.5 Mgal/d, which is 24 percent of the raw water withdrawals by principal suppliers.

Because population served is projected to increase to about 520,000 people from 2010 to 2040, the supply of raw water and (or) finished water to meet internal demand in the J. Percy Priest RCA is projected to increase by 130 percent (from 34.4 to $78.9 \mathrm{Mgal} / \mathrm{d}$; table 17; fig. 21). Of this projected increase, 36 percent $(15.9 \mathrm{Mgal} / \mathrm{d})$ is projected to be delivered as finished water to residential customers, 42 percent $(18.9 \mathrm{Mgal} / \mathrm{d})$ is projected to be delivered as finished water to commercial and industrial customers, and the remainder, 22 percent $(9.77 \mathrm{Mgal} / \mathrm{d})$, is projected to be nonrevenue water. Thirty-seven percent of this increased supply is projected to come from Consolidated Utility District of Rutherford County, which relies on the East Fork Stones River for supply. Murfreesboro Water Department is projected to provide 25 percent of the increase and relies on two water sources: the Stones River Pump Station and East Fork Stones River. Twenty-one percent of this water is projected to come from LaVergne Water System, which relies on Percy Priest Lake and Smyrna Water System for supply. Thirteen percent of this water is projected to come from Smyrna Water System, which relies on Percy Priest Lake for supply. Other sources of water in the J. Percy Priest RCA include Gladeville Utility District \#1, which relies on two wells, and Woodbury Water System, which relies on the East Fork Stones River. The combined total from Gladeville Utility District \#1 and Woodbury Water System is less than 4 percent of the projected increase. From 2010 to 2040, the percentage of increase in the supply of raw water and (or) finished water to meet internal demand for all systems in the J. Percy Priest RCA is projected to range from 19 to 237 percent. In the J. Percy Priest RCA, commercial and industrial water use represented 29 percent $(10.0 \mathrm{Mgal} / \mathrm{d})$ of the total finished water in 2010. By 2040, commercial and industrial water use is projected to be 37 percent $(28.9 \mathrm{Mgal} / \mathrm{d})$ of the total finished water-a 188-percent increase from 2010 (table 17; fig. 21). Residential water use is expected to increase by 95 percent, from 16.8 to 32.7 Mgal/d, from 2010 to 2040 .

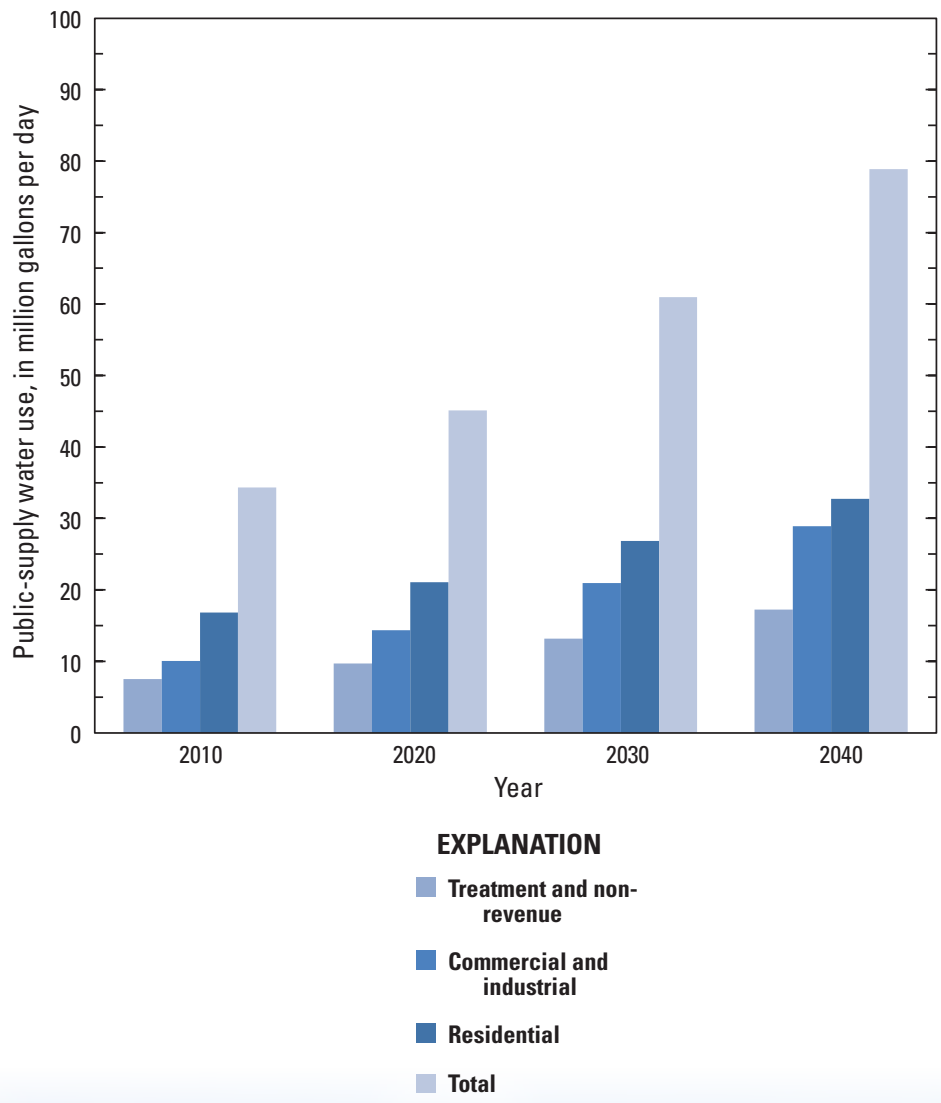

Figure 21. Public-supply water use in 2010 and projected public-supply water use for 2020-2040, J. Percy Priest reservoir catchment area, Cumberland River watershed.

Table 17. Population served, public-supply water use during 2010, and public-supply water-use projections in the J. Percy Priest reservoir catchment area, 2020, 2030, and 2040.

[Values in million gallons per day except where noted]

\begin{tabular}{lcccccc}
\hline & Year & $\begin{array}{c}\text { Population } \\
\text { served }\end{array}$ & \multicolumn{4}{c}{ Public-supply water use } \\
\cline { 5 - 7 } & (thousands) & Residential & $\begin{array}{c}\text { Commercial } \\
\text { and industrial }\end{array}$ & $\begin{array}{c}\text { Treatment } \\
\text { and nonrevenue }\end{array}$ & Total \\
\hline 2010 & 256 & 16.8 & 10.0 & 7.5 & 34.4 \\
2020 & 335 & 21.1 & 14.3 & 9.7 & 45.1 \\
2030 & 426 & 26.8 & 20.9 & 13.2 & 61.0 \\
2040 & 520 & 32.7 & 28.9 & 17.3 & 78.9 \\
\hline Change from 2010 to 2040, in percent & 103 & 95 & 188 & 131 & 130 \\
\hline
\end{tabular}




\section{Cheatham Reservoir Catchment Area}

Population and water withdrawals are expected to increase through 2040 in the Cheatham RCA. During 2010, the population served for each system in the Cheatham RCA ranged from about 1,200 to 379,000 people, and the total for all systems was about 689,000 people. Raw water withdrawals by 10 principal suppliers in the Cheatham RCA ranged from 0.112 to $102.34 \mathrm{Mgal} / \mathrm{d}$. Residential use per capita ranged from 48 to 129 gallons per day. The ratios of commercial and industrial to residential water use were determined for 14 water-supply systems in the Cheatham RCA and ranged from 0.014 to 1.40 . Nonrevenue water in the Cheatham RCA totaled $58.4 \mathrm{Mgal} / \mathrm{d}$, which is 40 percent of the raw water withdrawals by principal suppliers.

Because population served is projected to increase by about 260,000 people from 2010 to 2040, the supply of raw water and (or) finished water to meet internal demand in the Cheatham RCA is projected to increase by 45 percent (from 148 to $214 \mathrm{Mgal} / \mathrm{d}$; table 18; fig. 22). Of this projected increase, more than 31 percent $(20.2 \mathrm{Mgal} / \mathrm{d})$ is expected to be delivered as finished water to residential customers, 29 percent (19.1 Mgal/d) is expected to be delivered as finished water to commercial and industrial customers, and the remainder (40 percent, or $26.6 \mathrm{Mgal} / \mathrm{d}$ ) is expected to be nonrevenue water. Thirty-eight percent of this increased demand will come from Nashville Water Department \#1, which relies on the Cumberland River for supply. Franklin Water Department is projected to provide 16 percent of the increase and relies on the Harpeth River and Harpeth Valley Utility District. Other sources of water in the Cheatham RCA include Big Marrow Bone Lake, Sycamore Creek, Turnbull Creek, and three wells that are projected to supply less than 9 percent of the total increase. From 2010 to 2040, the percentage of increase in the supply of raw water and (or) finished water to meet internal demand for all systems in the Cheatham RCA ranges from 8 to 170 percent. In the Cheatham RCA, commercial and industrial water use represented 28 percent $(40.8 \mathrm{Mgal} / \mathrm{d})$ of the total finished water in 2010. By 2040, commercial and industrial water use is projected to be $59.9 \mathrm{Mgal} / \mathrm{d}$ (28 percent of the total finished water), which is a 47-percent increase from 2010 (table 18; fig. 22). Residential water use is projected to increase by 41 percent, from 48.9 to $69.1 \mathrm{Mgal} / \mathrm{d}$, from 2010 to 2040 .

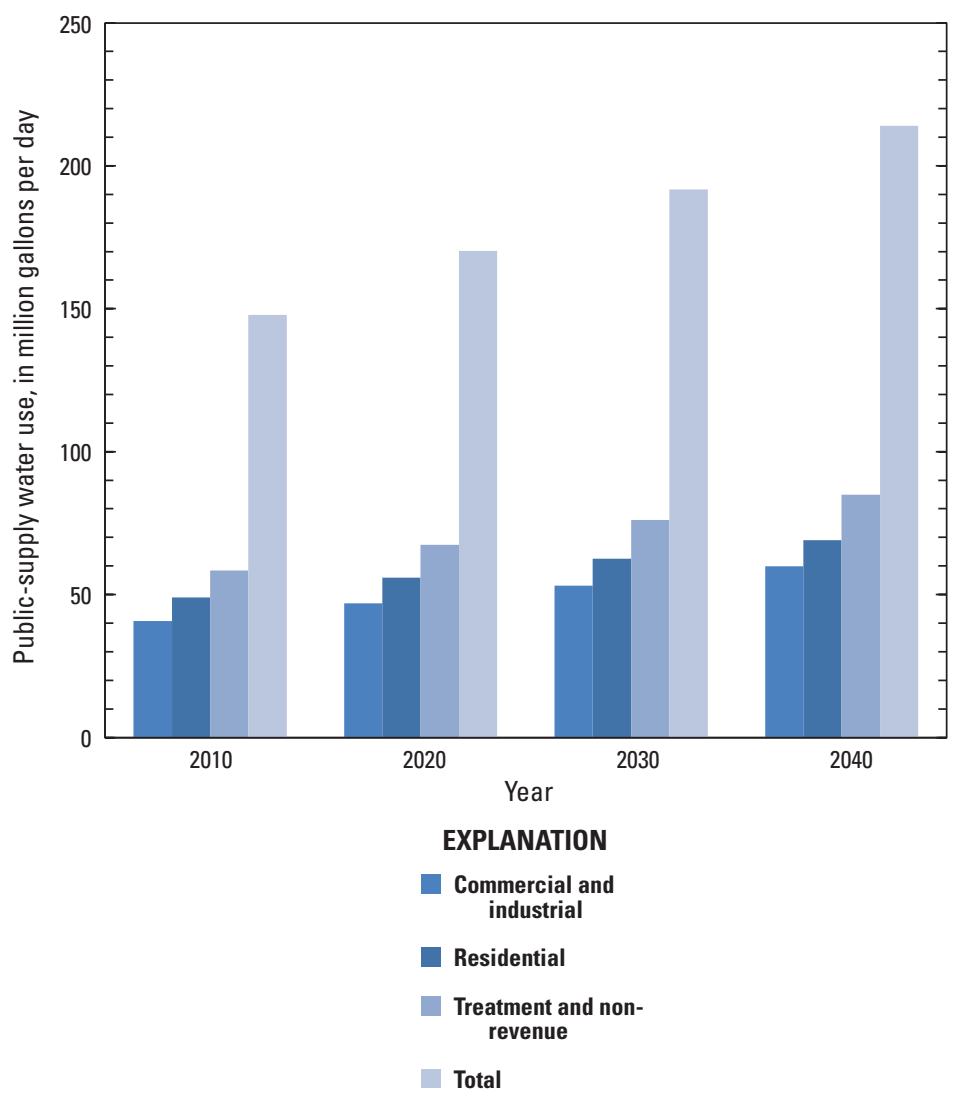

Figure 22. Public-supply water use in 2010 and projected public-supply water use for 2020-2040, Cheatham reservoir catchment area, Cumberland River watershed.

Table 18. Population served, public-supply water use during 2010, and public-supply water-use projections in the Cheatham reservoir catchment area, 2020, 2030, and 2040.

[Values in million gallons per day except where noted]

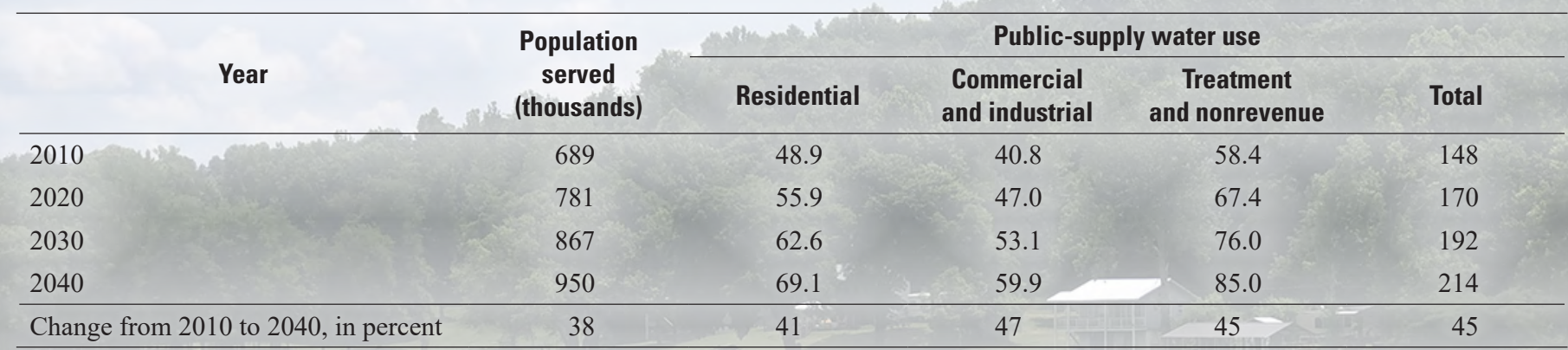




\section{Barkley Reservoir Catchment Area}

Population and water withdrawals are expected to increase through 2040 in the Barkley RCA. During 2010, the population served for each system in the Barkley RCA ranged from about 25 to 131,000 people, and the total for all systems was about 331,000 people. Raw water withdrawals by 20 principal suppliers in the Barkley RCA totaled $51.3 \mathrm{Mgal} / \mathrm{d}$, ranging from 0.002 to $16.0 \mathrm{Mgal} / \mathrm{d}$. For 18 systems in the Tennessee portion of the Barkley RCA, residential use per capita ranged from 47 to 68 gallons per day. Nonrevenue water in the Tennessee portion of the Barkley RCA totaled $11.9 \mathrm{Mgal} / \mathrm{d}$, which is 23 percent of the raw water withdrawals by principal suppliers. The ratios of commercial and industrial to residential water use were determined for 14 water-supply systems located in the Tennessee portion of the Barkley RCA and ranged from 0.001 to 5.10 .

Because population served is projected to increase by about 130,000 people from 2010 to 2040, the supply of raw water and (or) finished water to meet internal demand in the Barkley RCA is projected to increase by $21.0 \mathrm{Mgal} / \mathrm{d}$, or 41 percent (from 51.5 to $72.5 \mathrm{Mgal} / \mathrm{d}$; table 19; fig. 23). Clarksville Water Department, which relies on the Cumberland River for supply, is projected to provide $9.68 \mathrm{Mgal} / \mathrm{d}$ of the projected increase. Hopkinsville Water Environmental Authority is projected to provide $4.34 \mathrm{Mgal} / \mathrm{d}$ of the increase and relies on Lake Barkley, North Fork Little River, North Quarry, and South Quarry. Springfield Water System, which relies on the Red River, is projected to provide $1.82 \mathrm{Mgal} / \mathrm{d}$ of the increase. Fort Campbell Water System is projected to provide $0.957 \mathrm{Mgal} / \mathrm{d}$ of the increase and relies on Boiling Spring. Other sources of water in the Barkley RCA include Cadiz Spring, Columbia Caverns Spring, Yellow Creek, and four wells that are projected to supply $0.253 \mathrm{Mgal} / \mathrm{d}$ of the increase. Eddyville Water Department (Lake Barkley and two wells), Kentucky State Penitentiary (Kentucky Lake), Kuttuwa Water Department (Lake Barkley), and Princeton Water \& Sewer Commission (Lake Barkley) are expected to have a combined decrease in supply of $0.069 \mathrm{Mgal} / \mathrm{d}$. From 2010 to 2040 , the percentage of change in the supply of raw water and (or) finished water to meet internal demand for all systems in the Barkley RCA is expected to range from -2 to 201 percent.

During 2010 to 2040, the projected increase in publicsupply water use is $15.7 \mathrm{Mgal} / \mathrm{d}$, or 53 percent, in the Tennessee portion of the Barkley RCA. Of this projected increase, 35 percent of the increase $(5.55 \mathrm{Mgal} / \mathrm{d})$ is expected to be delivered as finished water to residential customers, 28 percent $(4.45 \mathrm{Mgal} / \mathrm{d})$ is expected to be delivered as finished water to commercial and industrial customers, and the remainder (36 percent) is expected to be nonrevenue water. In the Tennessee portion of the Barkley RCA, commercial and industrial water use represented 20 percent $(5.91 \mathrm{Mgal} / \mathrm{d})$ of the total finished water in 2010. By 2040, commercial and industrial water use is projected to be $10.4 \mathrm{Mgal} / \mathrm{d}$ (23 percent of the total finished water), which is a 75-percent increase from 2010 (table 19).

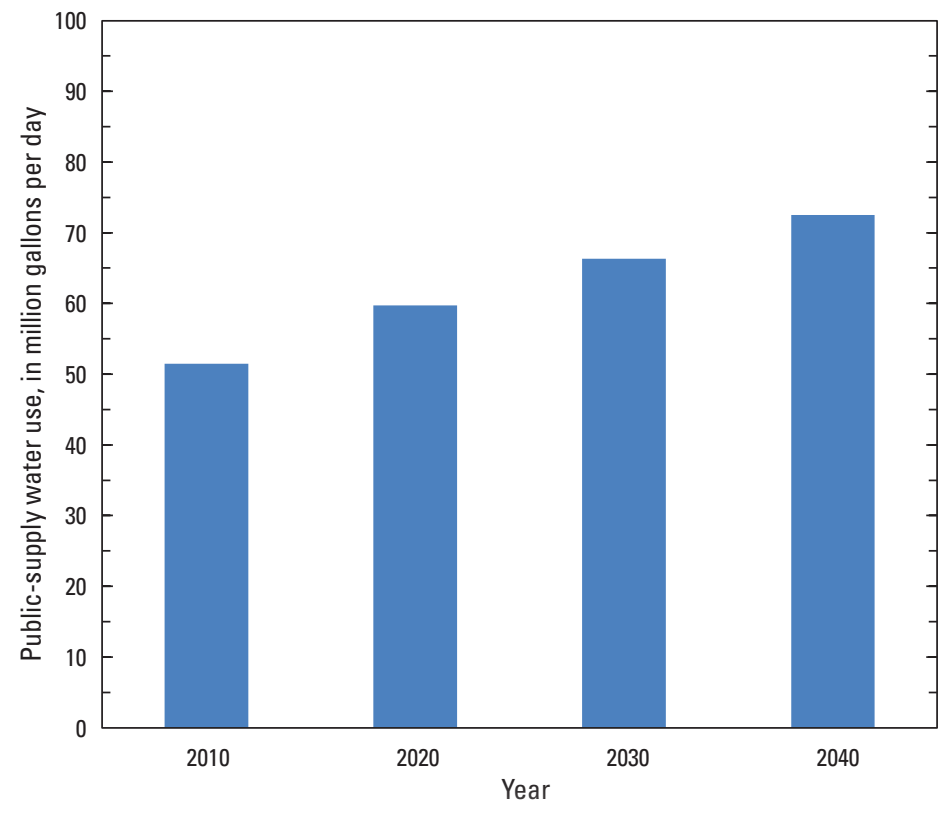

Figure 23. Public-supply water use in 2010 and projected public-supply water use for 2020-2040, Barkley reservoir catchment area, Cumberland River watershed.

Table 19. Population served, public-supply water use during 2010, and public-supply water-use projections in the Barkley reservoir catchment area, 2020, 2030, and 2040.

[Values in million gallons per day except where noted]

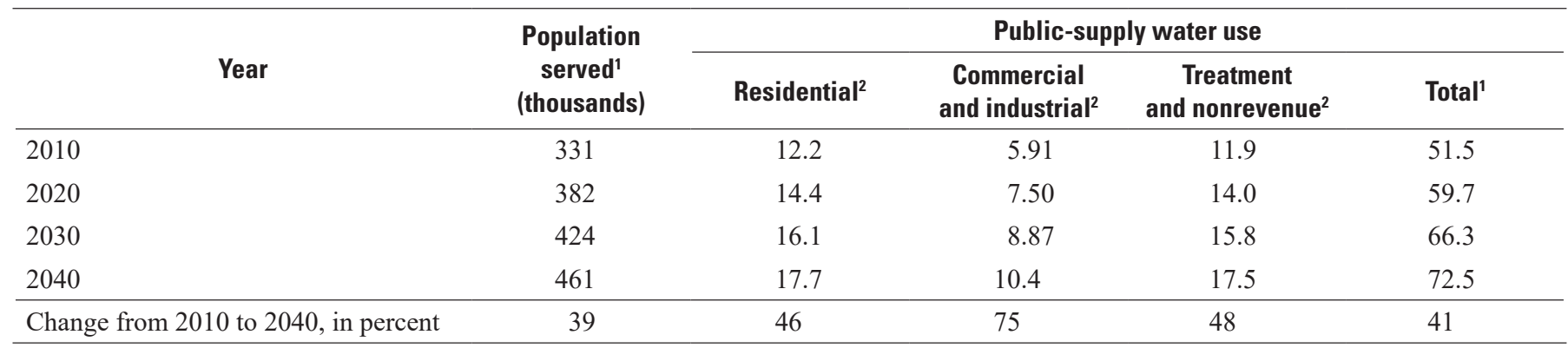

${ }^{1}$ Values for the Tennessee and Kentucky portion of the Barkley reservoir catchment area.

${ }^{2}$ Values for the Tennessee portion of the Barkley reservoir catchment area. 


\section{Summary}

As part of ongoing water-supply analyses of USACE reservoirs in the Cumberland River watershed, the USACE is identifying areas where potential water-resources issues may arise in the future. The USGS, in cooperation with the USACE, collected and analyzed water-use data for 10 RCAs to provide estimates of existing (2010) and future (2020, 2030, and 2040) water use in the Cumberland River watershed.

The water-use categories evaluated include thermoelectric power, industrial, public supply, and irrigation. During 2010, withdrawals were estimated as follows: thermoelectric power, 3,051.12 million gallons per day (Mgal/d); public supply, $360.00 \mathrm{Mgal} / \mathrm{d}$; industrial, $31.5 \mathrm{Mgal} / \mathrm{d}$; and irrigation, $13.6 \mathrm{Mgal} / \mathrm{d}$. The estimated return flow for thermoelectric water use in 2010 was $3,051.06 \mathrm{Mgal} / \mathrm{d}$, and return flow for industrial and public supply combined was an estimated $319.02 \mathrm{Mgal} / \mathrm{d}$. For thermoelectric power, consumptive use was estimated as $0.06 \mathrm{Mgal} / \mathrm{d}$. Industrial and publicsupply consumptive use totaled $72.5 \mathrm{Mgal} / \mathrm{d}$, and irrigation consumptive use was $13.6 \mathrm{Mgal} / \mathrm{d}$.

Estimates of water withdrawals by source indicate that during 2010, total surface-water withdrawals comprised more than 99 percent of the total withdrawals, or 3,437.90 Mgal/d. Total groundwater withdrawals were $18.33 \mathrm{Mgal} / \mathrm{d}$, or less than 1 percent of the total. Thermoelectric power withdrawals were large and exclusively from surface water in the watershed. In 2010, most of the estimated $18.33 \mathrm{Mgal} / \mathrm{d}$ of groundwater was used for irrigation (9.95 Mgal/d).

From 2010 to 2040, the supply of raw and (or) finished water to meet demand for all 10 RCAs in the Cumberland River watershed is projected to increase from 360.10 to $532.51 \mathrm{Mgal} / \mathrm{d}$, or 48 percent. These projected increases include a 41-percent increase, from 51.5 to $72.5 \mathrm{Mgal} / \mathrm{d}$, in the Barkley RCA. The Barkley RCA provides water to Clarksville, Tennessee, which is the second largest population center in the study area. From 2010 to 2040, the combined total water demand for the Cheatham, J. Percy Priest, and Old Hickory RCAs is projected to increase from 224.08 to $359.58 \mathrm{Mgal} / \mathrm{d}$, or 61 percent. The Cheatham RCA provides water to the city of Nashville, Tennessee, the largest population center in the study area. The J. Percy Priest RCA provides water to the city of Murfreesboro, Tennessee, the third largest population center in the study area. From 2010 to 2040, the combined total water demand for the Center Hill, Cordell Hull, and Dale Hollow RCAs is projected to increase from 31.7 to $43.0 \mathrm{Mgal} / \mathrm{d}$, or 36 percent. From 2010 to 2040, the combined total water demand for the Martins Fork, Laurel, and Wolf Creek RCAs is projected to increase from 52.8 to $57.4 \mathrm{Mgal} / \mathrm{d}$, or 9 percent. Martins Fork is the only RCA in the Cumberland River watershed with a projected decrease in water use from 2010 to 2040.

\section{References}

Case, P.J., and Alward, G.S., 1997, Patterns of demographic, economic, and value change in the western United StatesImplications for water use and management: Report to the Western Water Policy Review Advisory Commission, 35 p.

Center for Business and Economic Research, The University of Tennessee, 2012, Tennessee county population projections, accessed January 2013 at http://cber.bus.utk.edu/data/ cntypjoct12.xls.

Diehl, T.H., and Harris, M.A., 2014, Withdrawal and consumption of water by thermoelectric power plants in the United States, 2010: U.S. Geological Survey Scientific Investigation Report 2014-5184, 28 p., accessed May 2016 at http://dx.doi.org/10.3133/sir20145184.

Hutson, S.S., Koroa, M.C., and Murphee, C.M., 2003, Estimated use of water in the Tennessee River watershed in 2000 and projections of water use to 2030: U.S. Geological Survey Water-Resources Investigations Report 2003-4302, 89 p.

Kenny, J.F., Barber, N.L., Hutson, S.S., Linsey, K.S., Lovelace, J.K., and Maupin, M.A., 2009, Estimated use of water in the United States in 2005: U.S. Geological Survey Circular 1344, 52 p.

Kentucky State Data Center [KSDC], 2012, Population projections 2015-2050, accessed January 2012 at http:// ksdc.louisville.edu/index.php/kentucky-demographic-data/ projections.

Lins, H.F., Hirsch, R.M., and Kiang, J.K., 2010, Water-The nation's fundamental climate issue-A white paper on the U.S. Geological Survey role and capabilities: U.S. Geological Survey Circular 1347, 9 p. [Also available at http://pubs. usgs.gov/circ/1347/.]

Maupin, M.A., Kenny, J.F., Hutson, S.S., Lovelace, J.K., Barber, N.L., and Linsey, K.S., 2014, Estimated use of water in the United States in 2010: U.S. Geological Survey Circular 1405, 56 p., accessed December 2014 at http:// dx.doi.org/10.3133/cir1405.

National Oceanic and Atmospheric Administration, 2010, May $1 \& 22010$ epic flood event for western and Middle Tennessee: National Weather Service Weather Forecast Office, Nashville, Tennessee, accessed July 2014 at http://www.srh. noaa.gov/ohx/?n=may2010epicfloodevent.

National Oceanic and Atmospheric Administration, 2011, Extreme weather 2011-Mississippi River flooding, Spring-Summer, 2011, accessed March 2013 at http://www. noaa.gov/extreme2011/mississippi_flood.html. 
National Oceanic and Atmospheric Administration, 2013, Climate at a glance, accessed March 2013 at http://www.ncdc. noaa.gov/temp-and-precip/time-series/.

Robinson, J.A., 2017, Public supply water use in the Cumberland River watershed in 2010 and projections of public-supply water use to 2040: U.S. Geological Survey data release, https://doi.org/10.5066/F7M043KK.

Robinson, J.A., and Brooks, J.M., 2010, Public water-supply systems and associated water use in Tennessee, 2005: U.S. Geological Survey Open-File Report 2010-1226, 100 p.

Tennessee Department of Environment and Conservation, Division of Water Supply, 2011, unpublished data on file in the U.S. Geological Survey Lower Mississippi-Gulf Water Science Center office (Nashville, Tennessee), updated by the U.S. Geological Survey in 2013.

U.S. Census Bureau, 2010a, TIGER Products, accessed October 2012 at http://www.census.gov/geo/www/tiger.

U.S. Census Bureau, 2010b, Race and Hispanic or Latino: 2010 - State-Place, 2010 Census redistricting data (Public Law 94-171) summary file, accessed March 2013 at http://factfinder2.census.gov/faces/tableservices/jsf/ pages/productview.xhtml?pid=DEC_10_PL_GCTPL1. ST13\&prodType $=$ table $\#$.

U.S. Census Bureau, 2011, U.S. population projections, accessed January 2011 at http://www.census.gov/ population/www/projections/cohortcomponentmethod.html.

U.S. Census Bureau, 2012a, Kentucky-2010 Census of population and housing, population and housing unit counts: U.S Census Bureau, Report CPH-2-19, 105 p., accessed August 11, 2014, at http://www.census.gov/prod/cen2010/ cph-2-19.pdf.

U.S. Census Bureau, 2012b, Tennessee-2010 Census of population and housing, population and housing unit counts: U.S Census Bureau, Report CPH-2-44, 126 p., accessed August 11, 2014, at http://www.census.gov/prod/cen2010/ cph-2-44.pdf.

U.S. Department of Agriculture [USDA], 2009a, 2007 Census of agriculture, Kentucky State and county data, table 10, irrigation, 2007 and 2002: U.S. Department of Agriculture, 859 p., accessed March 14, 2013, at http://www.agcensus. usda.gov/Publications/2007/Full_Report/Volume_1, Chapter_1_State_Level/Kentucky/.

U.S. Department of Agriculture [USDA], 2009b, 2007 Census of agriculture, Tennessee State and county data, table 10, irrigation, 2007 and 2002: U.S. Department of Agriculture, 747 p., accessed March 14, 2013, at http://www.agcensus. usda.gov/Publications/2007/Full_Report/Volume_1, Chapter_2_County_Level/Tennessee/.
U.S. Department of Agriculture [USDA], 2010, 2007 Census of agriculture-Farm and ranch irrigation survey (2008): U.S. Department of Agriculture, National Agricultural Statistics Service, 268 p., accessed March 14, 2013, at http:// www.agcensus.usda.gov/Publications/Irrigation_Survey/.

U.S. Department of Agriculture [USDA], 2013, U.S. Department of Agriculture - Census of agriculture, accessed March 14, 2013, at http://www.agcensus.usda.gov/.

U.S. Department of Energy, Energy Information Administration [DOE, EIA], 2010a, Monthly Power Plant Report, EIA-923, accessed February 5, 2013, at http://www.eia.gov/ electricity/data/eia923/index.html.

U.S. Department of Energy, Energy Information Administration [DOE, EIA], 2010b, Monthly Power Plant Report, EIA-860, accessed February 5, 2013, at http://www.eia.gov/ electricity/data/eia860/index.html.

U.S. Drought Monitor, 2007, 2008, and 2012, U.S. Drought Monitor Archive, accessed July 2014 at http://droughtmonitor.unl.edu/MapsAndData/MapArchive.aspx.

U.S. Geological Survey [USGS], 2005 and 2010, Water use in the United States, accessed April 12, 2013, and July 16, 2013, at http://water.usgs.gov/watuse.

U.S. Geological Survey [USGS], 2007, Facing tomorrow's challenges-U.S. Geological Survey science in the decade 2007-2017: U.S. Geological Survey Circular 1309, $\mathrm{x}+70 \mathrm{p}$. 


\section{Glossary}

The following terms are referenced in the text or are part of the water-use Circular series.

commercial water use Water for motels, hotels, restaurants, office buildings, other commercial facilities, military and nonmilitary institutions, and (for 1990 and 1995) offstream fish hatcheries. Water may be obtained from a public-supply system or may be self-supplied.

consumptive use The part of water withdrawn that is evaporated, transpired, incorporated into products or crops, consumed by humans or livestock, or otherwise removed from the immediate water environment.

cooling system An equipment system that provides water for cooling purposes, such as to condensers at powerplants or at factories. May include water intakes, outlets, cooling towers, ponds, canals, pumps, and pipes.

domestic water use Water used for indoor household purposes, such as drinking, food preparation, bathing, washing clothes and dishes, and flushing toilets, and outdoor purposes, such as watering lawns and gardens. Domestic water use includes water provided to households by a public water supply (domestic deliveries from public suppliers) and selfsupplied water.

freshwater Water that contains less than1,000 milligrams per liter $(\mathrm{mg} / \mathrm{L})$ of dissolved solids. Generally, water with more than $500 \mathrm{mg} / \mathrm{L}$ of dissolved solids is undesirable for drinking and many industrial uses.

industrial water use Water used for fabrication, processing, washing, and cooling. Includes industries such as chemical and allied products, food, mining, paper and allied products, petroleum refining, and steel.

instream use Water that is used, but not withdrawn, from a surface-water source for such purposes as hydroelectricpower generation, navigation, water-quality improvement, fish propagation, and recreation.

irrigation water use Water that is applied by an irrigation system to assist crop and pasture growth, or to maintain vegetation on recreational lands such as parks and golf courses. Irrigation includes water that is applied for preirrigation, frost protection, chemical application, weed control, field preparation, crop cooling, harvesting, dust suppression, leaching of salts from the root zone, and conveyance losses.

offstream use Water withdrawn or diverted from a groundwater or surface-water source for commercial, selfsupplied, industrial, irrigation, public-supply, thermoelectric power, and other uses.

public-supply deliveries Amount of water delivered from a public supplier to users for domestic, commercial, industrial, thermoelectric power, or public-use purposes. public-supply water use Water withdrawn by public and private water suppliers that furnish water to at least 25 people or have a minimum of 15 connections. Public suppliers provide water for a variety of uses, such as domestic, commercial, industrial, thermoelectric-power, and public water use.

reclaimed wastewater Wastewater-treatment plant effluent that has been diverted for beneficial uses such as irrigation, industry, or thermoelectric-power cooling instead of being released to a natural waterway or aquifer.

reservoir catchment area The boundaries of a reservoir catchment area are determined by the natural drainage area truncated by a dam to account for water availability and the water-use transactions that occur within that drainage area. For this report, the reservoir catchment area accounts for the complete site-specific water-use transactions between adjoining reservoir catchment areas and is used to determine consumptive use at a large scale.

return flow Water that reaches a groundwater or surfacewater source after release from the point of use and thus becomes available for further use.

self-supplied water use Water withdrawn from a groundwater or surface-water source by a user rather than being obtained from a public-supply source.

thermoelectric-power water use Water used in the process of generating electricity with steam-driven turbine generators.

wastewater Water that carries wastes from homes, businesses, and industries.

wastewater treatment The processing of wastewater for the removal or reduction of contained solids or other undesirable constituents.

water-resources region Designated natural drainage basin or hydrologic area that contains either the drainage area of a major river or the combined drainage areas of 2 or more rivers; of 21 designated regions, 18 are in the conterminous United States, and 1 each is in Alaska, Hawaii, and the Caribbean.

water use In a restrictive sense, the term refers to water that is withdrawn for a specific purpose, such as for public supply, domestic use, irrigation, thermoelectric-power cooling, or industrial processing. More broadly, water use pertains to the interaction of humans with and influence on the hydrologic cycle and includes elements such as water withdrawal, delivery, consumptive use, wastewater release, reclaimed wastewater, return flow, and instream use.

water withdrawal Water removed from a groundwater or surface-water source for use.

watt-hour (Wh) An electrical energy unit of measure equal to 1 watt of power supplied to, or taken from, an electric circuit steadily for 1 hour. 
For more information about this publication, contact

Director, Lower Mississippi-Gulf Water Science Center U.S. Geological Survey

640 Grassmere Park, Suite 100

Nashville, Tennessee 37211

For additional information, visit https://www.usgs.gov/centers/lmg-water/

Publishing support provided by Lafayette Publishing Service Center 


\section{$\frac{\mathbb{2}}{3}$}

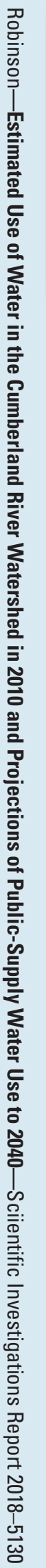

